\title{
A Tutorial on Ultra-Reliable and Low-Latency Communications in 6G: Integrating Domain Knowledge into Deep Learning
}

\author{
Changyang She, Member, IEEE, Chengjian Sun, Student Member, IEEE, Zhouyou Gu, Yonghui Li, Fellow, IEEE, \\ Chenyang Yang, Senior Member, IEEE, H. Vincent Poor Fellow, IEEE, and Branka Vucetic Fellow, IEEE
}

\begin{abstract}
As one of the key communication scenarios in the 5th and also the 6th generation (6G) of mobile communication networks, ultra-reliable and low-latency communications (URLLC) will be central for the development of various emerging mission-critical applications. State-of-the-art mobile communication systems do not fulfill the end-to-end delay and overall reliability requirements of URLLC. In particular, a holistic framework that takes into account latency, reliability, availability, scalability, and decision making under uncertainty is lacking. Driven by recent breakthroughs in deep neural networks, deep learning algorithms have been considered as promising ways of developing enabling technologies for URLLC in future 6G networks. This tutorial illustrates how domain knowledge (models, analytical tools, and optimization frameworks) of communications and networking can be integrated into different kinds of deep learning algorithms for URLLC. We first provide some background of URLLC and review promising network architectures and deep learning frameworks for 6G. To better illustrate how to improve learning algorithms with domain knowledge, we revisit model-based analytical tools and cross-layer optimization frameworks for URLLC. Following that, we examine the potential of applying supervised/unsupervised deep learning and deep reinforcement learning in URLLC and summarize related open problems. Finally, we provide simulation and experimental results to validate the effectiveness of different learning algorithms and discuss future directions.
\end{abstract}

Index Terms-Ultra-reliable and low-latency communications, 6G, cross-layer optimization, supervised deep learning, unsupervised deep learning, deep reinforcement learning.

\section{INTRODUCTION}

As one of the new communication scenarios in the 5th Generation (5G) mobile communications, Ultra-Reliable and LowLatency Communications (URLLC) are crucial for enabling a wide range of emerging applications [1], including industry automation, intelligent transportation, telemedicine, Tactile Internet, and Virtual/Augmented Reality (VR/AR) [2-6]. Since these applications fall within the scope of Industrial Internetof-Things (IoT) [7], Germany Industrie 4.0 [8], and Made in China 2025 [9], URLLC has the potential to improve our

C. She, Z. Gu, Y. Li, and B. Vucetic are with the School of Electrical and Information Engineering, University of Sydney, Sydney, NSW 2006, Australia (e-mail: shechangyang@ gmail.com, \{zhouyou.gu, yonghui.li, branka.vucetic\}@sydney.edu.au).

C. Sun and C. Yang are with the School of Electronics and Information Engineering, Beihang University, Beijing 100191, China (email:\{sunchengjian,cyyang\}@buaa.edu.cn).

H. V. Poor is with the Department of Electrical Engineering at Princeton University, Princeton, NJ 08544, USA. (e-mail: poor@princeton.edu).

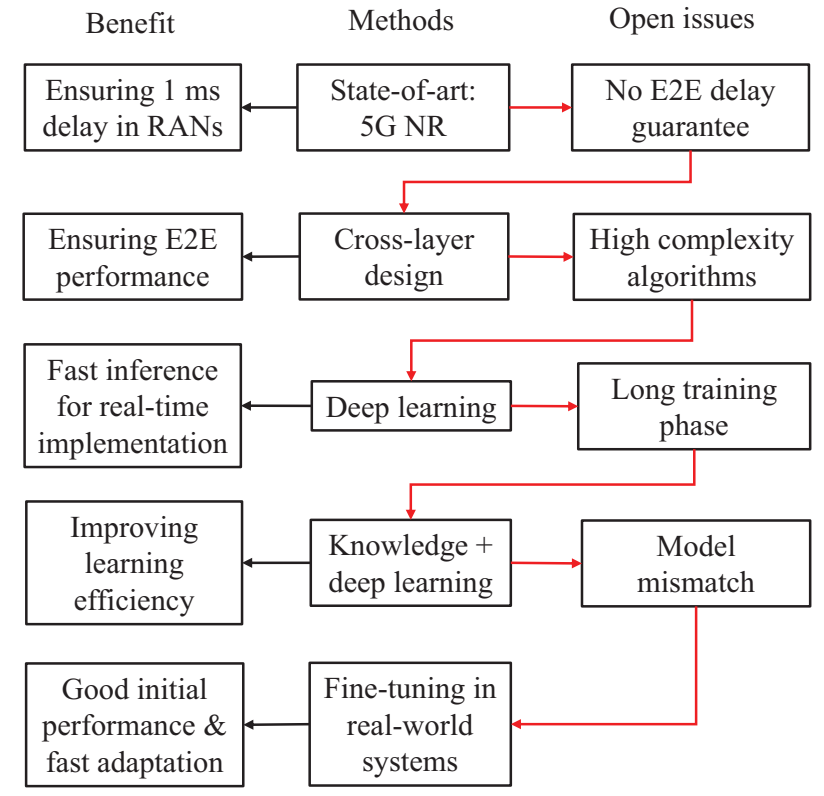

Fig. 1. Potential methods to achieve URLLC [10,12].

everyday life and spur significant economic growth in the future.

\section{A. Motivation}

According to the requirements in $5 \mathrm{G}$ standards [1], to support emerging mission-critical applications, the End-to-End (E2E) delay cannot exceed $1 \mathrm{~ms}$ and the packet loss probability should be $10^{-5} \sim 10^{-7}$. Compared with the existing cellular networks, the delay and reliability require significant improvements by at least two orders of magnitude for $5 \mathrm{G}$ networks. This capability gap cannot be fully resolved by the 5G New Radio (NR), i.e., the physical-layer technology for 5G [13], even though the transmission delay in Radio Access Networks (RANs) achieves the $1 \mathrm{~ms}$ target. Transmission delay contributes only a small fraction of the E2E delay, as the stochastic delays in upper networking layers, such as queuing delay, processing delay, and access delay, are key bottlenecks for achieving URLLC. Beyond 5G systems or socalled Sixth Generation (6G) systems should guarantee the E2E delay bound with high reliability. These stringent requirements create unprecedented research challenges to wireless network design. The open issues and potential methods for 
addressing them are illustrated in Fig. 11 In the sequel, we describe the motivation for applying these methods in URLLC.

1) Cross-layer Design: Existing design methods dividing divide communication networks into multiple layers according to the Open Systems Interconnection model [14]. Communication technologies in each layer are often developed without considering the impacts on other layers, despite the fact that the interactions across different layers are known to significantly impact on the E2E delay and reliability. Most existing approaches do not reflect such interactions; this leads to suboptimal solutions and thus we are yet to be able to meet the stringent requirements of URLLC. To guarantee the E2E delay and the reliability of the communication system, we need accurate and analytically tractable cross-layer models to reflect the interactions across different layers.

2) Deep Learning: With $5 \mathrm{G} \mathrm{NR}$, the radio resources are allocated in each Transmission Time Interval (TTI) with duration of $0.125 \sim 1 \mathrm{~ms}$ [13]. To implement optimization algorithms in $5 \mathrm{G}$ systems, the processing delay should be less than the duration of one TTI. Since the cross-layer models are complex, related optimization problems are non-convex in general (See some examples on cross-layer optimization in [15-19].). The optimization algorithms in these papers require high computing overhead, and hence can hardly be implemented in $5 \mathrm{G}$ systems.

According to several survey papers [20-22], deep learning has significant potential to address the above issue in beyond $5 \mathrm{G} / 6 \mathrm{G}$ networks. The basic idea is to approximate the optimal policy with a deep neural network (DNN). After the training phase, a near-optimal solution of an optimization problem can be obtained from the output of the DNN in each TTI. Essentially, by using deep learning, we are trading off the online processing time with the computing resource for offline training.

3) Integrating Knowledge into Learning Algorithms: Although deep learning algorithms have shown significant potential, the application of deep learning in URLLC is not straightforward. As shown in [10], deep learning algorithms converge slowly in the training phase and need a large number of training samples to evaluate or improve the E2E delay and reliability. If some knowledge of the environment is available, such as the estimated packet loss probability of a certain decision, the system can exploit this knowledge to improve the learning efficiency [23]. Domain knowledge of communications and networking including models, analytical tools, and optimization frameworks have been extensively studied in the existing literature [24,25]. How to exploit them to improve deep learning algorithms for URLLC has drawn significant attention as well, including in [21, 26, 27].

4) Fine-tuning in Real-world Systems: Communication environments in wireless networks are non-stationary in general. Theoretical models used in off-line training may not match this non-stationary nature of practical networks. As a result, a DNN trained off-line cannot guarantee the Quality-of-Service (QoS) constraints of URLLC. Such an issue is referred to as the model-mismatch problem in [12]. To handle the model mismatch, wireless networks should be intelligent to adjust

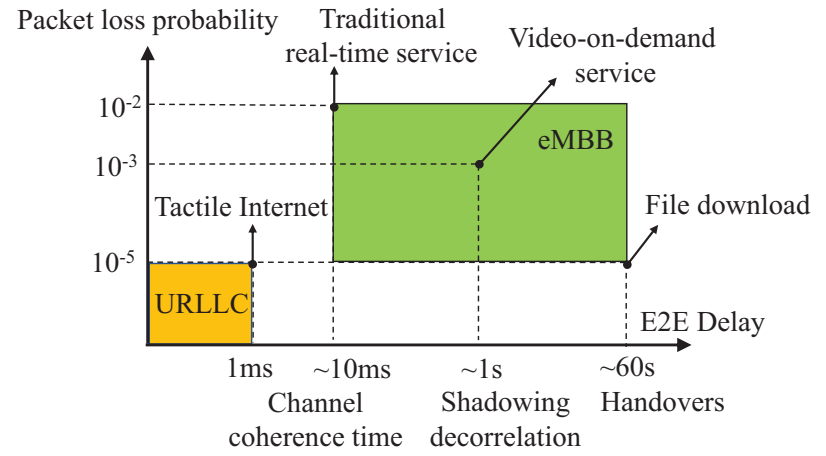

Fig. 2. Delay and reliability requirements of URLLC and eMBB [24 28 29].

themselves in dynamic environments, explore unknown optimal policies, and transfer knowledge to practical networks.

\section{B. Scope of This Paper}

This paper provides a review of domain knowledge (i.e., analytical tools and optimization frameworks) and deep learning algorithms (i.e., supervised, unsupervised, and reinforcement learning). Then, we illustrate how to combine them to optimize URLLC in a cross-layer manner. The scope of this paper is summarized as follows,

1) In Section II, we introduce the background of URLLC including research challenges, methodologies, and a road-map toward URLLC.

2) In Section III, we review promising network architectures and deep learning frameworks for URLLC and summarize the design principles of deep learning frameworks in $6 \mathrm{G}$ networks.

3) We revisit analytical tools in information theory, queuing theory, and communication theory in Section IV, and show how to apply them in cross-layer optimization for URLLC in Section V.

4) Then, we examine the potential of applying supervised/unsupervised deep learning and Deep Reinforcement Learning (DRL) in URLLC in Sections VI, VII, and VIII, respectively. Related open problems are summarized in each section.

5) In Section IX, we provide some simulation and experimental results to illustrate how to integrate domain knowledge into deep learning algorithms for URLLC.

6) Finally, we highlight some future directions in Section $\mathrm{X}$ and conclude this paper in Section XI.

\section{BACKGROUND OF URLLC}

The requirements on E2E delay and overall packet loss probability of URLLC and enhanced Mobile BroadBand (eMBB) services are illustrated in Fig. 2. For eMBB services, communication systems can trade reliability with delay by retransmissions. For URLLC services, the requirements on the E2E delay and reliability are much more stringent than eMBB services. In addition, the wireless channel fading, shadowing, and handovers will have significant impacts on the reliability of URLLC services (See some existing results in [30-32].). Therefore, ensuring the QoS requirements of URLLC is more challenging than eMBB services. 
TABLE I

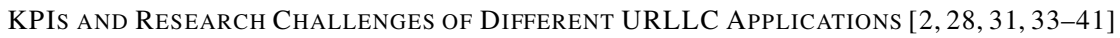

\begin{tabular}{|c|c|c|}
\hline \multicolumn{3}{|c|}{ Indoor large-scale scenarios } \\
\hline Applications & KPIs (except E2E delay \& reliability) & Research Challenges \\
\hline Factory automation & SE, EE \& AoI & Scalability \& network congestions \\
\hline VR/AR applications & SE \& throughput & Processing/transmission 3D videos \\
\hline \multicolumn{3}{|c|}{ Indoor wide-area scenarios } \\
\hline Applications & KPIs (except E2E delay \& reliability) & Research Challenges \\
\hline Tele-surgery & Round-trip delay, throughput \& jitter & Propagation delay \& high data rate \\
\hline eHealth monitoring & EE \& network availability & Propagation delay \& localization \\
\hline \multicolumn{3}{|c|}{ Outdoor large-scale scenarios } \\
\hline Applications & KPIs (except E2E delay \& reliability) & Research Challenges \\
\hline Vehicle safety & AoI, SE, security \& network availability & High mobility, scalability \& network congestions \\
\hline \multicolumn{3}{|c|}{ Outdoor wide-area scenarios } \\
\hline Applications & KPIs (except E2E delay \& reliability) & REesearch Challenges \\
\hline Smart grid & SE, security, network availability \& jitter & Propagation delay \& high data rate \\
\hline Tele-robotic control & EE, security, network availability \& AoI & Propagation delay \& high mobility \\
\hline UAV control & S &
\end{tabular}

\section{A. Other Key Performance Indicators (KPIs) and Challenges}

In the existing literature, the application scenarios, KPIs, and research challenges of URLLC have been extensively investigated. In Table I, we classify these applications into four categories according to the communication scenarios, including indoor large-scale networks, indoor wide-area networks, outdoor large-scale networks, and outdoor wide-area networks.

1) Indoor Large-scale Networks: Typical applications in indoor large-scale networks include factory automation and VR/AR applications such as immersed VR games [6, 8, 34]. As the density of devices increases, improving the spectrum efficiency, say the number of services that can be supported with a given total bandwidth, becomes an urgent task. Since the battery capacities of mobile devices are limited, energy efficiency is another KPI in this scenario. In factory automation, a large number of sensors keep updating their status to the controller and actuator [41], where the freshness of information is one of the KPIs measured by Age of Information (AoI) [42].

In wireless communications, the numbers of constraints and optimization variables increase with the number of devices. Since most of the optimization algorithms in [43] only work well for small and medium scale problems, as the density of devices grows, scalability becomes the most challenging issue in indoor large-scale networks. In addition, stochastic packet arrival processes from a large number of users will lead to strong interference in the air interface. The burstiness and correlation of packet arrivals will result in severe network congestions in queuing systems and computing systems. How to alleviate network congestions in large-scale networks remains an open problem.

2) Indoor Wide-area Networks: For long-distance applications in Tactile Internet, like telesurgery and remote training, the system stability and teleoperation quality are very sensitive to round-trip delay [3744]. Thus, the round-trip delay is a KPI in bilateral teleoperation systems [6]. On the other hand, to maintain stability and transparency, the signals from a haptic sensor are sampled, packetized, and transmitted at $1000 \mathrm{~Hz}$ or even higher [6]. As a result, the required throughput is very high. Meanwhile, the jitter of delay is crucial for the stability of control systems as stated in [33]. If the jitter is larger than the inter-arrival time between two packets (less than $1 \mathrm{~ms}$ in telesurgery), the order of the control commands arriving at the receiver can be different from the order of commands generated by the controller. In this case, the control system is unstable.

Due to long communication distance, the propagation delay in core networks might be much longer than the required E2E delay, and thus will become the bottleneck of URLLC. To handle this issue, prediction and communication co-design is a promising solution, which, however, will introduce extra prediction errors [45]. To guarantee the round-trip delay, overall reliability, and throughput, we need to analyze the fundamental tradeoffs among them and design practical solutions that can approach the performance limit [46].

3) Outdoor Large-scale Networks: One of the most important applications in outdoor large-scale networks is the vehicle safety application [36]. With the current technologies for autonomous vehicles, no information is shared among vehicles. Thus, vehicles are not aware of potential threats from the blind areas of their image-based sensors and distance sensing devices [47]. URLLC can enhance road safety by sharing street maps and safety messages among vehicles. Like indoor large-scale networks, the spectrum efficiency is one of the major KPIs in outdoor large-scale networks with high user density. To maintain current state information from nearby vehicles, minimizing AoI is helpful for improving road safety in vehicular networks [48]. In addition, outdoor wireless networks are more vulnerable than indoor networks. Potential eavesdroppers can receive signals and crack the 
information with high probabilities. Therefore, security should be considered for URLLC in outdoor wireless networks [49].

Current cellular networks can achieve $95 \%$ coverage probability, which is satisfactory for most of the existing services. In URLLC, the required network availability can be up to $99.999 \%$ [50]. As a result, we need to improve the network availability by several orders of magnitude. Due to high mobility, service interruptions caused by frequent handovers and network congestions are the bottlenecks for achieving high availability. Besides, existing tools for analyzing availability are only applicable in small-scale networks [51, 52]. How to analyze and improve network availability in large-scale networks remains unclear.

4) Outdoor Wide-area Networks: Unmanned Aerial Vehicle (UAV) control, telerobotic control, and smart grids are typical applications in outdoor wide-area networks [2, 31, 39]. Establishing secure, reliable, and real-time control and non-payload communication (CNPC) links between UAVs and ground control stations/devices or satellites in a wide-area has been considered as one of the major goals in future space-air-ground integrated networks [53]. Thus, the KPIs in outdoor widearea networks include security, network availability, roundtrip delay, AoI and jitter.

In UAV control, there are two ways to maintain CNPC links: ground-to-air communications and satellite communications [54,55]. Nevertheless, ground-to-air links may not be available in rural areas, where the density of ground control stations is low. If packets are sent via satellites, they suffer from long propagation delays and coding delays. In smart grids, although the E2E delay and overall reliability are less stringent than factory automation, the long communication distance leads to long propagation delay [2]. As a result, achieving the target KPIs in outdoor wide-area networks is still very challenging with current communication technologies.

\section{B. Tools and Methodologies for URLLC}

To achieve the target KPIs, we need to revisit analytical tools and design methodologies in wireless networks.

1) Analytical Tools: To analyze the performance of a wireless network, a variety of theoretical tools have been developed in the existing literature [24, 25]. For example, to reduce transmission delay, several kinds of channel coding schemes with short blocklength have been developed in the existing literature [28]. To obtain the achievable rate in the short blocklength regime, a new approximation was derived by Y. Polyanskiy et al. [56]. It indicates that the decoding error probability does not vanish for arbitrarily finite Signalto-Noise Ratio (SNR) when the blocklength is short. Such an approximation can characterize the relationship between data rate and decoding error probability, and is fundamentally different from Shannon's capacity that was widely used to design wireless networks.

If we can derive the closed-form expressions of KPIs in Table I, then it is possible to predict the performance of a solution or decision. The disadvantage is that theoretical analytical tools are based on some assumptions and simplified models that may not be accurate enough for URLLC applications.
Moreover, to analyze the E2E performance in URLLC, the models may be very complicated, and closed-form results may not be available.

Another approach is to evaluate the performance of the solution with simulation or experiment. The advantage is that they do not rely on any theoretical assumptions. On the negative side, the evaluation procedure is time-consuming, especially for URLLC. For example, to validate the packet loss probability (around $10^{-5} \sim 10^{-7}$ in URLLC) of a user, the system needs to send $10^{8} \sim 10^{10}$ packets.

2) Cross-layer Optimization: The E2E delay and the overall packet loss probability depend on the solutions in different layers [14]. To optimize the performance of the whole system, we need cross-layer optimization frameworks, from which it is possible to reveal some new technical issues by analyzing the interactions among different layers. For example, as shown in [57], when the required queuing delay is shorter than the channel coherence time, the power allocation policy is a type of channel inverse policy, which can be unbounded over typical wireless channels, such as the Nakagami- $m$ fading channel. One way to analyze the reliability of a wireless link is to analyze the outage probability [58]. However, such a performance metric cannot characterize the queuing delay violation probability in the link layer. To keep the decoding error probability and the queuing delay violation probability below the required thresholds, some packets should be dropped proactively when the channel is in deep fading [16]. By cross-layer design, one can take the delay components and packet losses in different layers into account, and hence it is possible to achieve the target E2E delay and overall packet loss probability in URLLC.

Although cross-layer optimization has the potential to achieve better E2E performances than dividing communication systems into separated layers, to implement cross-layer optimization algorithms in practical systems, one should address the following issues,

- High computing overheads: Wireless networks are highly dynamic due to channel fading and traffic load fluctuations. As a result, the system needs to adjust resource allocation frequently according to these time-varying factors. Without the closed-form expression of the optimal policy, the system needs to solve the optimization problem in each TTI. This will bring very high computing overheads. Even if the problem is convex and can be solved by existing searching algorithms, such as the interior-point method [43], the algorithms are still too complicated to be implemented in real-time.

- Intractable problems: Owning to complicated models from different layers, cross-layer optimization problems are usually non-convex or Non-deterministic Polynomialtime (NP)-hard. Solving NP-hard optimization problems usually takes a long time, and the resulting optimization algorithms can hardly be implemented in real-time. Lowcomplexity numerical algorithms that can be executed in practical systems for URLLC are still missing.

- Model mismatch: Since the available models in different layers are not exactly the same as practical systems, the model mismatch may lead to severe QoS violations in 
real-world networks. How to implement the solutions and policies obtained from the analysis and optimization in practical systems remains an open problem.

3) Deep Learning for URLLC: Unlike optimization algorithms, deep learning approaches can be model-based or model-free [27, 59, 60], and have the potential to be implemented in real-world communication systems [61]. The advantages of deep learning algorithms can be summarized as follows.

- Real-time implementation: After off-line training, a DNN serves as a mapping from the observed state to a nearoptimal action in communication systems, where the forward propagation algorithm is used to compute the output of a given input. According to the analysis in [12], the computational complexity of the forward propagation algorithm is much lower than searching algorithms for cross-layer optimizations. Therefore, by using DNN in $5 \mathrm{G} \mathrm{NR}$, it is possible to obtain a near-optimal action within each TTI.

- Searching optimal policies numerically: When optimal solutions are not available, there is no labeled sample for supervised learning. To handle this issue, the authors of [26, 62, 63] proposed to use unsupervised deep learning algorithms to search the optimal policy. Unlike optimization algorithms that find an optimal solution for a given state of the system, unsupervised deep learning algorithms find the form of the optimal policy numerically. The basic idea is to approximate the optimal policy with a DNN and optimize the parameters of the DNN towards a loss function reflecting the design goal.

- Exploring optimal policies in real-world networks: When there is no labeled sample or theoretical model to formulate an optimization problem, we can use DRL to explore optimal policies from the real-world network [60]. For example, an actor-critic algorithm uses two DNNs to approximate the optimal policy and value function, respectively. From the feedback of the network, the two DNNs are updated until convergence [64].

Although deep learning algorithms have the above advantages, to apply them in URLLC, the following issues remain unclear.

- QoS guarantee: When designing wireless networks for URLLC, the stringent QoS requirements should be satisfied. When using a DNN to approximate the optimal policy, the approximation should be accurate enough to guarantee the QoS constraints. Although the universal approximation theorem of DNNs indicates that a DNN can be arbitrarily accurate when approximating a continuous function [65, 66], how to design structures and hyperparameters of DNNs to achieve a satisfactory accuracy remains unclear.

- Learning in non-stationary networks: When applying supervised deep learning in URLLC, the DNN is trained off-line with a large number of training samples [11]. When using unsupervised deep learning to find the optimal policy, we need to formulate an optimization problem by assuming the system is stationary [67]. Both approaches can perform very well in stationary networks [11, 67]. However, when the environment changes, the DNN trained off-line can no longer guarantee the QoS constraints of URLLC. To handle this issue, the system needs to adjust the DNN in non-stationary environments with few training samples [12].

- Exploration safety of DRL: To improve the policy in the unknown environment, a DRL algorithm will try some actions randomly to estimate the reward of different actions [68]. During explorations, the DRL algorithm may try some bad actions, which will deteriorate the QoS significantly and may lead to unexpected accidents in URLLC systems. Thus, the exploration safety will become a bottleneck for applying DRL in URLLC.

\section{Road-map Toward URLLC in $6 G$ Networks}

Based on the KPIs and challenges of different applications in Section $\Pi-\mathrm{A}$ and the tools and methodologies in Section II-B, the road-map toward URLLC in 6G networks is summarized in Fig. 3. As indicated in [10], by integrating domain knowledge into deep learning algorithms, it is possible to provide in-depth understandings and practical solutions for URLLC.

Step 1 (Knowledge-based analysis and optimization): To satisfy the QoS requirements of URLLC in real-world systems, one should first take the advantage of the knowledge in information theory, queuing theory, and communication theory [69]. From theoretical analytical tools and cross-layer optimization frameworks, we can obtain the performance limits on the tradeoffs among different KPIs [16, 56, 70].

Step 2 (Knowledge-assisted training of deep learning): Based on analytical tools and optimization frameworks in communications and networking, one can build a simulation platform to train deep learning algorithms [27,59]. In supervised deep learning, we can find labeled training samples from the simulation platform [11]. In unsupervised deep learning, the knowledge of the communication systems can be used to formulate the loss function [67]. In DRL, by initializing algorithms off-line, the exploration safety and convergence time of DRL algorithms can be improved significantly [71].

Step 3 (Fine-tuning deep learning in real-world networks) From the previous step, DNNs are pre-trained with the help of models, analytical tools, and optimization frameworks that capture some key features of real-world systems. Since the models are not exactly the same as the real-world systems, we need to fine-tune DNNs in non-stationary networks. As shown in [60], data-driven deep learning updates the initialized DNNs by exploiting real-world data and feedback. After finetuning, we can obtain practical solutions from the outputs of the DNNs in real-time.

\section{Related Works}

1) Survey and Tutorial of URLLC: URLLC was included in the specification of $3 \mathrm{GPP}$ in 2016. Since then, great efforts have been devoted to this area [14,72,-77]. The review from the information theory aspect was published in 2016 [72], where the analyses of the impacts of short blocklength channel 


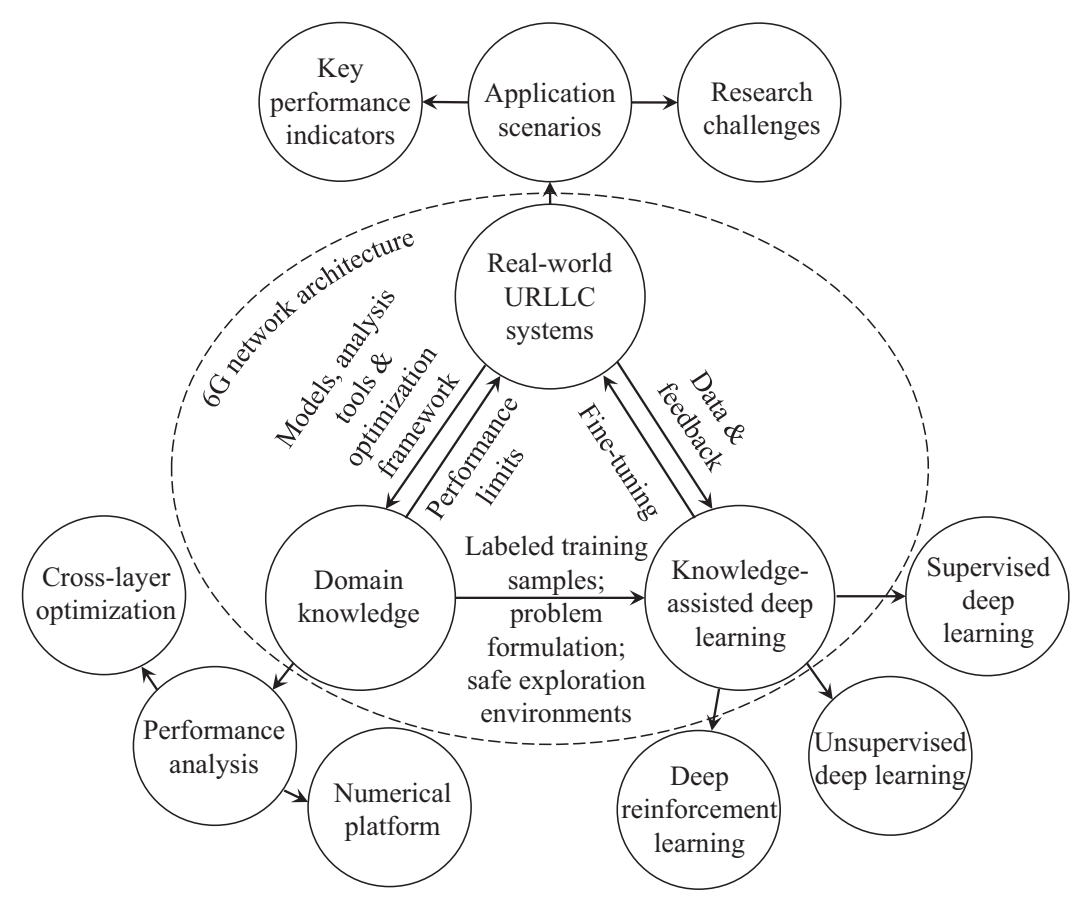

Fig. 3. Road map toward URLLC in 6G networks [10 11].

codes on the latency and reliability were discussed. With a focus on standard activities, A. Nasrallah et al. [74] provided a comprehensive overview of the IEEE 802.1 Time-Sensitive Networking (TSN) standard and Internet Engineering Task Force Deterministic Networking standards as the key solutions in the link and network layers, respectively. X. Jiang et al. [14] proposed a holistic analytical and classification of the main design principles and technologies that will enable low-latency wireless networks, where the technologies from different layers and cross-layer design were summarized. With the focus on the physical layer, K. S. Kim introduced solutions for spectrally efficient URLLC techniques [75]. G. Sutton et al. reviewed the technologies for URLLC in the physical layer and the medium access control layer technologies [78]. K. Antonakoglou et al. [73] introduced haptic communication as the third media stream that will complement audio and vision over the Internet. The evaluation methodologies and necessary infrastructures for haptic communications were discussed in [73]. I. Parvez et al. [76] presented a general view on how to meet latency and other $5 \mathrm{G}$ requirements with Software-Defined Network (SDN), network function virtualization, caching, and Mobile Edge Computing (MEC). M. Bennis et al. noticed that the existing design approaches mainly focus on average performance metrics and hence are not applicable in URLLC (e.g., average throughput, average delay, and average response time) [77]. They summarized the tools and methodologies to characterize the tail distribution of the delay, service quality, and network scalability.

2) Survey and Tutorial of Deep Learning in Wireless Networks: Deep learning has been considered as one of the key enabling technologies in the intelligent 5G [79] and the beyond $5 \mathrm{G}$ cellular networks [80,81]. To combine deep learning with wireless networks, several comprehensive surveys and reviews have been carried out [20, 21, 60, 82,-86]. J. Wang et al. presented a thorough survey of machine learning algorithms and their use in the next-generation wireless networks in [82]. Q. Mao et al. [83] reviewed how to use deep learning in different layers of the OSI model and cross-layer design. With the focus on the smart radio environment, A. Zappone et al. proposed to integrate deep learning with traditional model-based technologies in future wireless networks [21]. A comprehensive survey of the crossovers between deep learning and wireless networks was presented by C. Zhang et al. in [20], where the authors illustrated how to tailor deep learning to mobile environments. N. C. Luong et al. presented a tutorial on DRL and reviewed the applications of DRL in wireless communications and networking [60]. A tutorial on how to use deep/reinforcement learning for wireless resource allocation in vehicular networks was presented by L. Liang et al. in [84]. Y. Sun et al. summarized some key technologies and open issues of applying machine learning in wireless networks in [85]. J. Park et al. provided a tutorial on how to enable wireless network intelligence at the edge [86].

Although the above two branches of existing works either investigated model-based approaches for URLLC or reviewed deep learning in wireless networks. None of them discussed how to optimize wireless networks for URLLC by integrating domain knowledge of communications and networking into deep learning, which is the central goal of our work. Different from existing papers, we revisit the theoretical models, analytical tools, and optimization frameworks, review different deep learning algorithms for URLLC. Furthermore, we illustrate how to combine them together to achieve URLLC. 


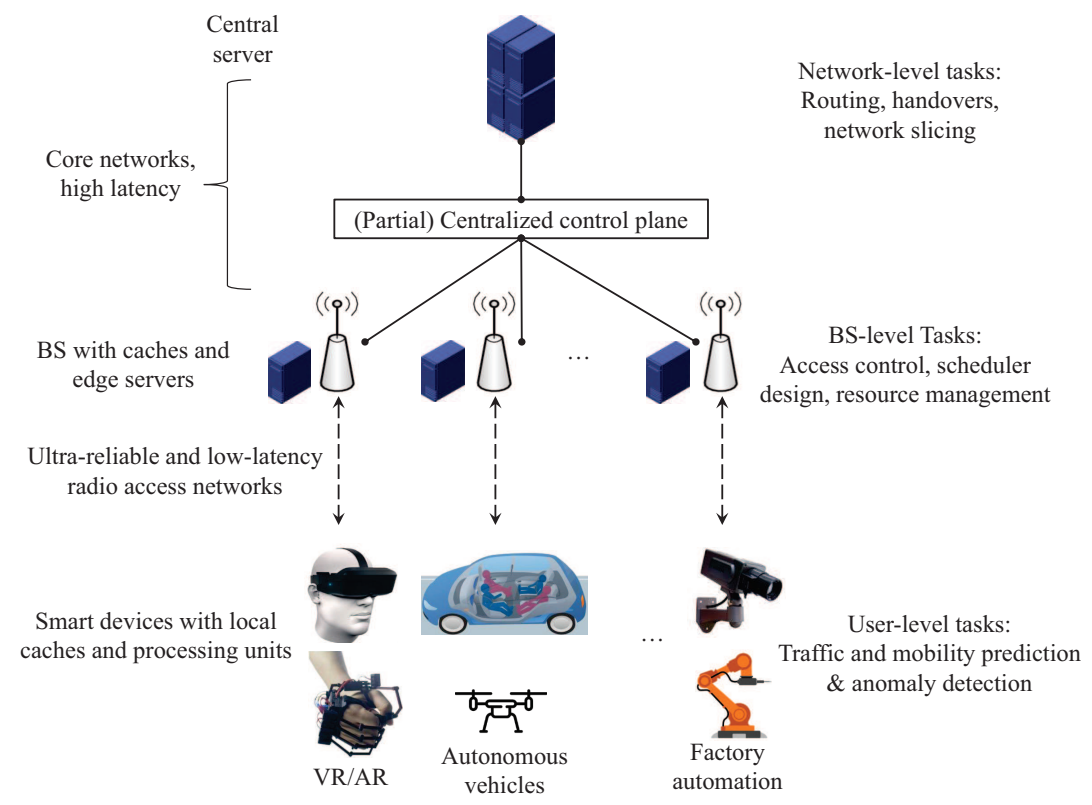

Fig. 4. A general architecture in 6G networks [10 88 89].

\section{Network ARChitectures AND DEEP LEARNING FRAMEWORKS IN 6G}

The application of machine learning in cellular networks has gained tremendous attention in both academia and industry. Recently, how to operate cellular networks with machine learning algorithms had been considered as one of the 3GPP work items in [87]. Since computing and storage resources will be deployed at both edge and central servers of $6 \mathrm{G}$ networks, $6 \mathrm{G}$ will have the capability to train deep learning algorithms [86]. In this section, we review some network architectures and illustrate how to develop deep learning frameworks.

\section{A. Network Architectures}

A general network architecture for various application scenarios (such as IoT networks [88] and vehicular networks [89]) in 6G networks is illustrated in Fig. 4 As shown in [10], the communication, computing, and caching resources are integrated into a multi-level system to support tasks at the user level, the Base Station (BS) level, and the network level. Such an architecture can be considered as a ramification of the following architectures.

1) $M E C:$ As a new paradigm of computing, MEC is believed to be a promising architecture for URLLC [90]. Unlike centralized mobile cloud computing with routing delay and propagation delay in backhauls and core networks, the E2E delay in MEC systems consists of Uplink (UL) and Downlink (DL) transmission delays, queuing delays in the buffers of users and BSs, and the processing delay in the MEC [91]. The results in [91] indicate that it is possible to achieve $1 \mathrm{~ms}$ E2E delay in MEC systems by optimizing task offloading and resource allocation in RANs.

To analyze the E2E delay and overall packet loss probability of URLLC, Y. Hu et al. considered both decoding error probability in the short blocklength regime and the queuing delay violation probability [92]. C.-F. Liu et al. minimized the average power consumption of users subject to the constraint on delay bound violation probability [93]. In multi-cell MEC networks, J. Liu et al. investigated how to improve the tradeoff between latency and reliability [94], where a typical user is considered. To further exploit the computing resources from the central server, a multi-level MEC system was studied in heterogeneous networks, where computing-intensive tasks can be offloaded to the central server [95].

To implement the existing solutions in real-world MEC systems, some problems remain open:

- Optimization complexity: Optimization problems in MEC systems are general non-convex. To find the optimal solution, the computing complexity for executing searching algorithms is too high to be implemented in real-time.

- Mobility of users: In vehicle networks, users are moving with high speed. The key issue here is adjusting task offloading during frequent handovers. How to optimize task offloading for high mobility users remains unclear.

- High overhead for exchanging information: Current task offloading and resource allocation algorithms are executed at the central controller. This will lead to high overhead for exchanging information among each BS and the central controller. To avoid this, we need distributed algorithms for URLLC, which are still unavailable in the existing literature.

- Serving hybrid services: VR/AR applications are expected to provide immersion experience to users [96]. To achieve this goal, the system needs to send $360^{\circ}$ video as well as tactile feedback to each user. How to optimize task offloading for both video and tactile services in VR/AR applications needs further research.

2) Multi-Connectivity and Anticipatory Networking: To achieve a high network availability, an effective approach is to serve each user with multiple links, e.g., multi-connectivity [97]. Such an approach will bring two new research chal- 
lenges: 1) improving the fundamental tradeoff between spectrum efficiency and network availability [98] and 2) providing seamless service for moving users [99].

As illustrated in [51], one way to improve network availability without sacrificing spectrum efficiency is to serve each user with multiple BSs over the same subchannel (or subcarriers). Such an approach is referred to as intra-frequency multiconnectivity. The disadvantage of this intra-frequency multiconnectivity is that the failures of different links are highly correlated. For example, if there is a strong interference on a subchannel, then the signal to interference plus noise ratios of all the links are low. To alleviate cross-correlation among different links, different nodes can connect to one user with different subchannels or even with different communication interfaces [98]. To achieve a better tradeoff between spectrum efficiency and availability, network coding is a viable solution [100]. Most of the existing network coding schemes are too complicated to be implemented in URLLC. To reduce the complexity of signal processing, configurable templates for network coding were developed in [101].

To provide seamless service for moving users, anticipatory networking is a promising solution [102]. The basic idea of anticipatory networking is to predict the mobility of users according to their mobility pattern and reserve resources before handovers proactively. N. Bui et al. validated that anticipatory networking allows network operators to save half of the resources in Long Term Evolution systems [103. More recently, K. Chen et al. proposed a proactive network association scheme, where each user can proactively associate with multiple BSs [99]. As proved in [104], the requirements on queuing delay and queuing delay violation probability can be satisfied with this scheme.

3) SDN and Network Slicing: SDN architectures have been adopted by 5G Infrastructure Public Private Partnership (5G-PPP) architecture working group [105]. By splitting the control plane and the user plane, the controller can manage radio resources and data flows in fully centralized, partially centralized, and fully decentralized manners. Essentially, there is a tradeoff between control-plane overheads and user-plane QoS. To achieve a better tradeoff, 5G-PPP structured network functions into the following three parts according to their position in the protocol stack [105]: high layer, medium layer, and low layer. The high layer control plane manages resource coordination and long-term load balancing for QoS guarantee and network slicing. The medium layer control plane handles mobility and admission control. The low layer control plane deals with short-term scheduling and physicallayer resource management, which requires low control-plane latency and high control-plane reliability. To address these issues, short frame structure and grant-free scheduling have been developed in 5G NR to reduce latency; Polar codes will be applied in control signaling with short blocklength [13]. In addition, IEEE TSN standard in wired communications and other standards on synchronization, cyclic queuing and forwarding, frame preemption, stream reservation have specified the control signaling to achieve low latency, low jitter, and low packet loss probability [106].

Since the control plane is deployed at different layers, there is no need to collect all the network state information at the central controller. Network resources (e.g., computing resources [107], storage resources [108], and physical resource blocks [109]) are allocated to different slices according to the slow varying traffic loads and QoS requirements. To guarantee the QoS of different services, the system needs to map the QoS requirements to network resources [110]. However, the E2E delay and overall packet loss probability depend on physicallayer resource allocation, link-layer transmission protocols and schedulers, as well as network architectures. Thus, how to quantify the network resources required by URLLC remains unclear. Most of the existing papers on network slicing either assumed that the required resources to guarantee the QoS of each service are known at the central controller [108] or considered a simplified model to formulate QoS constraints [111]. To fully address this issue, a cross-layer model for E2E optimization is needed.

\section{B. Deep Learning Frameworks}

To cope with the tasks at different levels in Fig. 4 a deep learning framework should exploit computing and storage resources at different entities [10]. A general deep learning framework is illustrated in Fig. 5, which is built upon the following subframeworks.

1) Federated Learning: In the multi-level computing system in Fig. 4 we can hardly obtain well-trained DNNs with local resources and data since the computing resources and the number of training samples at a user or a BS are not enough. Federated learning is capable to exploit local DNNs trained with local data to learn global DNNs [113]. To obtain a global DNN, the basic idea is to compute the weighted sum of the parameters of local DNNs. In this way, each user or BS only needs to upload its local or edge DNN to the central server, and does not need to share its data. Such a framework can avoid high communication overheads and the security issue caused by collecting private training data from all the users [114].

Federated learning was used to evaluate the trail probability of delay in URLLC [115], where the basic idea is to collect the estimated parameters from all the users and perform a global average. Such an approach highly relies on the assumption that the local training data at different users are Independent and Identically Distributed (IID). To implement federated learning with non-IID data sets, solutions have been proposed in two of the most recent papers [116, 117]. However, both of these works were not focused on URLLC. How to guarantee the QoS requirements of users with non-IID data deserves further research.

2) Deep Transfer Learning: Transfer learning aims to apply knowledge learned in previous tasks to novel tasks [118]. Recently, transfer learning was combined with DNNs, and the combined approach is referred to as deep transfer learning [119]. Different from training a new DNN from scratch, deep transfer learning only needs a few training samples to update the DNN trained for the previous task. Such a feature of deep transfer learning brings several advantages in wireless networks: 


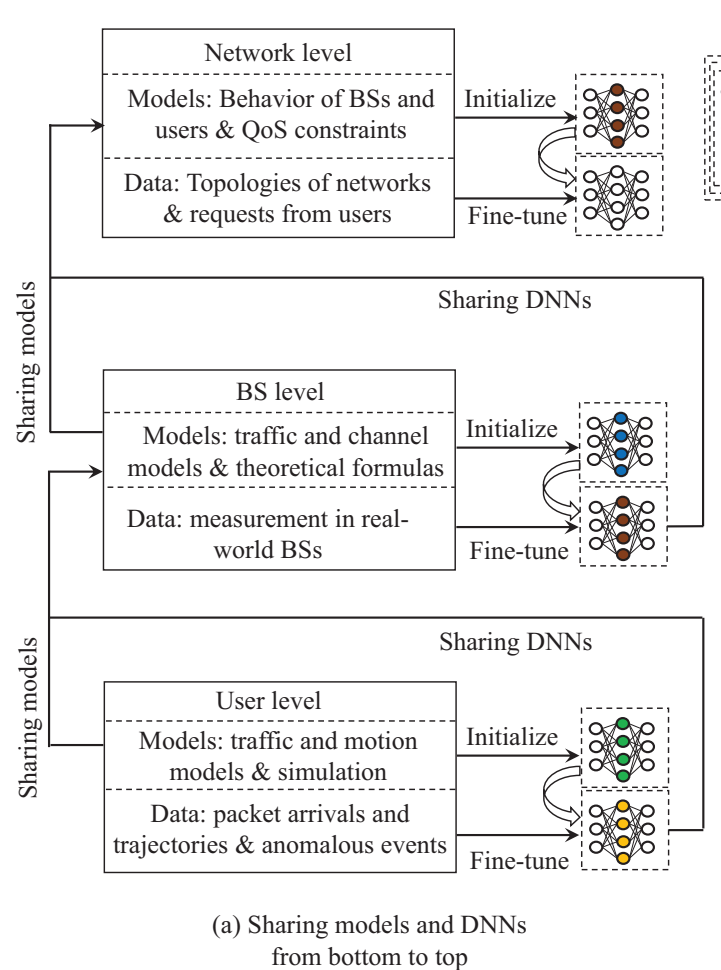

Central library with global DNNs

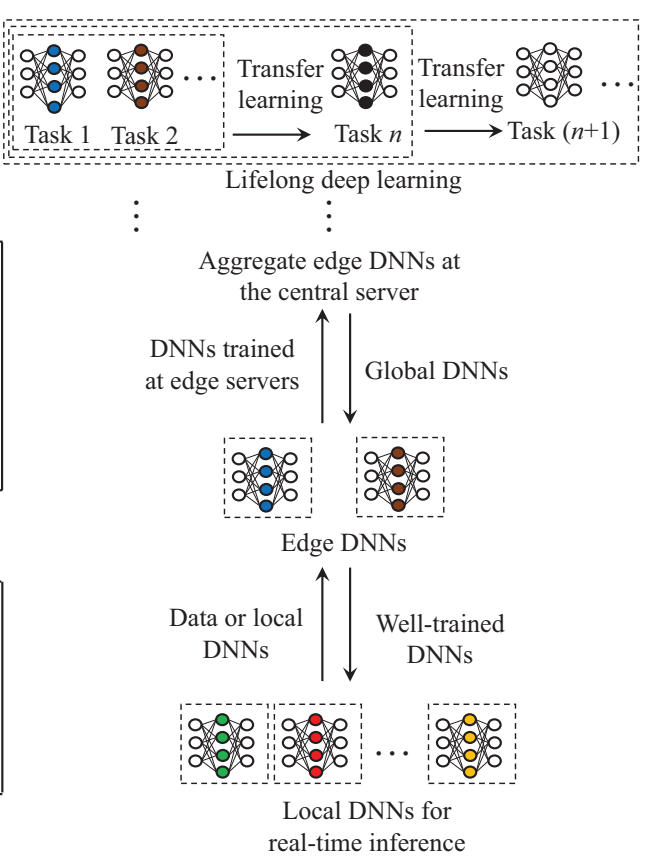

(b) Sharing storage and computing resources from top to bottom

Fig. 5. A general deep learning framework [10 112].

- Handle model mismatch problem: In wireless networks, the models for performance analysis and optimization are not exactly the same as real-world systems. To implement a DNN that is trained from theoretical models, we can use deep transfer learning to fine-tune the DNN [11].

- Deep learning in non-stationary networks: In nonstationary wireless networks, the pre-trained DNN needs to be updated according to the non-stationary environments. Since it is difficult to obtain a large number of new training samples within a short time in the new environment, deep transfer learning should be applied.

- Applying global DNNs at local devices: Considering that the data sets at different devices or BSs follow different distributions [116 117], if a global DNN is directly used by a device or a BS for inference, it can neither guarantee the QoS requirements nor achieve optimal resource utilization efficiency. To avoid this issue, deep transfer learning should be used to fine-tune a global DNN with local training samples.

3) Lifelong Deep Learning: As shown in [120], lifelong learning is a kind of cumulative learning algorithm that consists of multiple steps. In each step, transfer learning is applied to improve the learning efficiency of the new task. As illustrated in Fig. 5, the knowledge learned from the previous $(n-1)$ tasks will be reused to initialize the $n$-th new task. After the training stage, the $n$-th task becomes a source task and will be reused to train new tasks in the future. For example, by combining the federated learning framework with lifelong learning, a lifelong federated reinforcement learning framework for cloud robotic systems was proposed by B. Liu et al. [112]. Such a framework can be adopted in the network architecture in Fig. 4 where the DNNs for different tasks are saved in a central library at the central server. For each device or BS, it fetches the global DNN of the relevant task and finetunes it according to the environment. Finally, a local DNN can be obtained for real-time inference.

4) Distributed Deep Learning: To avoid high overheads for exchanging information, the control plane of a cellular network should not be fully centralized [105]. In other words, each control plane makes its' own decisions according to the available information. To guarantee the QoS requirements of URLLC with a partially centralized or a fully decentralized control plane, a distributed algorithm is needed [121].

In a system, where users and BSs make decisions in a distributed way, the problem can be formulated as a fully cooperative game to improve the global network performance [122]. To find stationary solutions of the game, multi-agent (deep) reinforcement learning turns out to be a promising approach as shown in [123, 124].

\section{Summary of Design Principles}

The principles for designing deep learning frameworks in 6G networks are summarized as follows.

1) Sharing Models and DNNs from Bottom to Top: As illustrated in Fig. 5 a), the tasks in the network level and the BS level depend on the behaviors of BSs and users, respectively. When optimizing policies in the higher levels, the system needs to acquire the models at lower levels [11]. Alternatively, the system can share DNNs that mimic the behaviors of BSs and users with the centralized control plane. For example, when the theoretical models are inaccurate and the number of real-world data samples is limited, Generative Adversarial 
Networks (GANs) can be used to approximate the distributions of packet arrival processes or wireless channels [125]. When the generator networks are available at the centralized control plane, there is no need to share traffic and channel models.

2) Sharing Storage and Computing Resources from Top to Bottom: To guarantee the data-plane latency, the control plane needs to make decisions according to dynamic traffic states and channel states in real-time [105]. With well-trained DNNs, it is possible for BSs and mobile devices to obtain inference results within a short time. However, the training phase of a deep learning algorithm usually needs huge storage and computing resources. As a result, a BS or a device can hardly obtain a well-trained DNN with local training samples and computing resources. As shown in Fig. 5(b), to enable deep learning at lower levels, the central server needs to share storage and computing resources to BSs and devices by federated learning.

3) Tradeoffs among Communications, Computing, and Caching: Sharing information among different levels helps saving computing and caching resources at edge servers and mobile devices at the cost of introducing extra communication overheads. Meanwhile, communication delay and reliability affect the performance of learning algorithms [126127]. Thus, when developing a deep learning framework in a wireless network, the tradeoffs among communications, computing, and caching should be examined carefully. Novel approaches that can improve these tradeoffs are in urgent need [114,127].

\section{Tools for Performance Analysis in URLLC}

To illustrate how to improve deep learning with theoretical tools and models, we revisit tools in information theory, queuing theory, and communication theory for performance analysis in URLLC.

\section{A. Information Theory Tools}

The fundamental relationship between the achievable rate and radio resources is crucial for formulating optimization problems in communication systems. In 1948, the maximal error-free data rate that can be achieved in a communication system was derived in [128], which is known as Shannon's capacity. For example, over an Additive White Gaussian Noise (AWGN) channel, Shannon's capacity can be expressed as follows,

$$
R=W \log _{2}(1+\gamma) \text { (bits/s), }
$$

where $W$ is the bandwidth of the channel and $\gamma$ is the SNR. In the past decades, researchers tried to develop a variety of coding schemes to approach the performance limit. Meanwhile, Shannon's capacity was widely applied in communication system design.

It is well known that Shannon's capacity can be approached when the blocklength of channel codes goes to infinity. However, to avoid a long transmission delay in URLLC, the blocklength should be short. As a result, Shannon's capacity cannot be achieved, and the decoding error probability is nonzero for arbitrary high SNRs.

There are two branches of research in the finite blocklength regime. The first one is analyzing the delay (i.e., coding blocklength) and reliability tradeoffs that can be achieved with different coding schemes [129-136]. For example, a lower bound on the bit error rate of finite-length turbo codes was derived in [129]. The performance of finite-length Low-Density Parity-Check codes was analyzed in [130]. The authors of [132] investigated how to adjust the blocklength of polar codes to keep the BLock Error Rate (BLER) constant.

The second branch of research aims to derive the performance limit on the achievable rate in the short blocklength regime [56, 70, 72, 137,-139]. The milestones of the achievable rate in the short blocklength regime are summarized in Table III

TABLE II

Achievable Rate in the Short Blocklength Regime

\begin{tabular}{|r|l|l|}
\hline Year & Reference & Channel Model \\
\hline 2010 & {$[56]$} & AWGN channel \\
\hline 2011 & {$[140]$} & Gilbert-Elliott Channel \\
\hline 2011 & {$[137]$} & Scalar coherent fading channel \\
\hline 2014 & {$[70]$} & $\begin{array}{l}\text { Quasi-static Multiple-Input-Multiple- } \\
\text { Output (MIMO) channel }\end{array}$ \\
\hline 2014 & {$[138]$} & $\begin{array}{l}\text { Coherent Multiple-Input-Single-Output } \\
\text { (MISO) channel }\end{array}$ \\
\hline 2016 & {$[139]$} & Multi-antenna Rayleigh fading channel \\
\hline 2019 & {$[141]$} & $\begin{array}{l}\text { Coherent multiple-antenna block- } \\
\text { fading channels }\end{array}$ \\
\hline 2019 & {$[142]$} & $\begin{array}{l}\text { Saddlepoint Approximations, Rayleigh } \\
\text { block-fading channels }\end{array}$ \\
\hline
\end{tabular}

To show the difference between the achievable rate in the short blocklength regime and Shannon's capacity, we rephrase some results in [56] here. The achievable rate in short blocklength regime over an AWGN channel can be accurately approximated by the normal approximation, i.e.,

$$
R \approx \frac{W}{\ln 2}\left[\ln (1+\gamma)-\sqrt{\frac{V}{L_{\mathrm{B}}}} f_{\mathrm{Q}}^{-1}\left(\varepsilon_{\mathrm{c}}\right)\right] \quad(\text { bits } / \mathrm{s}),
$$

where $L_{\mathrm{B}}$ is the blocklength, $\varepsilon_{\mathrm{c}}$ is the decoding error probability, $f_{\mathrm{Q}}^{-1}(\cdot)$ is the inverse of Q-function, and $V$ is the channel dispersion, which can be expressed as $V=1-\frac{1}{(1+\gamma)^{2}}$ over the AWGN channel. The blocklength $L_{\mathrm{B}}$ is the number of symbols in each block. To transmit $L_{\mathrm{B}}$ symbols, the amount of time and frequency resources can be obtained from $D_{\mathrm{t}} W=L_{\mathrm{B}}$, where $D_{\mathrm{t}}$ is the transmission duration of the $L_{\mathrm{B}}$ symbols.

The difference between (1) and (2) lies in the second term in (2). When the blocklength $L_{\mathrm{B}}$ goes to infinite, then the achievable rate in (2) approaches Shannon's capacity. To transmit $b$ bits in one block with duration $D_{\mathrm{t}}$, the decoding error probability can be derived as follows,

$$
\varepsilon_{\mathrm{c}} \approx f_{\mathrm{Q}}\left(\sqrt{\frac{D_{\mathrm{t}} W}{V}}\left[\ln (1+\gamma)-\frac{b \ln 2}{D_{\mathrm{t}} W}\right]\right),
$$

which is obtained by substituting (2) into $D_{\mathrm{t}} R=b$. From (3) we can see that to keep the decoding error probability constant, the required bandwidth decreases with the transmission duration. Essentially, there are tradeoffs among the 
transmission delay, the spectrum efficiency, and the decoding error probability.

Considering that the result in (3) is an approximation that cannot guarantee the required decoding error probability, the lower and upper bounds of the achievable rate were provided in [142]. The authors further illustrated that the Saddlepoint Approximation derived in this paper lies between the lower and upper bounds, and is more accurate than the normal approximation over Rayleigh fading channel. However, Saddlepoint Approximation has no closed-form expression in general, and hence we cannot obtain closed-form expressions of QoS constraints by using Saddlepoint Approximation. As shown in [28], when the BLER is $10^{-7}$, the gap between the normal approximation and practical channel coding schemes, such as the extended Bose, Chaudhuri, and Hocquenghem $(\mathrm{eBCH})$ code, is less than $0.1 \mathrm{~dB}$. Thus, (2) and (3) are still good choices in practical system design.

\section{B. Queuing Theory Tools}

Depending on the delay metrics, the existing queuing theory research papers can be classified into three categories, i.e., average delay [143- -146$]$, hard deadline [15, 147- 150], and statistical QoS [16, 151-157].

1) Average Queuing Delay: Let us consider a queuing system with average packet arrival process $\bar{a}(t)$, where $a(t)$ is the data arrival rate at time $t$. Then, the average queuing delay $\bar{D}(t)$ and the average queue length $\bar{Q}(t)$ satisfy a simple and exact relation [25], i.e.,

$$
\bar{Q}(t)=\bar{D}(t) \bar{a}(t)
$$

which is the famous Little's Law. It is a very general conclusion that does not depend on the service order of the queuing system, the length of the buffer, or the distributions of the inter-arrival time between packets and the service time of each packet. The only assumption is that the arrival processes and service processes are stationary.

The average delay metric is suitable for services with no strict delay requirement, such as file download. However, it is not applicable to URLLC. This is because the average delay metric cannot reflect jitter or the packet loss probability caused by delay violations. Even if the average delay is shorter than $1 \mathrm{~ms}$, the delay experienced by a certain packet could be much longer than $1 \mathrm{~ms}$.

2) Hard Deadline: If a service requires a hard deadline $T_{\mathrm{d}}$, it means that the packets should be transmitted to the receiver before the deadline with probability one. To illustrate how to guarantee a hard deadline, we consider a fluid model in a FirstCome-First-Serve (FCFS) system as that in [148]. The total amount of data arriving at the buffer during the time interval $[0, t]$ is defined as $A(t)=\int_{0}^{t} a(\tau) d \tau$. The total amount of data leaving the buffer during time interval $[0, t]$ is denoted by $S(t)=\int_{0}^{t} s(\tau) d \tau$, where $s(t)$ is the service rate at time $t$. To satisfy the hard deadline $T_{\mathrm{d}}$, the following constraints should be satisfied,

$$
\begin{aligned}
& S(t) \leq A(t), \forall t \in[0, T], \\
& S(t) \geq A\left(t-T_{\mathrm{d}}\right), \forall t \in\left[T_{\mathrm{d}}, T+T_{\mathrm{d}}\right],
\end{aligned}
$$

where $T$ is the total service time. Constraint (4) ensures that the amount of data transmitted by the system does not exceed the amount of data that has arrived at the buffer. Constraint (5) ensures the hard deadline requirement.

The hard deadline is widely applied in wireline communications, where the channel is deterministic [148, 158]. However, constraint (5) cannot be satisfied in most wireless communication systems [15].

3) Statistical QoS: For most of the real-time services and URLLC, statistical QoS is the best choice among the three kinds of metrics. Statistical QoS is characterized by a delay bound and a threshold of the maximal tolerable delay bound violation probability, $\left(D_{\mathrm{q}}^{\max }, \varepsilon_{\mathrm{q}}\right)$, respectively. For Voice over Internet Protocol services, the requirement in radio access networks is (50 ms, 2\%) [159]. For URLLC, the queuing delay bound should be less than the E2E delay and the queuing delay violation probability should be less than the overall packet loss probability, e.g., $\left(1 \mathrm{~ms}, 10^{-7}\right)$ [1].

The existing publications on the statistical QoS or the distribution of queuing delay mainly focus on specific arrival models or service models. Some useful results with FCFS servers and Processor-Sharing (PS) servers are summarized in Table III] where Kendall's Notation indicates arrival types, service types, number of servers, and scheduling principles. Here " $M$ " and " $D$ " represent memoryless processes and deterministic processes, respectively. "G" means that the interarrival time between packets or the service time of each packet may follow any general distribution. The abbreviation "Geo ${ }^{[X] " ~ i n ~[160] ~ m e a n s ~ t h a t ~ t h e ~ p a c k e t s ~ a r r i v e d ~ i n ~ a ~ t i m e ~ s l o t ~}$ constitutes a bulk, and the bulk arrival process is stationary and memoryless with Binomial marginal distribution. Considering that PS servers are widely deployed in computing systems [25], A. P. Zwart et al. derived the distribution of the delay in the large-delay regime, which is referred to as a tail probability [161]. Since the delay experienced by short packets in the short delay regime is more relevant to URLLC, C. She et al. derived an approximation of the delay experienced by short packets in a PS server [91].

TABLE III Statistical QoS of SPECIFIC QUeUING Models

\begin{tabular}{|l|l|l|}
\hline Reference & Kendall's Notation & Result \\
\hline$[162]$ & $\mathrm{M} / \mathrm{D} / 1 / \mathrm{FCFS}$ & Distribution of delay \\
\hline$[163]$ & $\mathrm{M} / \mathrm{M} / 1 / \mathrm{FCFS}$ & Distribution of delay \\
\hline$[160]$ & $\mathrm{Geo}^{[X]} / \mathrm{G} / 1 / \mathrm{FCFS}$ & $\begin{array}{l}\text { Upper bound of delay vio- } \\
\text { lation probability }\end{array}$ \\
\hline$[161]$ & $\mathrm{M} / \mathrm{G} / 1 / \mathrm{PS}$ & Tail probability of delay \\
\hline$[91]$ & $\mathrm{M} / \mathrm{G} / 1 / \mathrm{PS}$ & $\begin{array}{l}\text { Delay experienced by short } \\
\text { packets }\end{array}$ \\
\hline
\end{tabular}

There are two principal tools for analyzing statistical QoS in more general queuing systems, i.e., network calculus and effective bandwidth/capacity [151, 152, 164].

The basic idea of network calculus is to convert the accumulated arrival and service rates from the bit domain to the SNR domain, and then derive the upper bounds of the Mellin transforms of the arrival and service processes [165]. From 
the upper bounds of the Mellin transforms, the upper bound of the delay violation probability can be obtained [156].

Network calculus provides the upper bound of the Complementary Cumulative Distribution Function (CCDF) of the steady-state queuing delay. However, network calculus is not convenient in wireless network design since it is very challenging to derive the relation between $\left(D_{\mathrm{q}}^{\max }, \varepsilon_{\mathrm{q}}\right)$ and the required radio resources in a closed form. In URLLC, we are interested in the tail probability of the queuing delay, i.e., the case that $\varepsilon_{\mathrm{q}}$ is very small. Thus, there is no need to derive the upper bound of CCDF for all values of $\varepsilon_{\mathrm{q}}$.

To analyze the asymptotic case when $\varepsilon_{\mathrm{q}}$ is very small, effective bandwidth and effective capacity can be used [151, 152]. Based on Mellin transforms in network calculus, we can derive the effective bandwidth of arrival processes and effective bandwidth of service processes with Gärtner-Ellis theory [151, 152, 165].

Effective bandwidth is defined as the minimal constant service rate that is required to guarantee $\left(D_{\mathrm{q}}^{\max }, \varepsilon_{\mathrm{q}}\right)$ for a random arrival process $a(t)$ [151]. Inspired by the concept of effective bandwidth, D. Wu et al. introduced the concept of effective capacity over wireless channels [152]. Effective capacity is defined as the maximal constant arrival rate that can be served by a random service process $s(t)$ subject to the requirement $\left(D_{\mathrm{q}}^{\max }, \varepsilon_{\mathrm{q}}\right)$. We denote the effective bandwidth of $a(t)$ and the effective capacity of $s(t)$ as $E_{B}$ and $E_{C}$, respectively. When both the arrival process and the service process are random, $\left(D_{\mathrm{q}}^{\max }, \varepsilon_{\mathrm{q}}\right)$ can be satisfied if [166]

$$
E_{C} \geq E_{B}
$$

The formal definitions of effective bandwidth and effective capacity were summarized in [167], i.e.,

$$
\begin{aligned}
& E_{B}=\lim _{t \rightarrow \infty} \frac{1}{\theta t} \ln \mathbb{E}\left[e^{\theta \int_{0}^{t} a(\tau) d \tau}\right], \\
& E_{C}=-\lim _{t \rightarrow \infty} \frac{1}{\theta t} \ln \mathbb{E}\left[e^{-\theta \int_{0}^{t} s(\tau) d \tau}\right],
\end{aligned}
$$

where $\theta$ is a QoS exponent. The value of $\theta$ decreases with $D_{\mathrm{q}}^{\max }$ and $\varepsilon_{\mathrm{q}}$ according to the following expression [168],

$$
\operatorname{Pr}\left(D_{\mathrm{q}}^{\infty} \geq D_{\mathrm{q}}^{\max }\right) \approx \exp \left(-\theta E_{B} D_{\mathrm{q}}^{\max }\right)=\varepsilon_{\mathrm{q}},
$$

where $D_{\mathrm{q}}^{\infty}$ is the steady-state queuing delay. The approximation in (7) is accurate for the tail probability. For URLLC, the accuracy of (7) has been validated in [16], where a Poisson process, an interrupted Poisson process, or a switched Poisson process is served by a constant service rate (since the delay requirement is shorter than the channel coherence time in most cases in URLLC, the channel is quasi-static and the service rate is constant).

Effective bandwidth and effective capacity have been extensively investigated in the existing literature with different queuing models. Some useful results have been summarized in Table IV A more comprehensive survey on effective capacity in wireless networks is presented by Amjad et al. [169].

\section{Communication Theory Tools}

1) Characterizing Correlations of Multiple Links: To improve the reliability of URLLC in wireless communications,
TABLE IV

USEFUL RESULTS FOR ANALYZING STATISTICAL QOS

\begin{tabular}{|c|c|l|}
\hline \multicolumn{3}{|c|}{ Effective Bandwidth } \\
\hline Year & Reference & Sources \\
\hline 1995 & {$[151]$} & General definition \\
\hline 1996 & {$[170]$} & $\begin{array}{l}\text { Periodic sources, Gaussian sources, ON- } \\
\text { OFF sources, and compound Poisson } \\
\text { sources }\end{array}$ \\
\hline 2016 & {$[171]$} & Interrupted Poisson sources \\
\hline 2018 & {$[16]$} & Poisson sources in closed form \\
\hline \multicolumn{3}{|c|}{} \\
\hline Year & Reference & Channels \\
\hline 2003 & {$[152]$} & General definition \\
\hline 2007 & {$[166$} & ON-OFF channel \\
\hline 2007 & {$[172$} & Nakagami-m fading channel \\
\hline 2010 & {$[173$} & Correlated Rayleigh channel \\
\hline 2013 & {$[174]$} & AWGN, finite blocklength regime \\
\hline 2016 & {$[175$} & $\begin{array}{l}\text { Rayleigh channel, finite blocklength } \\
\text { regime }\end{array}$ \\
\hline
\end{tabular}

we can exploit different types of diversities, such as frequency diversity [176], spatial diversity [177], and interface diversity [178]. The basic idea is to send signals through multiple links in parallel. If one or some of these links experience a low channel quality or congestions, the receiver can still recover the packet in time with the signals from the other links.

For example, $N_{\mathrm{P}}$ copies of one packet are transmitted over $N_{\mathrm{P}}$ paths. The packet loss probability of each path is denoted by $P_{\mathrm{L}}(n), n=1, \ldots, N_{\mathrm{P}}$. If the packet losses of different paths are uncorrelated, then the overall packet loss probability can be expressed as $P_{\mathrm{L}}^{\mathrm{tot}}=\prod_{n=1}^{N_{\mathrm{P}}} P_{\mathrm{L}}(n)$.

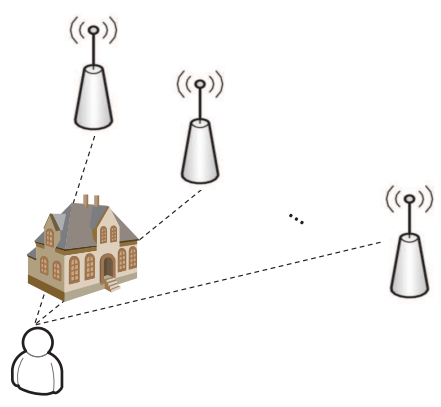

Fig. 6. Cross-correlation of packet losses [24,31].

However, the packet losses of different paths may be highly correlated. To illustrate the impact of cross-correlation of packet losses on the reliability, we consider a multi-antenna system with $N_{\mathrm{P}}$ distributed antennas in Fig. 6 Let $\mathbf{1}_{\mathrm{LoS}}\left(n_{\mathrm{P}}\right)$ be the indicator that the there is a Line-of-Sight (LoS) path between the user and the $n_{\mathrm{P}}$-th antenna. If there is a LoS, $\mathbf{1}_{\mathrm{LoS}}\left(n_{\mathrm{P}}\right)=1$, otherwise, $\mathbf{1}_{\mathrm{LoS}}\left(n_{\mathrm{P}}\right)=0$. Then, the probability that there is a LoS path between the user and each of the distributed antennas can be defined as $P_{\mathrm{LoS}}^{\text {one }} \triangleq \mathbb{E}\left\{\mathbf{1}_{\mathrm{LoS}}\left(n_{\mathrm{P}}\right)\right\}$. When the distances among the $N_{\mathrm{P}}$ antennas are comparable to the typical scale of obstacles (e.g., the widths and lengths of 
buildings) in the environment, the values of $\mathbf{1}_{\mathrm{LoS}}\left(n_{\mathrm{P}}\right), n_{\mathrm{P}}=$ $1, \ldots, N_{\mathrm{P}}$, are correlated. The correlation coefficient of two adjacent antennas is denoted by $\rho_{\mathrm{LoS}}$.

For URLLC, we are interested in the probability that at least one of the antennas can receive the packet from the user. Let's consider a toy example: if there are one or more LoS paths, the antenna can receive the packet, otherwise, the packet is lost. Then, the reliability of the system is the probability that at least one of the antennas has LoS path to the user, i.e. [179],

$$
P_{\mathrm{LoS}}^{\text {all }}=1-\left(1-P_{\mathrm{LoS}}^{\mathrm{one}}\right)\left[1-P_{\mathrm{LoS}}^{\mathrm{one}}\left(1-\rho_{\mathrm{LoS}}\right)\right]^{N_{\mathrm{P}}-1} .
$$

The above expression indicates that $P_{\mathrm{LoS}}^{\text {all }}$ decreases with $\rho_{\mathrm{LoS}}$. If $\rho_{\mathrm{LoS}}=0$, then $P_{\mathrm{LoS}}^{\text {all }}=1-\left(1-P_{\mathrm{LoS}}^{\text {one }}\right)^{N_{\mathrm{P}}}$. If $\rho_{\mathrm{LoS}}=1$, then $P_{\mathrm{LoS}}^{\text {all }}=P_{\mathrm{LoS}}^{\mathrm{one}}$.

For a particular antenna, the value of $P_{\mathrm{LoS}}^{\mathrm{one}}$ depends on the communication environment and the locations of both the antenna and the user. According to $3 \mathrm{GPP}$ LoS model, $P_{\mathrm{LoS}}^{\text {one }}$ in a terrestrial cellular network can be expressed as follows [159],

$$
P_{\mathrm{LoS}}^{\mathrm{one}}=\min \left(\frac{18}{r_{\mathrm{u}}}, 1\right)\left[1-\exp \left(-\frac{r_{\mathrm{u}}}{36}\right)\right]+\exp -\frac{r_{\mathrm{u}}}{36},
$$

where $r_{\mathrm{u}}$ is the distance between the user and the antenna. For ground-to-air channels, the value of $P_{\mathrm{LoS}}^{\text {one }}$ depends on the elevation angle according to the following expression [180, 181],

$$
P_{\mathrm{LoS}}^{\mathrm{one}}=\frac{1}{1+\phi_{\mathrm{e}} \exp \left[-\psi_{\mathrm{e}}\left(\theta_{\mathrm{u}}-\phi_{\mathrm{e}}\right)\right]},
$$

where $\theta_{\mathrm{u}}$ is the elevation angle of the user, and $\phi_{\mathrm{e}}$ and $\psi_{\mathrm{e}}$ are two constants, which depend on the communication environment, such as suburban, urban, dense urban, and highrise urban. The typical values of $\phi_{\mathrm{e}}$ and $\psi_{\mathrm{e}}$ can be found in [31].

In practice, the packet loss probability depends not only on whether there is a LoS path but also on the shadowing of a wireless channel. To characterize shadowing correlation, S. Szyszkowicz et al. established useful shadowing correlation models in [182]. Even with these models, deriving the packet loss probability in URLLC is very challenging. To overcome this difficulty, a numerical method for evaluating packet loss probability was proposed by D. Öhmann et al. [183]. More recently, C. She et al. analyzed the impact of shadowing correlation on the network availability, where a packet is transmitted via a cellular link and a Device-to-Device (D2D) link [184].

Furthermore, the correlation of multiple subchannels in MIMO channels and frequency-selective channels also affects micro-diversity gains. The achievable rate of massive MIMO systems with spatial correlation was investigated in [185], where the authors maximized the data rate over correlated channels. The reliability over frequency-selective Rayleigh fading channels was considered in [176], where an approximation of the outage probability over correlated channels was derived. Considering that there are very few results on the reliability over correlated channels, how to guarantee QoS requirements of URLLC over correlated channels deserves further research.
2) Stochastic Geometry for Delay Analysis in Large-Scale Networks: Most of the existing publications on delay analysis consider the systems with small scale and deterministic network topologies. However, in practical systems, the locations of a large number of users are stochastic. Users can either communicate with each other via D2D links or communicate with BSs via cellular links. Caused by dynamic traffic loads, network congestions lead to a significant delay in large-scale wireless networks with high user density. Yet, how to analyze delay, especially in the low-latency regime, remains an open problem.

In 2012, M. Haenggi applied stochastic geometry to analyze delays in large-scale networks [186], where the average access delay was derived. One year later, P. Kong studied the tradeoff between the power consumption and the average delay experienced by packets [187], where existing results on $\mathrm{M} / \mathrm{G} / 1$ queue were fitted within the stochastic geometry framework. More recently, Y. Zhong et al. proposed a notation of delay outage to evaluate the delay performance of different scheduling policies, defined as the probability that the average delay experienced by a typical user is longer than a threshold conditioned on the spatial locations of BSs, i.e. [188],

$$
\operatorname{Pr}\left\{\bar{D}>T_{\mathrm{th}} \mid \Phi_{\mathrm{b}}\right\},
$$

where $\bar{D}$ is the mean delay experienced by the user, $T_{\text {th }}$ is the required threshold, and $\Phi_{\mathrm{b}}$ is a realization of spatial locations of BSs.

As discussed in Section IV-B, the requirement of URLLC should be characterized by a constraint on the statistical QoS. However, most of the existing studies analyzed the average delay in large-scale networks. To the best knowledge of the authors, there is no available tool for analyzing statistical QoS in large-scale networks.

\section{Summary of Analytical Tools}

The analytical tools enable us to evaluate the performance of wireless networks without extensive simulations, and serve as the key building blocks of optimization frameworks. Yet, there are some open issues:

- Each of these tools is used to analyze the performance of one part of a wireless network. A whole picture of the system is needed for E2E optimization, which is analytically intractable in most of the cases.

- To apply these tools, we need simplified models and ideal assumptions. The impacts of model mismatch on the performance of URLLC remain unclear. For example, if Rayleigh fading (without LoS path between transmitters and receivers) is adopted in analytical, one can obtain an upper bound of the latency or the packet loss probability (The LoS component exists in practical communication scenarios). However, such a model will lead to conservative resource allocation.

- The tools in Tables [II III and IV rely on simplified channel and queuing models. For more complicated and practical models, we can hardly derive the tail probability of delay and the packet loss probability. 


\begin{tabular}{|c|c|c|}
\hline Physical Layer Solutions & Link Layer Solutions & Network Layer Solutions \\
\hline Short Frame Structure & Retransmission and Repetition & MEC \\
\hline Short Channel Codes & DL Schedulers & Anticipatory Networking \\
\hline Massive MIMO, mmWave & UL Grant-free Access & SDN and Network Slicing \\
\hline$\ldots$ & $\ldots$ & $\ldots$ \\
\hline
\end{tabular}

Selecting solutions from each layer to obtain cross-layer solutions

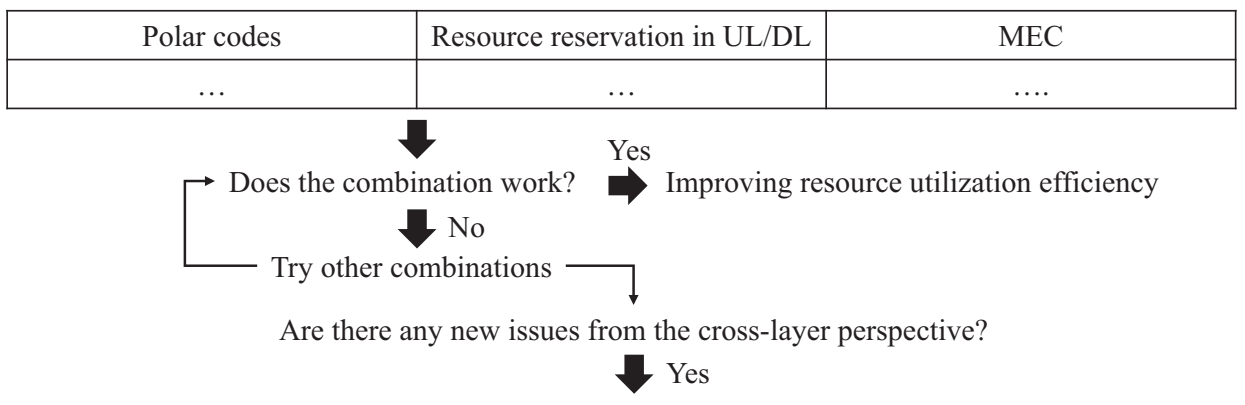

How to address these issues? What's the performance limit? How to approach it?

Fig. 7. From single-layer design to cross-layer design.

To address the above issues, we will illustrate how to optimize the whole network with cross-layer design in Section D, introduce deep transfer learning to handle model mismatch in Section VI] and discuss model-free approaches in Sections VII and VIII

\section{Cross-Layer Design}

By decomposing communication systems into seven layers [14], we can develop practical, but suboptimal solutions for URLLC in each layer. To reflect the interactions among different layers, and to optimize the delay and reliability of the whole system, we turn to cross-layer optimization. Although cross-layer design in wireless networks has been studied in the existing literature, the solutions for URLLC are limited. The most recent works on cross-layer design for URLLC are summarized in Table $\mathrm{V}$

\section{A. Design Principles and Fundamental Results}

1) Design Principles: The design principles in the crosslayer design are shown in Fig. 7 A straightforward approach is selecting technologies from each layer, and see whether this combination works. If the combination can guarantee the QoS requirement of URLLC, then we can further optimize resource allocation to improve the resource utilization efficiency. Otherwise, we need to try some other combinations. Meanwhile, we may identify new issues from a cross-layer perspective. By cross-layer optimization, we aim to find out the performance limit and show how to approach the performance limit.

2) Fundamental Results: R. Berry et al. investigated how to guarantee average delay constraints over fading channels [143]. They obtained the Pareto optimal power-delay tradeoff, i.e., the average transmit power decreases with the average queuing delay according to $1 / \bar{D}^{2}$ in the large delay regime. To achieve the optimal tradeoff, a power control policy should take both Channel State Information (CSI) and Queue State
Information (QSI) into account. If we apply any power control policy that does not depend on QSI, such as the waterfilling policy [24], then the average transmit power decreases with the delay according to $1 / \bar{D}$. In other words, directly combining the water-filling policy (i.e., the optimal policy that maximizes the average throughput in the physical layer) with a queuing system cannot achieve the optimal tradeoff between the average transmit power and the average queuing delay.

Following this fundamental result, M. J. Neely extended the power-delay tradeoff into multi-user scenarios [144] and proposed a packet dropping mechanism that can exceed the Pareto optimal power-delay tradeoff [199]. Considering that the average delay metric is not suitable for real-time services, such as video and audio, an optimal power control policy that maximizes the throughput subject to the statistical QoS requirement was derived by J. Tang et al. in [172]. The result in [172] shows that when the required delay bound goes to infinite, the optimal power control policy converges to the water-filling policy in [24]. When the required delay bound approaches the channel coherence time, the optimal power control policy converges to the channel inverse.

\section{B. Physical- and Link-Layer Optimization}

A diagram of cross-layer optimization for physical and link layers is shown in Fig. 8 Since the E2E delay requirement of URLLC is around $1 \mathrm{~ms}$, the required delay bound is shorter than the channel coherence time [200]. To guarantee a queuing delay that is shorter than the channel coherence time, the power control policy should be the channel inverse [172]. For typical wireless channels, such as Rayleigh fading or Nakagami- $m$ fading, the required transmit power of the channel inverse is unbounded.

The issue observed from cross-layer design is similar to the outage probability defined in [58]. Different from the physicallayer analysis, outage probability does not equal to the packet 
TABLE V

CROSS-LAYER OPTIMIZATION FOR URLLC

\begin{tabular}{|c|c|c|c|c|}
\hline Design Objective & Physical-layer issues & Link-layer issues & Network-layer issues & Challenges/Results \\
\hline $\begin{array}{l}\text { Power control opti- } \\
\text { mization [174] }\end{array}$ & $\begin{array}{l}\text { Normal approximation over } \\
\text { AWGN channel }\end{array}$ & $\begin{array}{l}\text { Ensuring the statistical QoS with } \\
\text { effective capacity }\end{array}$ & N/A & $\begin{array}{l}\text { No closed-form expression } \\
\text { in the short blocklength } \\
\text { regime }\end{array}$ \\
\hline $\begin{array}{l}\text { Throughput analysis } \\
\text { [189 }\end{array}$ & $\begin{array}{l}\text { Normal approximation in cog- } \\
\text { nitive radio systems }\end{array}$ & $\begin{array}{l}\text { Ensuring the statistical QoS with } \\
\text { effective capacity }\end{array}$ & N/A & $\begin{array}{l}\text { No closed-form result in } \\
\text { general }\end{array}$ \\
\hline $\begin{array}{ll}\text { Delay analysis } \\
\text { [156 }\end{array}$ & $\begin{array}{l}\text { Normal approximation over } \\
\text { quasi-static fading channel }\end{array}$ & $\begin{array}{l}\text { Analyzing the statistical QoS with } \\
\text { network calculus }\end{array}$ & N/A & $\begin{array}{l}\text { No closed-form result in } \\
\text { general }\end{array}$ \\
\hline $\begin{array}{l}\text { Packet scheduling } \\
\text { [15] }\end{array}$ & $\begin{array}{l}\text { Normal approximation over } \\
\text { quasi-static fading channel }\end{array}$ & $\begin{array}{l}\text { DL packet scheduling before a hard } \\
\text { deadline }\end{array}$ & N/A & $\begin{array}{l}\text { An online algorithm with } \\
\text { small performance losses }\end{array}$ \\
\hline $\begin{array}{l}\text { Throughput analysis } \\
\text { [175] }\end{array}$ & $\begin{array}{l}\text { Throughput of relay systems } \\
\text { the in short blocklength regime }\end{array}$ & $\begin{array}{l}\text { Ensuring the statistical QoS with } \\
\text { effective capacity }\end{array}$ & N/A & $\begin{array}{l}\text { No closed-form result in } \\
\text { general }\end{array}$ \\
\hline $\begin{array}{l}\text { Power minimization } \\
\text { [16 }\end{array}$ & $\begin{array}{l}\text { Normal approximation over } \\
\text { quasi-static fading channel }\end{array}$ & $\begin{array}{l}\text { Ensuring the statistical QoS with } \\
\text { effective bandwidth }\end{array}$ & N/A & $\begin{array}{l}\text { Near-optimal solutions of the } \\
\text { non-convex problem }\end{array}$ \\
\hline $\begin{array}{l}\text { Improving the } \\
\text { reliability-SE trade- } \\
\text { off [178] }\end{array}$ & N/A & $\begin{array}{l}\text { Improving the tradeoff between } \\
\text { spectrum efficiency and reliability } \\
\text { with network coding }\end{array}$ & $\begin{array}{l}\text { Packet cloning and split- } \\
\text { ting on multiple commu- } \\
\text { nication interfaces }\end{array}$ & $\begin{array}{l}\text { Intractable in large-scale net- } \\
\text { work }\end{array}$ \\
\hline $\begin{array}{l}\text { Improving reliabil- } \\
\text { ity [190] }\end{array}$ & $\begin{array}{l}\text { Decoding errors in short block- } \\
\text { length regime }\end{array}$ & $\begin{array}{l}\begin{array}{l}\text { Scheduler design under a hard } \\
\text { deadline constraint }\end{array} \\
\end{array}$ & N/A & $\begin{array}{l}\text { Reducing outage probability } \\
\text { with combination strategies }\end{array}$ \\
\hline $\begin{array}{l}\text { Bandwidth } \\
\text { minimization } \\
{[191]}\end{array}$ & $\begin{array}{l}\text { Achievable rate in short block- } \\
\text { length regime }\end{array}$ & $\begin{array}{l}\text { E2E optimization (i.e., UL/DL } \\
\text { transmission delay and queuing de- } \\
\text { lay) }\end{array}$ & N/A & $\begin{array}{l}\text { Near-optimal solutions of the } \\
\text { non-convex problem }\end{array}$ \\
\hline $\begin{array}{l}\text { Power allocation } \\
{[17]}\end{array}$ & $\begin{array}{l}\text { Maximizing the sum through- } \\
\text { put of multiple users in short } \\
\text { blocklength regime }\end{array}$ & $\begin{array}{l}\text { Ensuring statistical QoS for differ- } \\
\text { ent kinds of packet arrivals }\end{array}$ & N/A & $\begin{array}{l}\text { A sub-optimal algorithm for } \\
\text { solving the non-convex prob- } \\
\text { lem }\end{array}$ \\
\hline $\begin{array}{l}\text { Bandwidth } \\
\text { minimization } \\
\text { 192 }\end{array}$ & $\begin{array}{l}\text { Normal approximation over } \\
\text { quasi-static fading channel }\end{array}$ & $\begin{array}{l}\text { Burstiness aware resource reser- } \\
\text { vation under statistical QoS con- } \\
\text { straints }\end{array}$ & N/A & $\begin{array}{l}\text { Reducing } 40 \% \text { bandwidth by } \\
\text { traffic state prediction }\end{array}$ \\
\hline $\begin{array}{l}\text { Availability analysis } \\
\text { [184 }\end{array}$ & $\begin{array}{l}\text { Normal approximation over } \\
\text { quasi-static fading channel }\end{array}$ & $\begin{array}{l}\text { Different transmission protocols } \\
\text { via cellular links and D2D links }\end{array}$ & 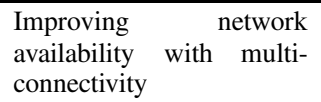 & $\begin{array}{l}\text { An accurate approximation } \\
\text { of network availability }\end{array}$ \\
\hline $\begin{array}{l}\text { Coding-queuing } \\
\text { analyses [160] }\end{array}$ & $\begin{array}{l}\text { Variable-length-stop-feedback } \\
\text { codes }\end{array}$ & $\begin{array}{l}\text { Retransmission under latency and } \\
\text { peak-age violation guarantees }\end{array}$ & N/A & $\begin{array}{l}\text { Accurate approximations of } \\
\text { target tail probabilities }\end{array}$ \\
\hline $\begin{array}{l}\text { Coded } \\
\text { retransmission } \\
\text { design [193] }\end{array}$ & Tiny codes with just 2 packets & $\begin{array}{l}\text { Automatic Repeat Request (ARQ) } \\
\text { protocols with delayed and unreli- } \\
\text { able feedback }\end{array}$ & N/A & $\begin{array}{l}\text { Improving } 40 \% \text { throughput } \\
\text { with latency and reliability } \\
\text { guarantees }\end{array}$ \\
\hline $\begin{array}{l}\text { Optimizing resource } \\
\text { allocation [18] }\end{array}$ & $\begin{array}{l}\text { Ensuring transmission delay in } \\
\text { the short blocklength regime }\end{array}$ & N/A & $\begin{array}{l}\text { Network slicing } \\
\text { eMBB and } \\
\text { cloud-RAN }\end{array}$ & $\begin{array}{l}\text { Searching near-optimal solu- } \\
\text { tions of mixed-integer pro- } \\
\text { gramming }\end{array}$ \\
\hline $\begin{array}{ll}\text { Delay analysis } \\
{[194]}\end{array}$ & $\begin{array}{l}\text { Multi-user MISO with imper- } \\
\text { fect CSI and finite blocklength } \\
\text { coding }\end{array}$ & $\begin{array}{l}\text { Analyzing statistical QoS with net- } \\
\text { work calculus }\end{array}$ & N/A & $\begin{array}{l}\text { No closed-form solution in } \\
\text { general }\end{array}$ \\
\hline $\begin{array}{l}\text { Delay, reliability, } \\
\text { throughput analysis } \\
\text { [195] }\end{array}$ & Modulation and coding scheme & $\begin{array}{l}\text { Incremental redundancy-hybrid } \\
\text { ARQ over correlated Rayleigh } \\
\text { fading channel }\end{array}$ & N/A & $\begin{array}{l}\text { No closed-form solution in } \\
\text { general }\end{array}$ \\
\hline $\begin{array}{l}\text { Resource allocation } \\
\text { [196] }\end{array}$ & Modulation and coding scheme & $\begin{array}{l}\text { Individual resource reservation or } \\
\text { contention-based resource sharing }\end{array}$ & N/A & $\begin{array}{l}\text { Analytical expressions of re- } \\
\text { liability }\end{array}$ \\
\hline $\begin{array}{l}\text { Spectrum } \begin{array}{r}\text { and } \\
\text { power allocation } \\
{[19]}\end{array} \\
19\end{array}$ & $\begin{array}{l}\text { Interference management for } \\
\text { vehicle-to-vehicle and vehicle- } \\
\text { to-infrastructure links }\end{array}$ & $\begin{array}{l}\text { Ensuring statistical QoS with effec- } \\
\text { tive capacity }\end{array}$ & N/A & $\begin{array}{l}\text { Solving subproblems on } \\
\text { spectrum/power allocation } \\
\text { in polynomial time }\end{array}$ \\
\hline $\begin{array}{l}\text { Distortion } \\
\text { minimization } \\
\text { 197 }\end{array}$ & Joint source and channel codes & N/A & $\begin{array}{l}\text { Improving reliability over } \\
\text { parallel AWGN channels }\end{array}$ & $\begin{array}{l}\text { Tight approximations and } \\
\text { bounds on distortion level, } \\
\text { but not in closed form }\end{array}$ \\
\hline $\begin{array}{l}\text { Network } \\
\text { architecture design } \\
{[198]}\end{array}$ & CoMP communications & $\begin{array}{l}\text { E2E design of } 5 \mathrm{G} \text { networks for } \\
\text { industrial factory automation }\end{array}$ & $\begin{array}{l}\text { A novel architecture for } \\
\text { TSN }\end{array}$ & $\begin{array}{l}\text { Around } 2 \mathrm{~ms} \text { round-trip de- } \\
\text { lay in their prototype }\end{array}$ \\
\hline $\begin{array}{l}\text { Offloading } \\
\text { optimization }\end{array}$ & $\begin{array}{l}\text { Normal approximation over } \\
\text { quasi-static fading channel }\end{array}$ & $\begin{array}{l}\text { Delay analysis in processor-sharing } \\
\text { servers with both short and long } \\
\text { packets }\end{array}$ & $\begin{array}{l}\text { User association \& task } \\
\text { offloading of mission- } \\
\text { critical IoT in MEC }\end{array}$ & $\begin{array}{l}\text { Closed-form approximations } \\
\text { of delay and low-complexity } \\
\text { offloading algorithms }\end{array}$ \\
\hline
\end{tabular}



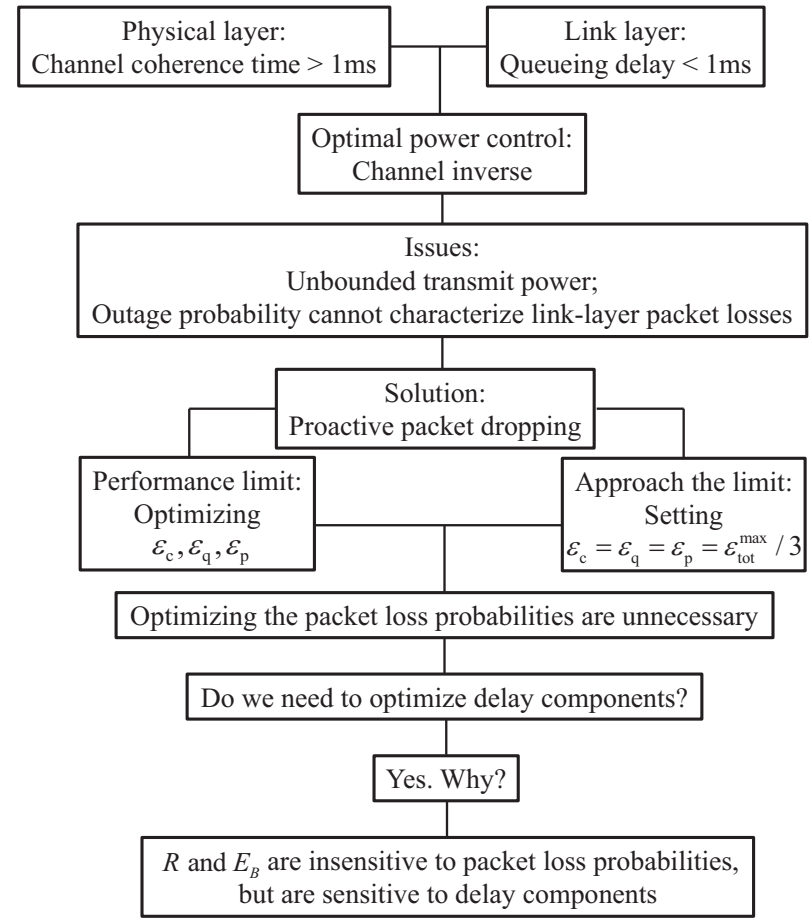

Fig. 8. Diagram of physical- and link-layer optimization [16 69.

loss probability in cross-layer design. As shown in [16], by dropping some packets proactively, it is possible to reduce the overall packet loss probability. Such an idea was inspired by the result in [199]. Denote the proactive packet dropping probability by $\varepsilon_{\mathrm{p}}$. By optimizing decoding error probability, queuing delay violation probability, and proactive packet dropping probability, we can minimize the required maximal transmit power at the cost of high computational complexity. To avoid complicated optimization, a straightforward solution is setting $\varepsilon_{\mathrm{c}}=\varepsilon_{\mathrm{q}}=\varepsilon_{\mathrm{p}}=\varepsilon_{\mathrm{tot}}^{\max } / 3$. The results in [16] show that the performance loss (i.e., required transmit power) without optimization is less than $5 \%$ when the number of antennas is larger than eight. In other words, setting the packet loss probabilities as equal is near-optimal, and there is no need to optimize the values of $\varepsilon_{\mathrm{c}}, \varepsilon_{\mathrm{q}}$, and $\varepsilon_{\mathrm{p}}$.

From the above-mentioned conclusion, it is natural to raise the following question: Is it necessary to optimize the delay components subject to the E2E delay? The results in [191] show that by optimizing UL/DL delays and queuing delay subject to the E2E delay constraint, a large amount of bandwidth can be saved. This is because the achievable rate in the short blocklength regime is insensitive to the decoding error probability, but very sensitive to transmission delay. Therefore, optimizing the delay components subject to the E2E delay requirement is necessary for maximizing resource utilization efficiencies of URLLC.

Apart from the above solutions, some other cross-layer solutions for physical and link layers have been developed in URLLC recently. By considering the hard deadline metric, an energy-efficient scheduling policy for short packet transmission was proposed in [15], and delay bound violation probabilities of different schedulers were evaluated in [190].
How to guarantee statistical QoS in the short blocklength regime has been studied in UL Tactile Internet [192], DL multi-user scenarios with Markovian sources [17], point-topoint communications with imperfect CSI [194], and vehicle networks with strong interferences between vehicle-to-vehicle and vehicle-to-infrastructure links [19]. Coded ARQ and incremental redundancy-hybrid ARQ schemes were developed in [193] and [195], respectively. Considering that the density of devices in smart factory can be very large, the individual resource reservation scheme and the contention-based resource sharing scheme were considered in [196].

\section{Physical-, Link-, and Network-Layer Optimization}

The cross-layer models will be very complicated if more than two layers are considered. Thus, joint optimization of physical, link, and network layers is very challenging. Although a few papers optimize network management from a cross-layer approach, some simplified models on the physical and link layers are used in these works [18, 178, 198].

When optimizing packet splitting on multiple communication interfaces, the authors of [178] assumed that the distribution of latency (i.e., the latency-reliability function) of each interface is available. With the simplified link-layer model, coded packets are distributed through multiple interfaces. As in [178], joint source and channel codes over parallel channels were developed in [198], where simplified AWGN channels with independent fading are considered in the analysis. To optimize network slicing for eMBB and URLLC in cloudRAN, the resources that are required to satisfy the data rate constraint of $\mathrm{eMBB}$ and the transmission delay constraint of URLLC were derived in [18]. The latency in the linklayer was not considered in the framework in [18]. Otherwise, the problem will become intractable. Resource allocation in vehicle networks was optimized to maximize the throughput of URLLC in [201], where Shannon's capacity is used to simplify the complicated achievable rate over interference channel and the average packet loss probability and average latency are analyzed in the queuing system for analytical tractability.

To validate the E2E design in a time-sensitive network architecture with CoMP for URLLC, issues in the three lower layers were considered in [198], where most of the conclusions are obtained via simulation. Since the models are too complicated, one can hardly obtain any closed-form result. To gain some useful insights from theoretical analysis, the authors of [91] analyzed the E2E delay in an MEC system, where the UL and DL transmission delays and the processing delay at the servers are considered. In addition, the packet loss probabilities caused by decoding errors and queuing delay violations were also derived. Based on these physical- and link-layer models, user association and computing offloading of URLLC service were optimized in a multi-cell MEC system, where eMBB services are considered as background services (i.e., not optimized).

\section{From Lower Layers to Higher Layers}

The E2E performance of URLLC depends on all the seven layers in the OSI model [202]. By considering more layers in communication system design, better performance 
can be achieved. However, most of the working groups in standardization mainly focus on one or two layers [74]. For example, the Institute of Electronics and Electrical Engineers (IEEE) and the Internet Engineering Task Force (IETF) have formed some working groups on the standardization of TSN [203]. The IEEE 802.1 working group mainly focuses on the physical and link layers, while the deterministic network working group of IETF focuses on the network layer and higher layers [74]. By including more protocol layers in one design framework, the problem will become more complicated, but the obtained policy has the potential to achieve a better performance. Essentially, there is a tradeoff between complexity and performance. To move from the three lower layers to higher layers, novel technologies that can capture complicated features and can be implemented in real-time are in urgent need. For example, driven by the requirement from the application layer, the freshness of the knowledge becomes one of the KPIs in remote monitoring/control systems [48]. To achieve a better tradeoff between latency and AoI, the authors of [160] developed variable-length-stop-feedback codes that outperform the simple automatic repetition request in terms of the delay violation probability. In addition, their results indicated that by changing the service order of a queuing system, it is possible a smaller peak-age violation probability.

\section{E. Summary of Cross-layer Optimization}

From a cross-layer optimization framework, one can discover new research challenges in URLLC. By solving crosslayer optimization problems, the performance limits on latency, reliability, and resource utilization efficiency can be obtained. In addition, the optimal policies provide some useful insights on how to find near-optimal solutions and how to approach the performance limits. Some open issues of crosslayer optimization are summarized as follows:

- The problem formulation relies on the analytical tools in Section IV Thus, some open issues of the analytical tools, such as model mismatch, also exist in the optimization frameworks.

- The optimization problems are NP-hard in most cases. The optimal policies or fast optimization algorithms are developed on a case by case basis. A general methodology for designing fast algorithms is missing.

- The results obtained in the existing literature (See Table V) only considered two or three bottom layers. Thus, these frameworks cannot address the technical problems in the upper layers. If the issues in the upper layers are taken into account, the optimization problems could be intractable.

We will discuss how to take advantage of existing domain knowledge and how to transfer the knowledge to practical scenarios in the rest part of this paper.

\section{SUPERVISED DEEP LEARNING FOR URLLC}

Deep learning is a family of machine learning techniques based on DNNs. Depending on the training methods, it can be categorized as supervised, unsupervised, and reinforcement learning. Supervised deep learning has been applied in different fields in computer science, including computer vision [204], speech recognition [205], natural language processing [206], and so on. Recently, supervised deep learning algorithms were also adopted in wireless networks [207]. The existing publications that used supervised deep learning for channel esimation/prediction, traffic prediction, mobility/trajectory prediction, QoS/Quality-of-Experience (QoE) prediction, and policy approximation are summarized in Table VI. Although the algorithms in this table are not designed for URLLC, they can be applied in the prediction and communication co-design framework for URLLC in [192].

\section{A. Basics of DNNs}

In practice, a complicated DNN is usually made up of some basic structures, such as feed-forward neural networks (FNNs), recurrent neural networks (RNNs), and convolutional neural networks (CNNs) in Fig. [226]. How to build a DNN for a specific problem depends on the structure of data. In this section, we take FNNs as an example to introduce some basics of DNNs. The motivation is to provide a quick review of DNN. In general, FNNs work well with small-scale problems. For an in-depth introduction of different kinds of DNNs, please refer to [20].

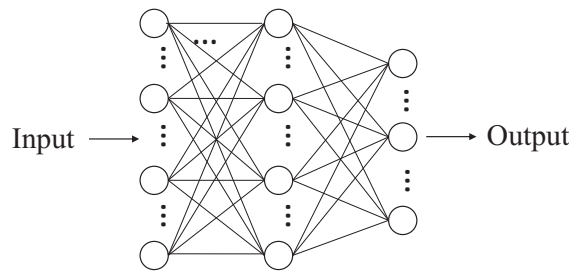

(a) FNN

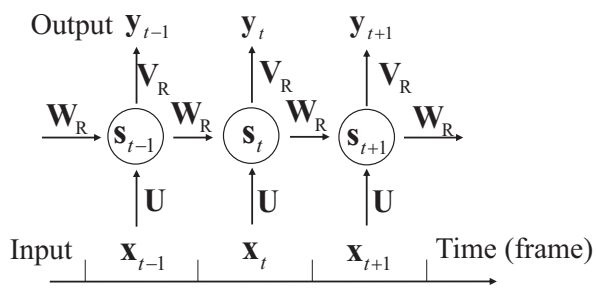

(b) RNN

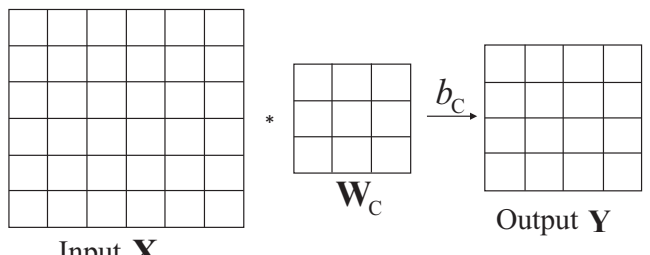

(c) A single-layer CNN

Fig. 9. Different kinds of neural networks [226].

1) Basics of FNN: An FNN includes an input layer, an output layer, and $N_{L}$ hidden layers [226]. The output of the $n$-th layer is a column vector $\mathbf{x}^{[n]} \in \mathbb{R}^{M^{[n]} \times 1}$, where $n=$ $0,1, \ldots, N_{L}+1$ and $M^{[n]}$ is the number of neurons in the $n$-th layer. For the input layer, $n=0$. For the output layer, $n=$ 
TABLE VI

SUPERVISED DEEP LEARNING IN WIRELESS NETWORKS

\begin{tabular}{|c|c|c|c|c|}
\hline \multicolumn{5}{|c|}{ Channel Estimation or Prediction } \\
\hline Reference & Summary & Accuracy & Complexity & System Performance \\
\hline 208 & $\begin{array}{l}\text { Proposing a multiple-rate compressive sensing deep learning } \\
\text { framework for CSI feedback in massive MIMO systems }\end{array}$ & $\begin{array}{l}\text { Relative error: below } \\
0.01\end{array}$ & $\begin{array}{l}\text { Linear in the size of } \\
\text { the channel matrix }\end{array}$ & Not available \\
\hline 209 & $\begin{array}{l}\text { Employing CNN for wideband channel estimation in } \\
\text { mmWave massive MIMO systems }\end{array}$ & $\begin{array}{l}\text { Relative error: below } \\
0.01\end{array}$ & $\begin{array}{l}\text { Lower than Minimum } \\
\text { Mean Square Error } \\
\text { (MMSE) }\end{array}$ & Not available \\
\hline [210] & $\begin{array}{l}\text { Applying Long Short Term Memory (LSTM) to make } \\
\text { channel predictions in body area networks }\end{array}$ & $\begin{array}{l}\text { Relative error: below } \\
0.05\end{array}$ & Not available & $\begin{array}{l}\text { Outage probability: } \\
1 \sim 10 \%\end{array}$ \\
\hline 211 & $\begin{array}{l}\text { Predicting future signal strength with LSTM and gated } \\
\text { recurrent unit algorithms }\end{array}$ & $\begin{array}{l}\text { Better than linear regres- } \\
\text { sion }\end{array}$ & Not available & Not available \\
\hline \multicolumn{5}{|c|}{$\begin{array}{ll}\text { Traffic Prediction } \\
\end{array}$} \\
\hline Reference & Summary & Accuracy & Complexity & System Performance \\
\hline 212 & $\begin{array}{l}\text { Proposing a hybrid deep learning model for spatiotemporal } \\
\text { traffic prediction in cellular networks }\end{array}$ & $\begin{array}{l}\text { Reduce } 20 \sim 40 \% \text { pre- } \\
\text { diction error }\end{array}$ & Not available & Not available \\
\hline 213 & $\begin{array}{l}\text { Applying RNN in spatiotemporal traffic prediction in cellu- } \\
\text { lar networks }\end{array}$ & $\begin{array}{l}\text { The n-to-n RNN outper- } \\
\text { forms other schemes }\end{array}$ & Not available & Not available \\
\hline$[214$ & $\begin{array}{l}\text { Comparing the performance of statistic learning and deep } \\
\text { learning methods in user traffic prediction }\end{array}$ & $\begin{array}{l}\text { LSTM is better than } \\
\text { other schemes }\end{array}$ & Not available & $\begin{array}{l}45 \% \text { decrease in de- } \\
\text { lay }\end{array}$ \\
\hline [215 & $\begin{array}{l}\text { Developing deep learning architecture for traffic prediction } \\
\text { and applying transfer learning among different scenarios }\end{array}$ & $\begin{array}{l}5 \sim 20 \% \text { improvements } \\
\text { in different datasets }\end{array}$ & Not available & Not available \\
\hline \multicolumn{5}{|c|}{$\begin{array}{ll}\text { Mobility or Trajectory Prediction } \\
\end{array}$} \\
\hline Reference & Summary & Accuracy & Complexity & System Performance \\
\hline 216 & $\begin{array}{l}\text { Comparing LSTM, echo state network, and an optimal } \\
\text { linear predictor, and Kalman filter, in trajectory prediction }\end{array}$ & $\begin{array}{l}\text { LSTM is better than } \\
\text { other schemes }\end{array}$ & Not available & Not available \\
\hline 217 & $\begin{array}{l}\text { Comparing FNN, Extreme Gradient Boosting Trees (XG- } \\
\text { Boost), and two other benchmarks in location prediction }\end{array}$ & $\begin{array}{l}\text { XGBoost is better than } \\
\text { other schemes (error } \\
\text { probability: }<10 \% \text { ) }\end{array}$ & $\begin{array}{l}\text { Semi-Markov-based } \\
\text { model has the lowest } \\
\text { complexity }\end{array}$ & $\begin{array}{l}\text { XGBoost performs } \\
\text { best }(80.68 \% \text { energy } \\
\text { saving) } \\
\end{array}$ \\
\hline$[218$ & $\begin{array}{l}\text { Applying RNN in unmanned aerial vehicle trajectory pre- } \\
\text { diction }\end{array}$ & $\begin{array}{l}\text { Almost } 98 \% \text { prediction } \\
\text { accuracy }\end{array}$ & Not available & Not available \\
\hline [219] & $\begin{array}{l}\text { Applying LSTM in head and body motion prediction to } \\
\text { reduce latency in VR applications }\end{array}$ & $\begin{array}{l}\text { Prediction error: }<1^{\circ} \\
\text { and }<1 \mathrm{~mm}\end{array}$ & Not available & $\begin{array}{l}\text { Mismatched pixels: } \\
1 \sim 3 \%\end{array}$ \\
\hline \multicolumn{5}{|c|}{ QoS or QoE Prediction } \\
\hline Reference & Summary & Accuracy & Complexity & System Performance \\
\hline 220 & $\begin{array}{l}\text { Proposing a combination of } \mathrm{CNN}, \mathrm{RNN} \text {, and Gaussian } \\
\text { process classifier for a binary QoE prediction }\end{array}$ & $\begin{array}{l}\text { From } 60 \% \text { to } 90 \% \text { de- } \\
\text { pending on datasets }\end{array}$ & Not available & Not available \\
\hline 221 & Applying LSTM in QoS prediction for IoT applications & $\begin{array}{l}\text { LSTM outperforms } \\
\text { other schemes }\end{array}$ & Not available & Not available \\
\hline 222 & $\begin{array}{l}\text { Predicting the processing time of radio and baseband net- } \\
\text { work functions with mathematical and deep learning models }\end{array}$ & $\begin{array}{l}\text { DNN outperforms other } \\
\text { schemes }\end{array}$ & Not available & Not available \\
\hline \multicolumn{5}{|c|}{ Approximating Optimal Policies } \\
\hline Reference & Summary & Accuracy & Complexity & System Performance \\
\hline 223 & $\begin{array}{l}\text { Approximating Weighted Minimum Mean Square Error } \\
\text { (WMMSE) power control scheme with FNN }\end{array}$ & Up to $98 \%$ & $\begin{array}{l}\text { CPU time: } 10 \% \text { of } \\
\text { WMMSE }\end{array}$ & $\begin{array}{l}\text { Near-optimal in } \\
\text { small-scale networks }\end{array}$ \\
\hline [224] & $\begin{array}{l}\text { Using SE/EE as the loss function to train CNNs for power } \\
\text { control }\end{array}$ & Not available & $\begin{array}{l}\text { Lower than FNN and } \\
\text { WMMSE }\end{array}$ & $\begin{array}{l}\text { Higher SE/EE than } \\
\text { WMMSE }\end{array}$ \\
\hline 225 & $\begin{array}{l}\text { Employing an FNN to approximate the optimal predictive } \\
\text { resource allocation policy for delay-tolerant services }\end{array}$ & $\begin{array}{l}70 \sim 90 \% \text { depending } \\
\text { on the density of users }\end{array}$ & $\begin{array}{l}\text { Lower than Interior- } \\
\text { Point algorithm }\end{array}$ & $\begin{array}{l}97 \% \text { of users with } \\
\text { satisfactory QoS }\end{array}$ \\
\hline
\end{tabular}

$N_{L}+1$. The relation between $\mathbf{x}^{[n]}$ and $\mathbf{x}^{[n-1]}$ is determined function, by the following expression,

$$
\mathbf{x}^{[n]}=f_{\mathrm{A}}^{[n]}\left(\mathbf{W}^{[n]} \mathbf{X}^{[n-1]}+\mathbf{b}^{[n]}\right),
$$

where $\mathbf{W}^{[n]} \in \mathbb{R}^{M^{[n]} \times M^{[n-1]}}$ and $\mathbf{b}^{[n]} \in \mathbb{R}^{M^{[n]} \times 1}$ are the weights and bias of the $n$-th layer, and $f_{\mathrm{A}}^{[n]}(\cdot)$ is the activation function of the $n$-th layer, and it is an element-wise operation. The widely used activation functions include the sigmoid

$$
f_{\mathrm{A}}(\mathbf{x})=\frac{1}{1+\exp (\mathbf{x})},
$$

$\tanh (\mathbf{x})$, the Rectified Linear Unit (ReLU) function,

$$
f_{\mathrm{A}}(\mathbf{x})=\max (\mathbf{x}, 0),
$$

as well as its generalizations.

Given an FNN, we can compute the output from any given input according to $\hat{\mathbf{y}}=\Phi\left(\mathbf{x}^{[0]} ; \Omega\right)$, where $\Omega \triangleq$ 
$\left\{\mathbf{W}^{[n]}, \mathbf{b}^{[n]}, n=0, \ldots, N_{L}+1\right\}$ is the set of parameters of the FNN. Let us denote a labeled training sample as $\left(\mathbf{x}^{[0]}, \mathbf{y}\right)$. In the training phase, a supervised deep learning algorithm optimizes the parameters of the FNN to minimize the expectation of the difference between outputs of the FNN and labels, i.e.,

$$
\min \mathbb{E}_{\mathbf{x}^{[0]}}(\hat{\mathbf{y}}-\mathbf{y})^{2}
$$

where the expectation is taken over input data, $\mathbf{x}^{[0]}$.

2) Universal Approximation Theorem: In wireless networks, an optimal policy maps the state of the system $x^{[0]}$ into the optimal action: $\mathbf{u}^{*}\left(\mathbf{x}^{[0]}\right)$. If we know some optimal state-action pairs, but do not have the expression of $\mathbf{u}^{*}(\cdot)$, then we can use an FNN, $\Phi(\cdot ; \Omega)$, to approximate the policy. The following theorem shows how accurate the FNN can be [66, 223].

Theorem 1. Universal Approximation Theorem: Let $\mathbf{u}^{*}(\cdot)$ be a deterministic continuous function defined over a compact set $\mathcal{D}$, e.g., $\mathbf{x}^{[0]} \in \mathbb{R}^{K}$. Then, for any $\varepsilon_{\mathrm{NN}}>0$, there exists an FNN $\Phi(\cdot ; \Omega)$ constructed by sigmoid activations in $(8)$, such that

$$
\sup _{\mathbf{x}^{[0]} \in \mathcal{D}}\left\|\Phi\left(\mathbf{x}^{[0]} ; \Omega\right)-\mathbf{u}^{*}\left(\mathbf{x}^{[0]}\right)\right\| \leq \varepsilon_{\mathrm{NN}} .
$$

The above theorem has been extended to other kinds of activation functions, including the ReLU function in (9) and its variants [227]. More recently, the results in [223] showed that to achieve a target accuracy, the size of network scales with the approximation accuracy $\varepsilon_{\mathrm{NN}}$ according to $\mathcal{O}\left(\ln \left(1 / \varepsilon_{\mathrm{NN}}\right)\right)$.

\section{B. Predictions in URLLC}

As shown in Table VI supervised deep learning has been employed for channel prediction, mobility prediction, traffic prediction, and QoS or QoE prediction. The reason why supervised deep learning algorithms are very powerful in prediction is that it is very convenient to collect a large number of labeled training samples from the history data, such as trajectories [46, 219], channel and traffic variations [192,211], and QoS experienced by users [220].

1) Channel Prediction: Unreliable wireless channels lead to a high packet loss probability in URLLC [16]. If the transmitter can predict which subchannel will be in deep fading, then packet losses over deep fading channels can be avoided.

By exploiting the temporal correlation of wireless channels, different RNN variants were developed in [211] to predict future signal strength variations. Wideband channels are correlated in the frequency domain. Based on this feature, CNN was used to predict channels for mmWave massive MIMO systems [209]. Although these algorithms are data-driven, the understanding of wireless channel models plays an important role. For example, the structures of neural networks depend on the features of wireless channels (e.g., correlations in temporal and frequency domains). To validate whether the learning algorithms work or not, the model-based methods serve as benchmarks for performance comparison.

2) Traffic Prediction: As shown in [212, 213, 215], predicting the traffic load in a wireless network is helpful for network deployment. These works mainly focus on traffic prediction in a large area. However, to improve the latency, reliability, and resource utilization efficiency of URLLC, the system needs to predict the traffic state of each user. Per-user traffic prediction was studied in [214], the results indicate that LSTM outperforms statistical learning for both short and longterm future predictions. To improve the bandwidth utilization efficiency subject to the latency and reliability requirements, both model-based and data-driven schemes were proposed in [192]. Since URLLC has a stringent requirement on reliability, prediction errors cannot be ignored. With the model-based scheme, the authors of [192] analyzed the prediction error probability in the traffic state prediction. With the datadriven scheme, a better bandwidth utilization efficiency can be achieved, but the prediction error probability can only be evaluated via experiments [192].

3) Mobility Prediction: To reveal the potential gain of mobility prediction in URLLC, a model-based method is applied in the prediction and communication co-design framework in [46]. By predicting the motion of the head and body with LSTM, a predictive pre-rendering approach was proposed in [219]. The results in [46] and [219] indicate that mobility prediction can help reduce user-experienced latency in remote control and VR applications.

It is worth noting that prediction is not error-free. The accuracies of different algorithms in trajectory and location predictions were evaluated in [216] and [217]. Compared with model-based methods, data-driven methods can achieve better prediction accuracies. On the other hand, deriving the performance achieved by data-driven methods is very difficult. To obtain some insights on fundamental trade-offs, such as the latency and reliability trade-off, we still need the help of models [46].

4) QoS Prediction: If a control plane can predict the data plane QoS, it can update resource allocation to avoid QoS violation. For example, by predicting the delay caused by baseband signal processing, a better offloading scheme in could-RAN or MEC systems was developed in [222]. According to the evaluation in [220, 221], supervised deep learning outperforms other baselines in predicting QoE for video services and QoS of IoT applications. Nevertheless, how to predict the QoS or QoE of URLLC services and how to optimize resource allocation for URLLC according to the predicted information remain unclear.

\section{Approximating Optimal Policies}

With the model-based cross-layer optimization in Section $\nabla$ we can obtain optimal policies in wireless networks but with high computing complexity. As a result, these policies can hardly be implemented in real time. To handle this issue, FNNs were used to approximate optimal policies in the existing literature, such as [12,223].

1) Function Approximator: To apply supervised deep learning in URLLC, the analytical tools and optimization algorithms discussed in Sections [V and $\mathrm{V}$ can be used to find labeled samples. Recently, the results in [223] showed that an FNN can be used to approximate an iterative algorithm, 
such as WMMSE for power control. Based on the Universal Approximation Theorem, it is possible to achieve high approximating accuracy. Inspired by these theoretical results, the authors of [224] further showed that by using a CNN model, the required number of weights to achieve the same performance is much smaller than that in an FNN model. DNNs can be also applied to approximate other optimal policies such as bandwidth allocation and user association [11,225].

2) Deep Transfer Learning: To implement supervised deep learning in dynamic wireless networks, the DNN needs to adapt to non-stationary environments and diverse QoS requirements with a few training samples [10]. To achieve this, network-based deep transfer learning is an optional approach [214]. To illustrate the concept of deep transfer learning, we consider two FNNs, $\Phi_{0}\left(\cdot ; \Omega_{0}\right)$ and $\Phi_{1}\left(\cdot ; \Omega_{1}\right)$. The first FNN is well-trained on a data set with a large number of labeled samples, $\mathcal{S}_{0}=\left\{\left(\mathbf{x}_{n_{t}}^{[0]}, \mathbf{y}_{n_{t}}\right), n_{t}=1,2, \ldots, N_{\mathrm{tr}}\right\}$. Now we train a new FNN, $\Phi_{1}\left(\cdot ; \Omega_{1}\right)$, from another data set $\mathcal{S}_{1}=\left\{\left(\tilde{\mathbf{x}}_{n_{f}}^{[0]}, \tilde{\mathbf{y}}_{n_{f}}\right), n_{t}=1,2, \ldots, N_{\mathrm{tf}}\right\}$, where the number of training samples in $\mathcal{S}_{1}$ is much smaller than that in $\mathcal{S}_{0}$, i.e., $N_{\mathrm{tf}} \ll N_{\mathrm{tr}}$. Since the number of labeled samples in $\mathcal{S}_{1}$ is small, we can hardly train $\Phi_{1}\left(\cdot ; \Omega_{1}\right)$ from randomly initialized parameters. If the data sets $\mathcal{S}_{0}$ and $\mathcal{S}_{1}$ are related to each other, then we can initialize the new FNN with $\Omega_{0}$ and update it by using new labeled samples in $\mathcal{S}_{1}$.
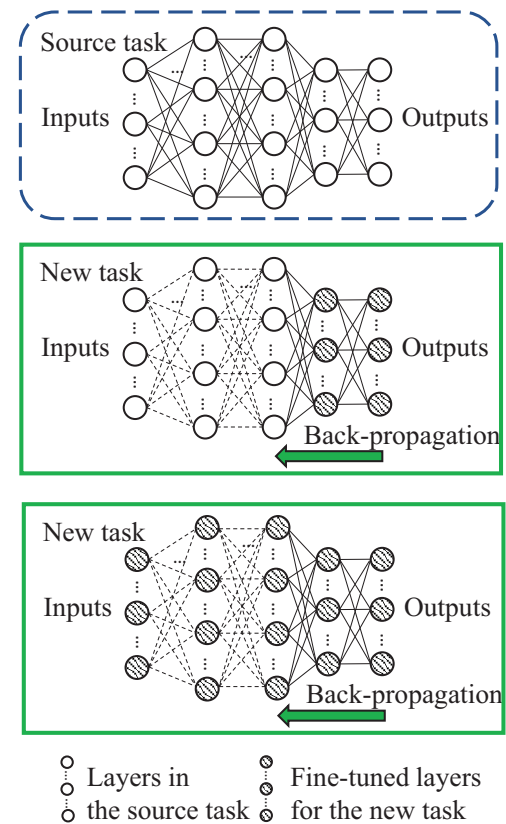

Fig. 10. Illustration of network-based deep transfer learning [10 12]

As illustrated in Fig. 10, $\Omega_{1}$ in the new FNN is initialized with $\Omega_{0}$. In the training phase, the first three layers of $\Omega_{1}$ remain constant, and the back-propagation stops in the fourth layer. Then, fine-tuning is used to refine the weights and bias of the last two layers on the new training data set with small learning rates [228]. Alternatively, we can fine-tune all the parameters of the FNN. The results in [12] show that the latter approach requires more training samples and longer training time, but does not bring significant performance gain.
To determine how many layers should be updated, we need to try different options and find the best one by trial-and-error [12].

\section{Summary of Supervised Deep Learning}

With existing optimization algorithms, a large number of labeled training samples can be obtained off-line for supervised deep learning. After the off-line training phase, the system further fine-tunes DNNs in non-stationary networks. In this way, domain knowledge help to initialize DNNs before online implementation. Deep transfer learning handles the modelmismatch problem with the help of few training samples in new environments.

Although supervised deep learning shows great potential in prediction and function approximation, there are some open problems when using it in URLLC:

- The prediction or approximation accuracy of DNNs will have impacts on the QoS of URLLC. The relative errors achieved by most of the existing supervised learning algorithms in Table VI is around 0.01, which is good enough for traditional services, but far from the requirement of URLLC.

- In some NP-hard problems, labeled training samples are unavailable. For these problems, supervised deep learning is not applicable.

- The number of users and the types of services in a wireless network are dynamic. When features and the dimension of the input data change, deep transfer learning cannot be applied directly.

In the coming two sections, we will review unsupervised deep learning and DRL that do not need labeled training samples.

\section{UNSUPERVISED DEEP LEARNING FOR URLLC}

Unlike supervised deep learning, unsupervised deep learning can find the optimal policy without labeled training samples. In this subsection, we review two branches of existing works that applied unsupervised deep learning in wireless communications, 1) autoencoder for physical-layer communications and 2) FNN trained without labels for resource allocation. The existing works are summarized in Table VII, where the basic idea is to formulate a functional optimization problem and then approximate the optimal function with a DNN trained toward a loss function that represents the goal of the problem .

\section{A. Introduction of Functional Optimization}

A lot of problems in communications can be described by a mapping from an input message (or system state), s, to an output signal (or action), $\mathbf{a}^{\text {s }}$, i.e.,

$$
\mathbf{a}^{\mathrm{s}}=\mathbf{u}(\mathbf{s}) \text {. }
$$

For example, a modulation and coding scheme maps the input bits stream to coding blocks. A scheduler maps QSI and CSI to resource blocks allocated to different users. 
TABLE VII

UNSUPERVISED DEEP LEARNING IN WIRELESS NETWORKS

\begin{tabular}{|c|c|c|c|}
\hline \multicolumn{4}{|c|}{ Part I: Autoencoder for Physical-layer Communications (Unconstrained Functional Optimization) } \\
\hline Year & Reference & Summary & Performance \\
\hline 2018 & 61 & An autoencoder was used to learn a better constellation & $\begin{array}{l}\text { The BLER gap between fine-tuned Autoencoder and } \\
\text { an existing constellation is } 1 \mathrm{~dB}\end{array}$ \\
\hline 2018 & 229 & $\begin{array}{l}\text { An autoencoder was applied for E2E learning in OFDM systems } \\
\text { for reliable communications over multipath channels }\end{array}$ & $\begin{array}{l}\text { Autoencoder achieves better BLER than Quadrature } \\
\text { Phase Shift Keying (QPSK) }\end{array}$ \\
\hline 2018 & [230] & $\begin{array}{l}\text { A joint source and channel coding scheme was proposed to } \\
\text { minimize the E2E distortion }\end{array}$ & $\begin{array}{l}\text { The learning-based method outperforms an existing } \\
\text { separate source and channel coding scheme }\end{array}$ \\
\hline 2019 & [231] & $\begin{array}{l}\text { An autoencoder was used for multiuser waveform design at the } \\
\text { transmitter side }\end{array}$ & $\begin{array}{l}\text { The learning-based method outperforms linear } \\
\text { MMSE in terms of lower BLER }\end{array}$ \\
\hline \multicolumn{4}{|c|}{ Part II: Primal-dual Method for Radio Resource Allocation (Constrained Functional Optimization) } \\
\hline Year & Reference & Summary & Performance \\
\hline 2019 & 63] & $\begin{array}{l}\text { The primal-dual method is used to train an FNN to find the optimal } \\
\text { resource allocation subject to statistic constraints }\end{array}$ & $\begin{array}{l}\text { The sum-capacity of the learning-based method is } \\
\text { close to WMMSE }\end{array}$ \\
\hline 2019 & 121 & $\begin{array}{l}\text { Applying the primal-dual method to train DNNs for distributed } \\
\text { wireless resource management problems }\end{array}$ & $\begin{array}{l}\text { Distributed DNNs can achieve near-optimal sum- } \\
\text { capacity }\end{array}$ \\
\hline 2019 & [26] & $\begin{array}{l}\text { Applying the primal-dual method to train a DNN for resource } \\
\text { allocation subject to the QoS constraints of URLLC }\end{array}$ & $\begin{array}{l}\text { The QoS of URLLC can be satisfied by reserving } \\
1 \% \text { of extra bandwidth }\end{array}$ \\
\hline 2019 & [232] & $\begin{array}{l}\text { Developing a model-free primal-dual algorithm for learning opti- } \\
\text { mal ergodic resource allocations }\end{array}$ & $\begin{array}{l}\text { The sum-capacity achieved by the model-free ap- } \\
\text { proach is close to WMMSE }\end{array}$ \\
\hline 2020 & 233] & $\begin{array}{l}\text { Developing a reinforced-unsupervised learning framework for the } \\
\text { model-free optimization problems }\end{array}$ & $\begin{array}{l}\text { Unsupervised learning outperforms supervised learn- } \\
\text { ing when the number of training samples is large }\end{array}$ \\
\hline 2020 & 234 & $\begin{array}{l}\text { Training a Graph Neural Network (GNN) to find the optimal } \\
\text { resource allocation in large-scale wireless networks }\end{array}$ & GNN outperforms WMMSE in large-scale networks \\
\hline
\end{tabular}

1) Variable Optimization Problems: A regular variable optimization problem can be formulated in a general form as follows,

$$
\begin{aligned}
& \min _{\mathbf{a}^{\mathrm{s}}} F\left(\mathbf{s}, \mathbf{a}^{\mathrm{s}}\right) \\
& \text { s.t. } G_{j}\left(\mathbf{s}, \mathbf{a}^{\mathrm{s}}\right) \leq 0, j=1, \ldots, J,
\end{aligned}
$$

where $F\left(\mathbf{s}, \mathbf{a}^{\mathrm{s}}\right)$ is the objective function, 111a) are the constraints on available resources or QoS.

By solving problem (11), we can obtain the optimal solution $\mathbf{a}^{\mathrm{s} *}$ for a given input message or system state $\mathbf{s}$. When the input message or the state varies, the system needs to update $\mathbf{a}^{\mathrm{s} *}$ by solving problem (11) numerically, unless the optimal solution can be derived in a closed-form expression with respect to s. However, in most of the cases, we can hardly derive the closed-form expression, and optimization algorithms for solving problem (11) are too complicated to be implemented in real-time.

2) Functional Optimization Problems: As proved in [26], variable optimization problem (11) is equivalent to the following functional optimization problem,

$$
\begin{aligned}
\min _{\mathbf{u}(\cdot)} & \mathbb{E}_{\mathbf{s}}[F(\mathbf{s}, \mathbf{u}(\mathbf{s}))] \\
\text { s.t. } & G_{j}(\mathbf{s}, \mathbf{u}(\mathbf{s})) \leq 0, \forall \mathbf{s} \in \mathcal{D}_{\mathrm{s}}, j=1, \ldots, J .
\end{aligned}
$$

In problem (12), the elements to be optimized are resource allocation or scheduler functions with respect to the states of users. By solving the above problem, we can obtain the expression of the optimal policy. However, solving a functional optimization problem is more challenging than solving variable optimization problems [43,235]. This is because the optimization variables are functions, each of which is equiva- lent to an infinite-dimensional vector (e.g., Fourier series with infinite terms). Moreover, each constraint in (12a) is equivalent to an infinite number of constraints in variable optimization problems (constraints for any given value of $\mathbf{s}$ ).

3) Methods for Solving Functional Optimization Problems: To derive the closed-form expression of a functional optimization problem, we can find the solutions that satisfy the firstorder necessary conditions in [236]. For example, optimizing the instantaneous power control policy to maximize the ergodic capacity under the maximal transmit power constraint belongs to functional optimization problems [24]. By solving the first-order necessary conditions, we can derive that the optimal power control policy is the water-filling policy. Compared with this physical-layer optimization problem, crosslayer optimization problems are much more complicated and the closed-form expressions of optimal policies are usually unavailable. For example, C. She et al. investigated how to adjust transmit power and subcarrier allocation according to QSI in MIMO-Orthogonal Frequency Division Multiplexing (OFDM) systems. The problem was formulated as a functional optimization problem, and a closed-form solution can only be obtained for a specific packet arrival process in an asymptotic case: the number of antennas at the BS goes to infinite [167].

When there is no closed-form solution, we need numerical methods to find near-optimal solutions. There are several numerical methods for solving functional optimization problems, such as the finite element method (FEM) [237]. The basic idea of the FEM is to parameterize $\mathbf{u}(\mathbf{s})$ with a finite sampled points, and approximate the optimal solution by optimizing the values on the sampled points. However, the number of the sampled points increases exponentially with the dimension of $\mathbf{s}$. In addition, these numerical methods are usually used 
to solve problems formulated in the three-dimensional space, and heavily rely on the theorems in dynamics [238]. Whether they can work well with high-dimensional communication problems remains unclear.

Inspired by the Universal Approximation Theorem in 66. 227], we can use a DNN to approximate the optimal policy $\mathbf{u}^{*}(\cdot)$ that maps the state $\mathbf{s}$ to the optimal solution $\mathbf{a}^{\mathbf{s}}$ and find the parameters of the DNN with unsupervised deep learning methods.

\section{B. Autoencoder for E2E Design in Physical-Layer Communi- cations}

Autoencoder is a useful tool for data compression and feature extraction [239]. The basic idea is to train DNNs with the input data. An autoencoder consists of three components: an encoder, a decoder, and a distortion function that compares the input and the output of the autoencoder.

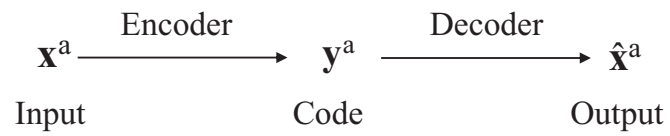

(a) Autoencoder

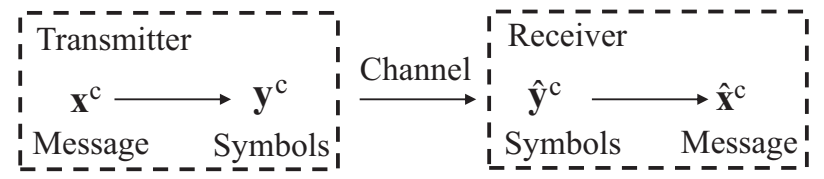

(b) Physical-layer communications

Fig. 11. Autoencoder for physical-layer communications 61,239].

As shown in Fig. 11 (a), the input and the output of the autoencoder are $\mathrm{x}^{\mathrm{a}} \in \mathbb{R}^{N_{\mathrm{x}}^{\mathrm{a}} \times 1}$ and $\hat{\mathrm{x}}^{\mathrm{a}} \in \mathbb{R}^{N_{\mathrm{x}}^{\mathrm{a}} \times 1}$, respectively. The compressed code is $\mathrm{y}^{\mathrm{a}} \in \mathbb{R}^{N_{\mathrm{y}}^{\mathrm{a}} \times 1}$, where $N_{\mathrm{y}}^{\mathrm{a}}<N_{\mathrm{x}}^{\mathrm{a}}$. Denote the encoder and the decoder by $f_{\mathrm{e}}(\cdot)$ and $f_{\mathrm{d}}(\cdot)$, respectively. The autoencoder problem is to find $f_{\mathrm{e}}(\cdot)$ and $f_{\mathrm{d}}(\cdot)$ that minimize the average of the distortion function [239],

$$
\min _{f_{\mathrm{e}}(\cdot), f_{\mathrm{d}}(\cdot)} \mathbb{E}\left(\hat{\mathbf{x}}^{\mathrm{a}}-\mathbf{x}^{\mathrm{a}}\right)^{2}
$$

where the average is taken over the input, and $\left(\hat{\mathbf{x}}^{\mathrm{a}}-\mathrm{x}^{\mathrm{a}}\right)^{2}$ is the distortion function. Problem (13) is an unconstrained functional optimization problem [235]. To solve the functional optimization problem, a widely applied approach is approximating the encoder and the decoder with two DNNs [240].

The components of physical-layer communications are shown in Fig. 11 b), which are very similar to that of an autoencoder. The transmitter sends message $\mathrm{x}^{\mathrm{c}}$ to the receiver over a certain channel. First, the transmitter encodes the message into a coding block that consists of multiple symbols, $\mathbf{y}^{\mathrm{c}}$, such as $M$-QAM symbols. Then, the block is sent over a channel. Owning to the noise and interference, the received symbols, denoted by $\hat{\mathbf{y}}^{\mathrm{c}}$, are different from $\mathbf{y}^{\mathrm{c}}$. Based on the received symbols, the receiver tries to decode the block and recover the message $\hat{\mathbf{x}}^{\mathrm{c}}$. A communication problem is to find the optimal encoder and decoder that minimize the BLER, i.e.,

$$
\min _{f_{\mathrm{e}}(\cdot), f_{\mathrm{d}}(\cdot)} \mathbb{E}\left\{\mathbf{1}\left(\hat{\mathbf{x}}^{\mathrm{a}} \neq \mathbf{x}^{\mathrm{a}}\right)\right\}
$$

where the average is taken over input messages and stochastic channels, and $\mathbf{1}\left(\hat{\mathbf{x}}^{\mathrm{a}} \neq \mathrm{x}^{\mathrm{a}}\right)$ is an indicator of event $\hat{\mathbf{x}}^{\mathrm{a}} \neq \mathrm{x}^{\mathrm{a}}$. If $\hat{\mathbf{x}}^{\mathrm{a}} \neq \mathrm{x}^{\mathrm{a}}$ happens, then the indicator equals to 1 . Otherwise, it equals to 0 .

The encoder and the decoder of the communication system can be approximated by two DNNs [61,230]. To allow for back-propagation, a channel should be approximated by a DNN [230]. Considering that the real-world channel is not available, the autoencoder is first trained with a stochastic channel model and then implemented in real hardware for over-the-air transmissions [61]. To compensate for the mismatch between the model and the real-world channel, the system can fine-tune the receiver part of the autoencoder.

As summarized in Part I of Table VII the above method has been implemented in several communication systems. Since the goal is to minimize the difference between the input data and output data, the autoencoder is suitable for URLLC. For example, the results in [231] show that the scheme obtained with the autoencoder can achieve much lower BLER than the linear minimum mean-square error MIMO receiver. In addition, autoencoders inherently learn how to deal with hardware impairments [61, 229]. In other words, they do not rely on the accuracy of models in communication systems. Furthermore, after the training phase, the outputs of the encoder and the decoder, represented by DNNs, can be computed from the forward propagation algorithm, which has much lower complexity than existing modulation and coding schemes.

\section{Optimizing Resource Allocation}

1) Model-based learning: In the case that the objective function and constraints can be derived with the model-based method in Section IV] a resource allocation problem can be formulated in a general form as follows,

$$
\begin{aligned}
\min _{\mathbf{u}(\cdot)} & \mathbb{E}_{\mathbf{s}}[F(\mathbf{s}, \mathbf{u}(\mathbf{s}))] \\
\text { s.t. } & \mathbb{E}_{\mathbf{s}}\left[G_{i}^{\text {ave }}(\mathbf{s}, \mathbf{u}(\mathbf{s}))\right] \leq 0, i=1, \ldots, I, \\
& G_{j}^{\text {ins }}(\mathbf{s}, \mathbf{u}(\mathbf{s})) \leq 0, \forall \mathbf{s} \in \mathcal{D}_{\mathrm{s}}, j=1, \ldots, J, \\
& \mathbf{u}(\mathbf{s}) \in \mathcal{D}_{\mathrm{a}} .
\end{aligned}
$$

In addition to the instantaneous constraints in problem (11), long-term statistic constraints in 14a are considered. The statistic constraints can be the average transmit power constraint [24] or the statistical QoS constraint [172]. The instantaneous constraints ensure instantaneous requirements, such as the maximal transmit power constraint. Here, the objective function and the constraints are differentiable but not necessary to be convex.

The primal-dual method for solving functional optimization problems has been extensively used in optimal control theory [241]. To apply the method, we first define the Lagrangian of problem (14) in Fig. 12, where the Lagrange multipliers, $\lambda_{i}^{\mathrm{G}} \geq 0$ and $v_{j}^{\mathrm{G}}(\mathbf{s}) \geq 0, \forall \mathbf{s} \in D_{\mathrm{s}}$, are the weighing coefficients of the constraints. To solve problem (14), we turn to solve the 


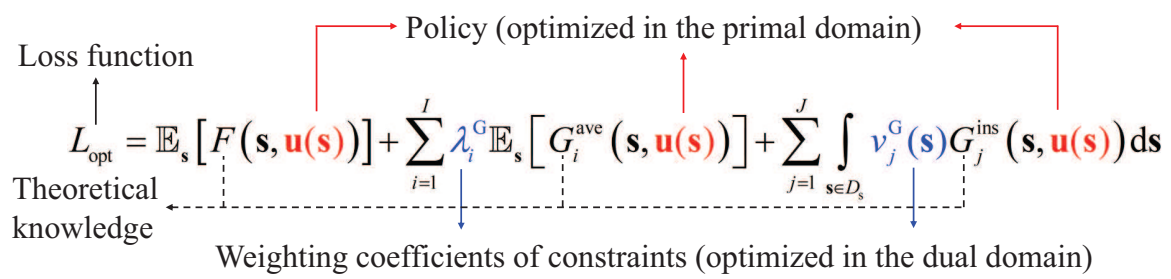

Fig. 12. Definition of Lagrangian [67].

following problem:

$$
\begin{aligned}
\max _{\substack{\lambda_{i}^{\mathrm{G}}, i=1, \ldots, I \\
v_{j}^{\mathrm{G}}(\mathbf{s}), j=1, \ldots, J}} & \min _{\mathbf{u}(\mathbf{s})} L_{\mathrm{opt}}, \\
& \text { s.t. } \lambda_{i}^{\mathrm{G}} \geq 0, v_{j}^{\mathrm{G}}(\mathbf{s}) \geq 0, \forall \mathbf{s} \in D_{\text {s. }} .
\end{aligned}
$$

where the policy $\mathbf{u}(\mathbf{s})$ and the Lagrangian multipliers are optimized in the primal and dual domains, iteratively.

To obtain $\mathbf{u}(\mathbf{s})$ and Lagrange multipliers numerically, two neural networks, $\Phi^{\mathrm{u}}\left(\mathbf{s} ; \Omega^{\mathrm{u}}\right)$ and $\Phi^{\mathrm{v}}\left(\mathbf{s} ; \Omega^{\mathrm{v}}\right)$, are used to approximate $\mathbf{u}(\mathbf{s})$ and $\mathbf{v}^{\mathrm{G}}(\mathbf{s})$, respectively, where $\mathbf{v}^{\mathrm{G}}(\mathbf{s}) \triangleq$ $\left[v_{1}^{\mathrm{G}}(\mathbf{s}), \ldots, v_{J}^{\mathrm{G}}(\mathbf{s})\right]^{T}$. Then, by using the Lagrangian in Fig. 12 as the loss function, we can update between primal variables $\Omega^{\mathrm{u}}$, and dual variables $\lambda_{i}^{\mathrm{G}}$ and $\Omega^{\mathrm{v}}$ iteratively with the stochastic gradient descent algorithm. As will be shown in the case studies, by integrating the theoretical formulations of objective function and constraints into the loss function, unsupervised deep learning outperforms supervised deep learning in terms of approximation accuracy.

For the applications in Table I] communication systems need to satisfy multiple conflicting KPIs, which are either considered in the constraints or formulated as the objective function. A straightforward approach is to minimize/maximize a weighted sum of different KPIs by setting the weighting coefficients manually. From the definition of Lagrangian in Fig. 12, we can see that with different weighting coefficients, the optimal policies that minimize the Lagrangian are different. The primal-dual method is able to find the optimal weighting coefficients achieving the target balance between the objective function and constraints. As such, the objective function is minimized and the constraints are satisfied [121].

2) Model-free learning: In the case that there is no theoretical models of the considered system, the expressions of $F, G_{i}^{\text {ave }}$, and $G_{j}^{\text {ins }}$ and the gradients or derivatives of these functions are unavailable. For an optimization problem as in (14), one can train neural networks to approximate these functions according to the observed values of these functions with the supervised deep learning method. Then, the unknown gradients or derivatives can be estimated by the neural networks through backward propagation. The details on the model-free unsupervised deep learning method can be found in $[63,232,233,242]$.

\section{Summary of Unsupervised Deep Learning}

Theoretical models can be used to initialize autoencoders and formulate optimization problems. Since autoencoders are trained from the input data, they do not require analytical models of communication systems and can handle hardware im- pairment. For analytically intractable optimization problems, an approximation of the optimal solution can be obtained by combining the formulas into the loss function of unsupervised deep learning, i.e., Lagrangian.

Although unsupervised deep learning shows above advantages over supervised deep learning, it also suffers from some critical issues.

- When applying autoencoders in physical-layer design, the function that maps the input to the output of real-world channels is not available, and hence we cannot obtain the gradient required to fine-tune the transmitter.

- When applying model-based unsupervised deep learning in functional optimization, the objective function and constraints should be differentiable. Thus, it cannot be applied to integer or mixed-integer programming directly.

- Like supervised deep learning, how to update the neural network according to dynamic features, such as the dimension of the input data, needs further investigation.

In the next section, we will illustrate DRL that can optimize both continuous and discrete variables.

\section{DEEP REINFORCEMENT LEARNING FOR URLLC}

DRL was widely applied in optimal control of stochastic processes. The most important feature of DRL is that it learns from the feedback that evaluates the actions taken rather than learns from correct actions. In this section, we first introduce the basics in DRL and then summarize the existing studies that applied DRL in wireless networks. To guarantee the QoS requirements in URLLC, we review a promising candidate: constrained DRL. Finally, we illustrate how to combine modelbased tools with DRL in URLLC.

\section{A. Introduction of DRL}

1) Elements of Reinforcement Learning: In reinforcement learning, there is an agent (or multiple agents) that observes the reward signal from the environment, takes actions according to a policy, and tries to maximize a value function. If a model of the environment is available, it can help improve learning efficiency.

Policy: Let $\mathbf{s}(t) \in \mathcal{D}_{\mathrm{s}}$ and $\mathbf{a}^{\mathrm{s}}(t) \in \mathcal{D}_{\mathrm{a}}$ denote the state and the action in the $t$-th time slot. A policy is a mapping from states of the environment to actions an agent may take when in those states. We denote a stochastic policy by $\pi$. When the numbers of states and actions are finite, $\pi$ consists of the probabilities to perform different actions in every state.

Reward signal: In each time slot, a real number, the reward signal, is sent to the agent from the environment. It defines 
the goal of the problem, and the agent aims to maximize the long term reward. The reward signal depends on the states and actions taken by the agent, but the agent cannot change the function that generates the reward signal. We denote the reward signal in the $t$-th slot by $r\left(\mathbf{s}(t), \mathbf{a}^{\mathbf{s}}(t)\right)$, which can be simplified as $r(t)$.

Value functions: In general, a reinforcement learning algorithm aims to maximize the long-term return, such as the accumulated discounted reward,

$$
G(t)=\sum_{i=t}^{\infty} \gamma_{\mathrm{d}}^{i-t} r\left(\mathbf{s}(i), \mathbf{a}^{\mathrm{s}}(i)\right),
$$

where $\gamma_{\mathrm{d}} \in[0,1)$ is the discounting factor. $G(t)$ reflects the long-term return that the agent will receive after the $t$-th slot in an infinite horizon. Similarly, we can also formulate the discounted reward in a finite horizon if the task ends after finite steps, $G(t)=\sum_{i=t}^{t_{\max }} \gamma_{\mathrm{d}}^{i-t} r\left(\mathbf{s}(i), \mathbf{a}^{\mathrm{s}}(i)\right)$. This definition is also referred to as partial return in [68]. The value of taking action $\mathbf{a}^{\mathbf{s}}(t)$ in state $\mathbf{s}(t)$ under a policy $\pi$, denoted by $Q^{\pi}\left(\mathbf{s}(t), \mathbf{a}^{\mathrm{s}}(t)\right)$, is the expected return starting from state $\mathbf{s}(t)$, taking the action $\mathbf{a}^{\mathbf{s}}(t)$, and thereafter following policy $\pi$. $Q^{\pi}\left(\mathbf{s}(t), \mathbf{a}^{\mathrm{s}}(t)\right)$ is also known as the state-action value function. Similarly, the expected return of visiting state $\mathbf{s}(t)$ is defined as $V^{\pi}(\mathbf{s}(t))$, which is referred to as the state value function.

2) Exploitation and Exploration: The trade-off between exploitation and exploration is one of the challenges that raise in reinforcement learning. By exploiting the experience, the agent can obtain a good reward that maximizes the estimated value function. To explore better actions, the agent needs to try some actions that are not optimal according to the previous experience. There are many sophisticated methods to balance exploitation and exploration, such as $\varepsilon$-greedy methods [243], using optimistic initial values [68], and the Bayesian sampling approach [244]. However, when using reinforcement learning in URLLC, we need to satisfy the QoS constraints during explorations. Thus, random explorations are not applicable. We will discuss how to re-design exploration schemes for URLLC in Section VIII-E.

3) Markov Decision Process (MDP): To apply reinforcement learning in optimal control of a dynamic system, the uncertainty of the system should be formulated in the framework of MDP [68]. An MDP is a controlled stochastic process defined by a state space, $\mathcal{D}_{\mathrm{s}}$, an action space, $\mathcal{D}_{\mathrm{a}}$, a reward function, $\mathcal{D}_{\mathrm{s}} \times \mathcal{D}_{\mathrm{a}} \times \mathcal{D}_{\mathrm{s}} \rightarrow \mathbb{R}$, that generates rewards signal according to the states and the action, and transition probabilities, i.e.,

$$
\begin{aligned}
& \operatorname{Pr}[\mathbf{s}(t+1), r(t+1) \mid \mathbf{s}(t), \mathbf{a}(t)] \\
& =\operatorname{Pr}[\mathbf{s}(t+1), r(t+1) \mid \mathbf{s}(0), \mathbf{a}(0), r(0), \ldots, \mathbf{s}(t), \mathbf{a}(t), r(t)] .
\end{aligned}
$$

The transition probabilities in (17) indicate that we can predict the state and reward in the $(t+1)$-th slot based solely on the state and action in the $t$-th time slot just as well as we know the states and actions in all the previous slots. Such a property is referred to as Markov property or "memorylessness".

In reinforcement learning, the transition probabilities of the
MDP are referred to as the "model" of the problem. This "model" is definitely different from the models in communications and networking. Depending on whether the transition probabilities are available or not, reinforcement learning algorithms are classified into two categories: model-based and model-free [23, 68]. In this work, we only review the modelfree reinforcement learning that does not need the transition probabilities, and use the term knowledge-assisted reinforcement learning to represent another category of algorithms that exploit domain knowledge to improve the learning efficiency.

4) Bellman Equation: In the framework of MDP, $Q^{\pi}\left(\mathbf{s}(t), \mathbf{a}^{\mathrm{s}}(t)\right)$ can be formally defined as follows,

$$
Q^{\pi}\left(\mathbf{s}(t), \mathbf{a}^{\mathbf{s}}(t)\right)=\mathbb{E}\left[G(t) \mid \mathbf{s}(t), \mathbf{a}^{\mathbf{s}}(t)\right] .
$$

and the expectation in (18) is taken over $\left\{r\left(\mathbf{s}(i), \mathbf{a}^{\mathrm{s}}(i)\right), \mathbf{s}(i+1), \mathbf{a}^{\mathrm{s}}(i+1), i \geq t\right\}$, following policy $\pi$. We call the function in (18) the action-value function for policy $\pi$.

By applying $G(t)=r\left(\mathbf{s}(t), \mathbf{a}^{\mathbf{s}}(t)\right)+G(t+1)$, we can express the value function in recursive form, known as the Bellman equation [68]. To avoid complicated expectations, we illustrate the Bellman equation with a deterministic policy, which can be described as $\mathbf{a}^{\mathrm{s}}(t)=\mathbf{u}(\mathbf{s}(t))$. In this case, the value function in (18) can be simplified as follows,

$$
\begin{aligned}
& Q^{\mathbf{u}}\left(\mathbf{s}(t), \mathbf{a}^{\mathbf{s}}(t)\right) \\
& =\mathbb{E}\left[r\left(\mathbf{s}(t), \mathbf{a}^{\mathbf{s}}(t)\right)+\gamma_{\mathrm{d}} Q^{\mathbf{u}}(\mathbf{s}(t+1), \mathbf{u}(\mathbf{s}(t+1)))\right],
\end{aligned}
$$

where the expectation is taken over $r\left(\mathbf{s}(t), \mathbf{a}^{\mathbf{s}}(t)\right)$ and $\mathbf{s}(t+1)$.

5) Actor-Critic Algorithm: For a reinforcement learning problem, we aim to find the optimal policy that maximizes the value function in (19). It is well known that when the transition probabilities are known at the agent, then the optimal policy can be obtained by dynamic programming [245]. However, due to the curse of dimensionality, dynamic programming can hardly be implemented when the dimensions of observation/action spaces are high. To handle this issue, the actor-critic algorithm was proposed in [246]. The basic idea of the actor-critic algorithm is combining DNN with reinforcement learning by approximating the policy and the value function with two DNNs [247]. For example, if the policy is a deterministic and continuous function, the deep deterministic policy gradient algorithm can be applied [64].

An actor-critic algorithm with FNNs is illustrated in Fig. 13, Given the state in the current time slot, $\mathbf{s}(t)$, the actor takes action according to the output of the FNN. Then, the reward from the environment is sent to the actor and the critic for updating the policy and the approximation of the value function, respectively. For a deterministic policy, an actorcritic, model-free algorithm, was proposed in [64], where the algorithm directly learn from the real-world environment. To avoid QoS violations during the training phase, the authors of [11] train the policy in a simulation platform that is built upon theoretical models and practical configurations. 
TABLE VIII

DRL IN WIRELESS NETWORKS

\begin{tabular}{|c|c|c|}
\hline \multicolumn{3}{|c|}{ Surveys or Tutorials } \\
\hline Reference & \multicolumn{2}{|l|}{ Contributions } \\
\hline 60 & \multicolumn{2}{|c|}{ A comprehensive survey on applications of DRL in communications and networking } \\
\hline 22 & \multicolumn{2}{|c|}{ A tutorial on the implementation of DRL in beyond 5G systems } \\
\hline 248 & \multicolumn{2}{|c|}{ A tutorial on the applying DRL in resource allocation at the network edge } \\
\hline \multicolumn{3}{|c|}{ Mobile Networking } \\
\hline Reference & Contributions & Performance \\
\hline [249] & $\begin{array}{l}\text { Optimizing handover controller at each user via a model-free } \\
\text { asynchronous advantage actor-critic (A3C) framework }\end{array}$ & $\begin{array}{l}\text { A3C outperforms the upper confidence bandit algorithm and } \\
\text { the } 3 \text { GPP protocol in terms of throughput and handover rate }\end{array}$ \\
\hline [250] & $\begin{array}{l}\text { Mitigating internet congestion by adjusting the data rate of } \\
\text { each sender via DRL }\end{array}$ & $\begin{array}{l}\text { The proposed framework can achieve a better latency- } \\
\text { throughput trade-off than existing approaches }\end{array}$ \\
\hline 251] & $\begin{array}{l}\text { A fast-learning DRL model that dynamically optimizing net- } \\
\text { work slicing in WiFi networks }\end{array}$ & $\begin{array}{l}\text { The proposed model-free approach with pre-training is } \\
\text { promising in dynamic environments }\end{array}$ \\
\hline \multicolumn{3}{|c|}{ Scheduler Design } \\
\hline Reference & Contributions & Performance \\
\hline$[252$ & $\begin{array}{l}\text { Energy-efficient scheduling policy for vehicle safety and QoS } \\
\text { concerns }\end{array}$ & $\begin{array}{l}\text { The DRL approach outperforms other methods in terms of } \\
\text { incomplete requests, battery lifetime, and latency }\end{array}$ \\
\hline 253 & $\begin{array}{l}\text { Energy-efficient user scheduling and resource allocation in } \\
\text { heterogeneous networks }\end{array}$ & $\begin{array}{l}\text { Actor-critic learning can achieve higher reward than Q- } \\
\text { learning }\end{array}$ \\
\hline [254] & $\begin{array}{l}\text { Actor-critic DRL for traffic scheduling in cellular networks } \\
\text { with measured data }\end{array}$ & $\begin{array}{l}\text { DRL method can achieve higher reward (network utilization, } \\
\text { throughput, congestion) than some benchmarks }\end{array}$ \\
\hline 255 & $\begin{array}{l}\text { Scheduler selection for minimizing packet delays and packet } \\
\text { drop rates }\end{array}$ & $\begin{array}{l}\text { The proposed policy outperforms existing scheduling policies } \\
\text { in terms of both delay and packet drop rate }\end{array}$ \\
\hline [256] & $\begin{array}{l}\text { Task scheduling and offloading for minimizing average slow- } \\
\text { down and timeout period in MEC }\end{array}$ & $\begin{array}{l}\text { DRL method outperforms existing schedulers in terms of } \\
\text { average slowdown and average timeout period }\end{array}$ \\
\hline \multicolumn{3}{|c|}{$\begin{array}{l}\text { Resource Allocation } \\
\end{array}$} \\
\hline Reference & Contributions & Performance \\
\hline 257 & $\begin{array}{l}\text { DRL for switching remote radio heads ON and OFF in could } \\
\text { radio access networks }\end{array}$ & $\begin{array}{l}\text { DRL outperforms single BS association and full coordinated } \\
\text { association in terms of power consumption }\end{array}$ \\
\hline 258 & $\begin{array}{l}\text { Minimizing execution delay and energy consumption in MEC } \\
\text { by optimizing offloading and resource allocation }\end{array}$ & $\begin{array}{l}\text { DRL outperforms benchmarks in terms of execution delay } \\
\text { and energy consumption }\end{array}$ \\
\hline 259 & $\begin{array}{l}\text { Decentralized resource allocation for vehicle-to-vehicle com- } \\
\text { munications }\end{array}$ & $\begin{array}{l}\text { DRL achieves higher data rates in vehicle-to-vehicle and } \\
\text { vehicle-to-infrastructure links than existing methods }\end{array}$ \\
\hline [260] & $\begin{array}{l}\text { Maximizing weighted sum of spectrum efficiency and quality- } \\
\text { of-experience in network slicing }\end{array}$ & $\begin{array}{l}\text { DRL can enhance the effectiveness and agility for network } \\
\text { slicing by matching the allocated resource to users' demand }\end{array}$ \\
\hline 261 & $\begin{array}{l}\text { Minimizing long-term system power consumption in Fog- } \\
\text { RAN }\end{array}$ & $\begin{array}{l}\text { The energy consumption achieved by DRL is lower than } \\
\text { benchmarks and can be further reduced by transfer learning }\end{array}$ \\
\hline 262] & $\begin{array}{l}\text { Minimizing average energy consumption or average latency } \\
\text { in MEC IoT networks by using Monte Carlo tree search and } \\
\text { multi-task learning }\end{array}$ & $\begin{array}{l}\text { The proposed DRL framework outperforms other DRL algo- } \\
\text { rithms and benchmarks in terms of average energy consump- } \\
\text { tion and average service latency }\end{array}$ \\
\hline 263 & $\begin{array}{l}\text { Applying the model-based DRL in [23] for resource manage- } \\
\text { ment in network slicing }\end{array}$ & $\begin{array}{l}\text { By introducing discrete normalized advantage functions into } \\
\text { DRL, better SE and QoE can be achieved }\end{array}$ \\
\hline 264 & $\begin{array}{l}\text { Applying DRL in broadcast beam optimization for both } \\
\text { periodic and Markov mobility patterns }\end{array}$ & $\begin{array}{l}\text { DRL converges to the optimal solutions and can adjust actions } \\
\text { according to user distributions }\end{array}$ \\
\hline \multicolumn{3}{|c|}{$\begin{array}{ll} & \text { Routing } \\
\end{array}$} \\
\hline Reference & Contributions & Performance \\
\hline 265.266 & $\begin{array}{l}\text { Developing a GNN-based framework, RouteNet, to predict } \\
\text { mean delay, jitter and packet loss [265] and combining GNN } \\
\text { in DRL for routing optimization [266] }\end{array}$ & $\begin{array}{l}\text { RouteNet outperforms model-based analysis in terms of pre- } \\
\text { diction accuracy and GNN+DRL outperforms existing DRL, } \\
\text { especially when the topology is changing }\end{array}$ \\
\hline
\end{tabular}




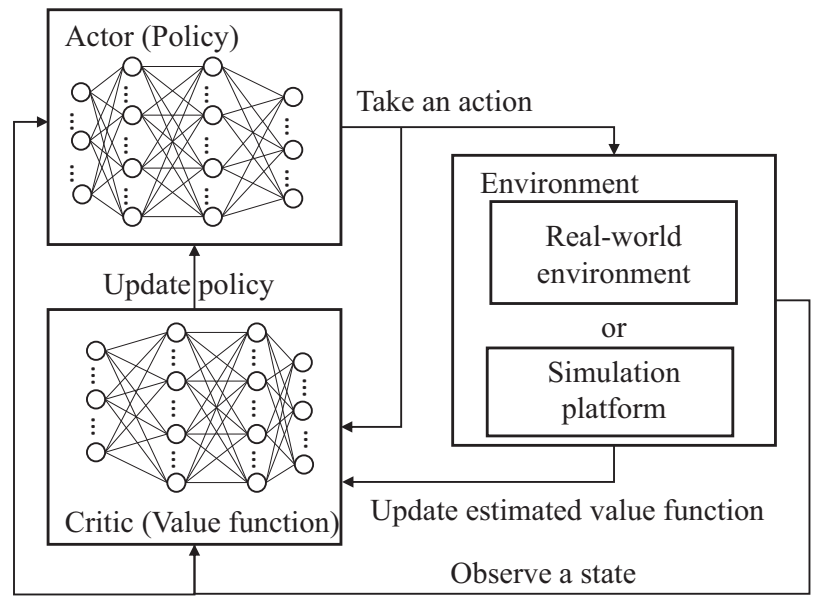

Fig. 13. Illustration of actor-critic algorithm with FNNs [64:68].

\section{B. DRL in Wireless Networks}

As summarized in Table VIII DRL algorithms have been applied in wireless networks for mobile networking [249, 251], scheduler design [252,-256], resource allocation [257, 258 260. 262,264], and routing [265, 266].

Key Challenges: According to [254], research challenges when applying DRL in wireless networks lie in the following aspects,

- Non-Markovian Processes: In practical systems, optimal control problems may not be Markovian. For example, the network dynamics in the real-world scenarios in [254] are not Markovian. However, to implement DRL, we need to formulate the problem in the framework of MDP. Otherwise, the Bellman equation does not hold.

- Safe Exploration: Exploration in the unknown environment may lead to bad feedback, especially for missioncritical IoT in factory automation or vehicle networks. If an action cannot guarantee ultra-high reliability, then it will result in unexpected accidents. Thus, we need to avoid bad actions during explorations to improve the safety of a system.

- Long Convergence Time: With high-dimensional observation/action spaces, the convergence time of model-free DRL will be long. In addition, estimating the reliability of URLLC in real-world networks is time-consuming. For example, to evaluate the reward of an action in URLLC, the system needs to send a large number of packets for estimating the reliability of an action [11]. As a result, it takes a very long time for approximating the value function.

In the following subsections, we review some methods to address the above challenges.

\section{Techniques for Improving DRL in URLLC}

When using DRL, there is no strict proof of the convergence owning to the approximations of the policy and the value function [68]. Empirical evaluations in some existing publications showed that DRL converges slowly when the state and action spaces are large [64 247]. Thus, how to reduce the convergence time of DRL is one of the most important topics in this area [23]. On the other hand, the approximation errors of the policy and value function may have impacts on the reliability of URLLC. How to achieve high reliability when using DRL to optimize communication systems has not been investigated in the existing literature. In this subsection, we review some promising techniques for reducing the convergence time and improving the approximation accuracy of DRL.

1) Reward Shaping: As discussed in [267], delayed reward remains one of the key challenges in many reinforcement learning problems. It means that an agent receives a delayed reward after taking several steps. For example, when playing chess, the agent only receives a positive or negative reward by the end of a game.

Reward shaping is a technique to handle this issue by modifying the reward function according to the design goal of a system [268]. With reward shaping, the instantaneous reward in the $t$-th slot is redefined as

$\tilde{r}\left(\mathbf{s}(t), \mathbf{a}^{\mathrm{s}}(t)\right)=r\left(\mathbf{s}(t), \mathbf{a}^{\mathrm{s}}(t)\right)-\Psi(\mathbf{s}(t))+\gamma_{\mathrm{d}} \Psi(\mathbf{s}(t+1))$,

where $\Psi(\mathbf{s}(t))$ is referred to as the potential function in [268]. It is a function that depends on the state and does not rely on the action. Such a function reflects the potential benefit of visiting a state. For example, to meet the latency and the jitter requirements of URLLC, the Head-of-Line (HoL) delays of packets should lie in a small range, $\left[D_{\min }, D_{\max }\right]$. The agent will receive a positive reward only when a packet is transmitted within this range. Otherwise, $r\left(\mathbf{s}(t), \mathbf{a}^{\mathrm{s}}(t)\right)=0$. To avoid zero rewards, we can design a potential function in Fig. 14 If the HoL delay of a packet is lower than $D_{\min }$, the potential function increases with the HoL delay. In this region, the packet should not be transmitted in order to get a positive $\tilde{r}\left(\mathbf{s}(t), \mathbf{a}^{\mathbf{s}}(t)\right)$. If the HoL delay is higher than $D_{\min }$, the potential function decreases with the HoL delay. In this region, the packet should be transmitted as soon as possible, otherwise $\tilde{r}\left(\mathbf{s}(t), \mathbf{a}^{\mathbf{s}}(t)\right)$ is negative.

As proved in [268], for arbitrary potential function, reward shaping does not change the optimal policy of a reinforcement learning algorithm. With this conclusion, we have the flexibility to design any potential function according to our understanding of a problem. How to design potential functions for different problems has been widely studied in the existing literature, such as [267269]. We will evaluate the performance of using reward shaping in URLLC by providing a concrete example in Section IX

2) Multi-head Critic: The DRL introduced in Section VIII-A evaluates the long-term reward of the system with a single-head critic, where the output of the critic is a scalar that approximates the value of system when an action is taken at a state. However, a wireless network consists of multiple components, such as multiple BSs and multiple users. As shown in [270], the single-head critic is not aware of the rewards of different components of a system, and hence the agent learns slowly and the algorithm can be unstable. To handle this issue, a multi-head critic was proposed in [270] to learn a separate value function for each component of the system. The difference between single-head and multi-head 


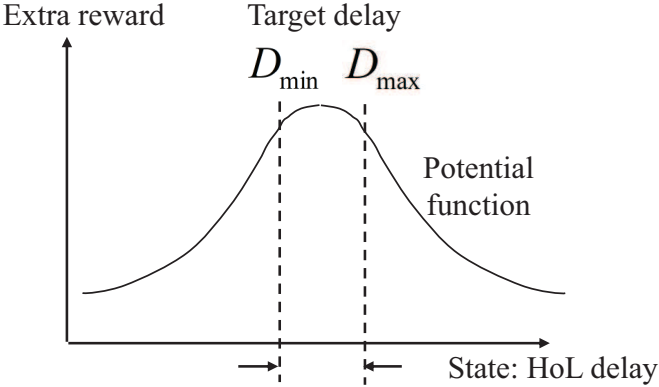

Target jitter

Fig. 14. An example of potential function [71 268].

critics is illustrated in Fig. 15, In Section IX we will apply the multi-head critic in a multi-user URLLC system to improve the learning efficiency of DRL and the reliability of each user.

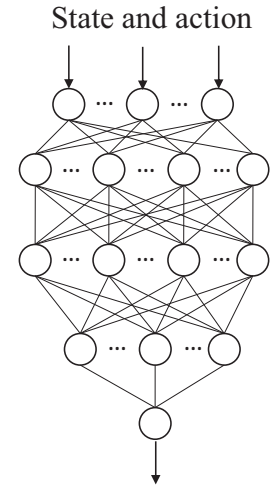

Long-term reward of the network

(a) Single-head critic

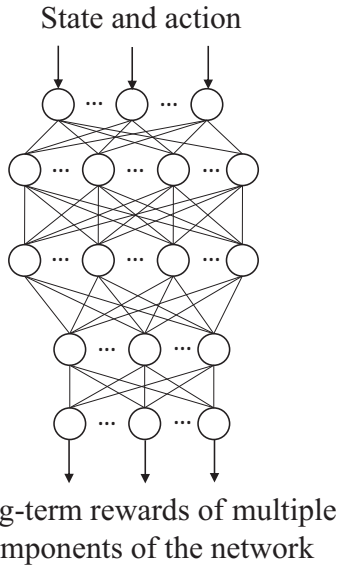

(b) Multi-head critic
Fig. 15. Difference between single-head and multi-head critics [71 270].

3) Importance Sampling: In actor-critic DRL algorithms, the state-value function is approximated by a DNN, i.e., the critic. Due to approximation errors, the action that maximizes the output of the critic may be different from the action that maximizes the true state-value function. As a result, the wireless communication system may take a wrong action leading to packet losses in URLLC. To improve the reliability of URLLC, we need to reduce the approximation errors of DRL.

In the training phase, DRL selects a batch of transitions from the replay memory to train the critic by using the Bellman equation. Let us denote the critic by $\hat{Q}\left(\mathbf{s}(t), \mathbf{a}(t) \mid \Omega_{Q}\right)$, where $\Omega_{Q}$ represents the parameters of the DNN. The approximation error at the state-action pair $(\mathbf{s}(t), \mathbf{a}(t))$ can be characterized by

$$
\left[y(t)-\hat{Q}\left(\mathbf{s}(t), \mathbf{a}^{\mathrm{s}}(t) \mid \Omega_{Q}\right)\right]^{2},
$$

where $y(t) \triangleq r(t)+\hat{Q}\left(\mathbf{s}(t+1), \mathbf{a}^{\mathbf{s}}(t+1) \mid \Omega_{Q}\right)$ is the approximation of the right-hand side of the Bellman equation in (19).

In most of the existing publications in Table VIII the transitions are selected from the replay memory with the same probability. For the state-action pairs that are rarely visited, the transitions are rarely selected in the training phase. Thus, the approximation errors could be high. To improve the approximation accuracy, importance sampling proposed in [271] is a promising approach. The basic idea is to define a weight of each transition according to the approximation error $w(t)=\left[y(t)-\hat{Q}\left(\mathbf{s}(t), \mathbf{a}^{\mathrm{s}}(t) \mid \Omega_{Q}\right)\right]^{2}$. Then, the probability that a transition will be selected is proportional to its weight. In this way, the transitions with higher approximation errors are more likely to be selected than the transitions with lower approximation errors. In Section IX] we will illustrate how does importance sampling help improve the reliability of URLLC.

\section{Constrained DRL for URLLC}

When applying DRL for the URLLC applications in Table I the long term reward is usually defined as a weighted sum of different KPIs. With different weight coefficients, the final achieved KPIs are different. To guarantee the requirements on latency and reliability, the coefficients should be optimized automatically. However, in most of the existing DRL algorithms in Table VIII these coefficients are predetermined as hyper-parameters. To determine the values of the coefficients, constrained DRL can be applied [272].

By formulating some long-term costs as statistic constraints in an MDP, we can apply constrained DRL for QoS guarantee in URLLC [273]. To illustrate how to guarantee QoS in URLLC, $M_{\mathrm{c}}$ cost functions $C_{m}^{\mathrm{MDP}}(t), m=1, \ldots, M_{\mathrm{c}}$, are considered. The cost functions are defined as follows [272],

$$
C_{m}^{\mathrm{MDP}}(t)=\mathbb{E}\left[\sum_{i=t}^{\infty} \gamma_{\mathrm{d}}^{i-t} c_{m}^{\mathrm{MDP}}\left(\mathbf{s}(i), \mathbf{a}^{\mathrm{s}}(i), \mathbf{s}(i+1)\right)\right],
$$

where $c_{m}^{\mathrm{MDP}}\left(\mathbf{s}(i), \mathbf{a}^{\mathrm{s}}(i), \mathbf{s}(i+1)\right)$ is the instantaneous cost. A long-term cost could be the average queuing delay [274,275], the access cost for handovers [276], the visitation probability of some states [277], or the cost in the tail of a risk distribution [278].

In URLLC, we aim to find the optimal policy that maximizes the long-term reward, such as resource utilization efficiency, subject to the QoS constraints, i.e.,

$$
\begin{aligned}
\max _{\pi} & \mathbb{E}[G(t)] \\
\text { s.t. } & C_{m}^{\mathrm{MDP}}(t) \leq C_{m}^{\mathrm{QoS}}, m=1, \ldots, M_{\mathrm{c}},
\end{aligned}
$$

where $C_{m}^{\mathrm{QoS}}$ is the QoS requirement. To solve problem (22), one needs to evaluate the long-term reward as well as the long-term cost. Due to this fact, finding the solution of constrained DRL is more challenging than regular DRL. To solve constrained MDP, the primal-dual method, which is referred to as the Lagrangian approach in [273], can be applied [272].

Similar to the constrained unsupervised deep learning, the Lagrangian of problem (22) can be expressed as

$$
\begin{aligned}
& L_{\mathrm{CMDP}}\left(\pi, \boldsymbol{\lambda}^{\mathrm{CMDP}}\right) \\
& =\mathbb{E}[G(t)]-\sum_{m} \lambda_{m}^{\mathrm{CMDP}}\left[C_{m}^{\mathrm{MDP}}(t)-C_{m}^{\mathrm{QoS}}\right],
\end{aligned}
$$




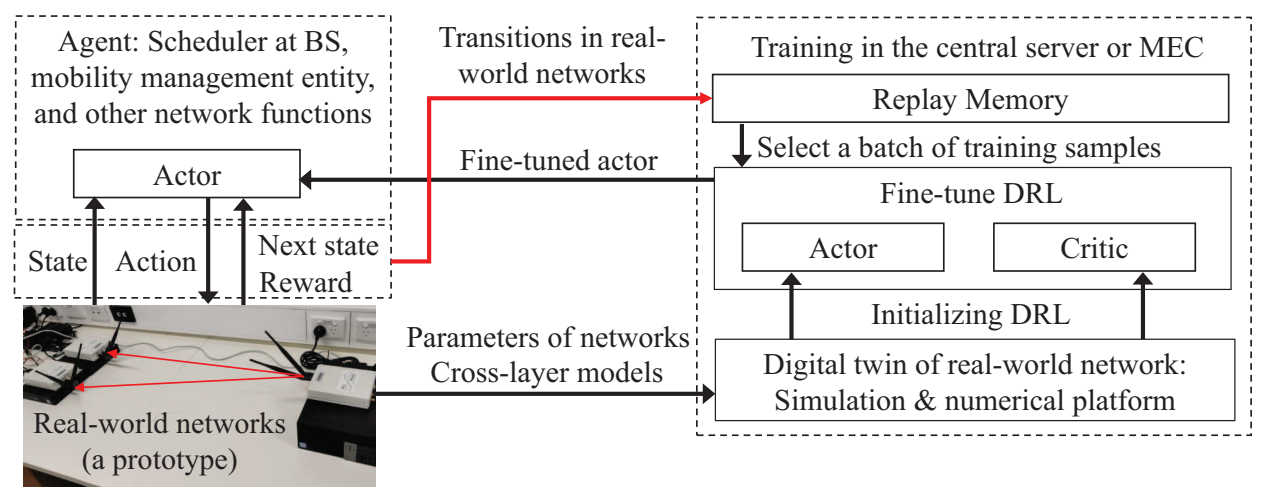

Fig. 16. A digital twin of a wireless network [10 11 11].

where $\lambda^{\mathrm{CMDP}}=\left(\lambda_{1}^{\mathrm{CMDP}}, \ldots, \lambda_{M_{\mathrm{c}}}^{\mathrm{CMDP}}\right)$ is the Lagrangian multiplier. Then, problem (22) is converted to an unconstrained problem as follows,

$$
\min _{\lambda_{m}^{\mathrm{CMDP}}>0, m=1, \ldots, M_{\mathrm{c}}} \max _{\pi} L_{\mathrm{CMDP}}\left(\pi, \boldsymbol{\lambda}^{\mathrm{CMDP}}\right) .
$$

The details of the primal-dual method can be found in [272] 273]. S. Bhatnagar et al. proved the asymptotic almost sure convergence of the method to a locally optimal solution of problem (22) [279].

Compared with the DRL algorithms in Table VIII, constrained DRL provides a more general framework for multiobjective optimization. Instead of setting some weighting coefficients among different KPIs manually, constrained DRL only includes one KPI in the objective function, while the other KPIs are considered in the constraints. By using the primaldual method, the weighting coefficients, $\lambda^{\mathrm{CMDP}}$ in (23), are optimized in the dual domain to achieve the target balance among different KPIs. For example, a weighted sum of the execution delay and the energy consumption is minimized by using DRL in [258]. If constrained DRL is applied to this problem, we can minimize the energy consumption subject to a constraint on the execution delay.

\section{E. Off-line Initialization and Online Fine-tuning of DRL in URLLC}

In this subsection, we review an architecture that enables the implementation of DRL in URLLC. The basic idea is to initialize the DRL off-line in a simulation platform and finetune the pre-trained DRL in real-world networks.

1) Digital Twins of Wireless Networks: The digital twin of a wireless network can serve as a bridge between the model-based analysis and the data-driven DRL. According to the definition in [280], "a Digital Twin is an integrated multiphysics, multiscale, probabilistic simulation of an asbuilt vehicle or system that uses the best available physical models, sensor updates, fleet history, etc., to mirror the life of its corresponding real system". It has been considered as an important approach for realizing Industrie 4.0 in [281,282].

As illustrated in Fig. 16, a central server establishes a digital twin of a real-world network with the network topology, channel and queuing models, QoS requirements, and formulas in Section IV Then, the DRL algorithm explores possible actions in the digital twin and initializes the actor and critic off-line. After off-line initialization, the actor will be sent to the control plane of the wireless network for real-time implementation.

2) Exploration in a Digital Twin: By adopting the concept of the digital twin in DRL, the agent can explore in a simulation platform to avoid potential risks in real-world systems. Given an input, the central server explores possible actions based on the output of the policy (FNN in the actor). For each action, the constraints and the utility function are evaluated in the digital twin [11]. Then, all the actions that can satisfy the constraints will be used to update the FNN in the critic to obtain a better approximation of the value function. Among these actions, the best one that maximizes the value function is saved in the memory together with the input. Finally, some training samples are randomly selected from the memory to train the FNN in the actor.

3) Transfer to Real-World Networks: Simulation environments are not exactly the same as real-world networks, which are dynamic. Thus, the actor initialized off-line may not be a good policy in non-stationary wireless networks. To handle the model mismatch problem, the system needs to update the actor and critic according to the transitions from the real-world networks. As discussed in Section VI-C2, transfer learning can be used to fine-tune pre-trained FNNs. In this way, the initial performance of the pre-trained actor is much better than the actor initialized with random parameters, and the DRL converges faster in real-world networks with transfer learning [71].

\section{F. Summary of DRL}

Domain knowledge can help establish a digital twin of a real-world network. By initializing DRL in the digital twin, the convergence time of DRL in the network can be reduced remarkably. In addition, exploration in the digital twin does not cause unexpected penalties in the network. Thus, exploration safety can be improved. To handle model mismatch, DRL keeps updating the actor and critic from the feedback of the network. In addition to the research challenges in Section VIII-B, when applying DRL in URLLC, there are two open issues.

- Unlike unsupervised deep learning that has both longterm statistic constraints and instantaneous constraints, 
constrained DRL can only guarantee long-term constraints. How to include instantaneous constraints in DRL remains unclear.

- When applying DRL in URLLC systems, the feedback of reward or penalty could be very sparse in the time horizon. This is because the packet loss probability in URLLC is extremely small. Improving the learning efficiency of DRL in URLLC systems is an urgent task.

\section{CASE Studies}

In this section, we provide concrete examples to illustrate how to guarantee the QoS requirements of URLLC by integrating domain knowledge into deep learning.

\section{A. Deep Transfer Learning for URLLC}

1) Example problem: We consider an example system in [283], where the transmit power of a BS serving multiple users is minimized by optimizing the transmit power and bandwidth allocation subject to the QoS requirements of different types of services. The problem is formulated as follows [283],

$$
\begin{aligned}
\min _{W_{k}, P_{k}^{\mathrm{t}}} & \sum_{k=1}^{K} P_{k}^{\mathrm{t}} \\
\text { s.t. } & \sum_{k=1}^{K} W_{k} \leq W_{\max }, \\
& \text { QoS constraints, } \\
& P_{k}^{\mathrm{t}} \geq 0, \text { and } W_{k} \geq 0,
\end{aligned}
$$

where $P_{k}^{\mathrm{t}}$ and $W_{k}$ are the transmit power and the bandwidth allocated to the $k$-th user, respectively, and $W_{\max }$ is the total bandwidth of the system.

The QoS constraints depend on the requirements of different kinds of services. For delay-tolerant services, the average service rate of each user should be equal to or higher than the average arrival rate of the user, i.e.,

$$
\mathbb{E}_{g_{k}}\left\{W_{k} \log _{2}\left(1+\frac{\alpha_{k} g_{k} P_{k}^{\mathrm{t}}}{W_{k} N_{0} N_{\mathrm{T}}}\right)\right\} \geq \bar{a}_{k}(t),
$$

where $N_{\mathrm{T}}$ is the number of antennas at the BS, $N_{0}$ is the single-side noise spectral density, and $\alpha_{k}$ and $g_{k}$ are the largescale and small-scale channel gains of the $k$-th user. In this system, each user is equipped with a single antenna. The instantaneous channel is available at each user, but the BS only knows $\alpha_{k}$ and the distribution of $g_{k}$.

For URLLC, the overall packet losses include decoding errors and queuing delay violations, and the E2E delay includes the transmission delay and the queuing delay. According to the conclusion in [16], we can set the required decoding error probability and the required queuing delay violation probability as equal, i.e., $\varepsilon_{\text {tot }}^{\max } / 2$. The result in [191] shows that the optimal transmission delay is one frame, $D_{\mathrm{t}}=T_{\mathrm{f}}$, and the queuing delay bound should be $D_{\mathrm{q}}^{\max }=D_{\mathrm{tot}}^{\max }-T_{\mathrm{f}}$.

To guarantee the overall packet loss probability and E2E delay, the following constraint should be satisfied,

$\mathbb{E}_{g_{k}}\left\{f_{\mathrm{Q}}\left(\sqrt{T_{\mathrm{f}} W_{k}}\left[\ln \left(1+\frac{\alpha_{k} g_{k} P_{k}^{\mathrm{t}}}{W_{k} N_{0} N_{\mathrm{T}}}\right)-\frac{u_{0} E_{B_{k}} \ln 2}{W_{k}}\right]\right)\right\}$ $\leq \varepsilon_{\text {tot }}^{\max } / 2$

where $u_{0}$ (bits/packet) is the packet size, $V \approx 1$ is applied, and the effective bandwidth of the $k$-th user, $E_{B_{k}}$ [16],

$$
E_{B_{k}}=\frac{\ln \left(2 / \varepsilon_{\text {tot }}^{\max }\right)}{\left(D_{\mathrm{tot}}^{\max }-T_{\mathrm{f}}\right) \ln \left[\frac{T_{\mathrm{f}} \ln \left(2 / \operatorname{lot}_{\mathrm{tot}}^{\max }\right)}{\lambda_{k}^{\mathrm{a}}\left(D_{\mathrm{tot}}^{\operatorname{mat}}-T_{\mathrm{f}}\right)}+1\right]}(\text { packets/s), }
$$

where $\lambda_{k}^{\mathrm{a}}$ is the average packet arrival rate of the $k$-th user.

The optimization algorithm to find labeled training samples and the supervised deep learning algorithm were developed in [283] and [12], respectively.

2) Simulation Results: The setup for simulation can be found in [283]. We use two FNNs with parameters $\Omega_{0}$ and $\Omega_{1}$ to approximate the resource allocation policies of delaytolerant services and URLLC services, respectively. For both FNNs, there are four hidden layers and each of them has 800 neurons. The dimensions of the input and output layers are 40 and 20 , respectively.

Since there is no URLLC services in the existing cellular networks, we assume that it is much easier to obtain labeled training samples for delay-tolerant services than that for URLLC services. The parameters of the first FNN, $\Omega_{0}$, are trained with the labeled samples for delay-tolerant services in set $\mathcal{S}_{0}$. To reduce the number of labeled training samples for URLLC, we initialize the values of $\Omega_{1}$ with $\Omega_{0}$ and apply network-based deep transfer learning [12]. Specifically, we fix the first three hidden layers and fine-tune the weights and bias in the last hidden layer. To evaluate the performance, we define the normalized accuracy as follows,

$$
\eta_{N N}=1-\frac{\sum_{k=1}^{K} \hat{P}_{k}^{\mathrm{t}}-\sum_{k=1}^{K} \tilde{P}_{k}^{\mathrm{t}}}{\sum_{k=1}^{K} \tilde{P}_{k}^{\mathrm{t}}},
$$

where $\hat{P}_{k}^{\mathrm{t}}$ and $\tilde{P}_{k}^{\mathrm{t}}$ are the transmit powers obtained from the supervised deep learning algorithm and the optimization algorithm, respectively.

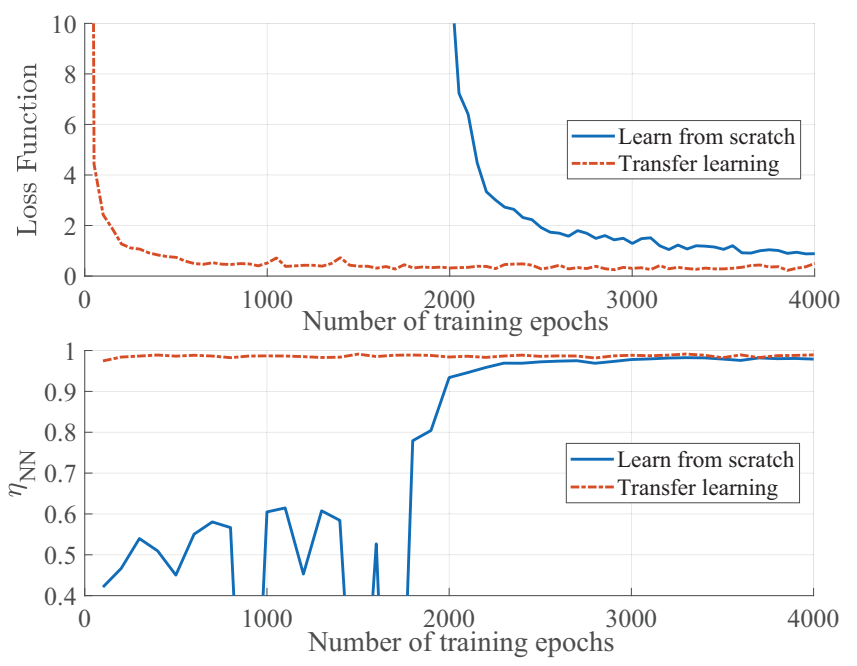

Fig. 17. Performance of deep transfer learning [12. 283]. 
As shown in Fig. 17, if there are a large number of training samples, then the normalized accuracy approaches to one as the number of training epochs increases (The optimization algorithm generates one labeled training sample in each epoch). With the network-based deep transfer learning, the normalized accuracy reaches $97 \%$ after 100 training epochs (with 100 training samples). However, if the system learns from scratch, then it takes 2500 epochs (with 2500 training samples) to achieve the same performance. Therefore, by using deep transfer learning, we can obtain a good approximation of the optimal resource allocation policy for URLLC with 100 training samples.

\section{B. Comparison of Supervised and Unsupervised Deep Learn- ing}

1) Example Problem: We take a bandwidth allocation problem in [67] to illustrate the difference between supervised and unsupervised deep learning. In this problem, the total bandwidth is minimized subject to the QoS requirement of URLLC services. The problem is formulated as follows,

$$
\begin{aligned}
\min _{W_{k}} & \sum_{k=1}^{K} W_{k} \\
\text { s.t. } & \mathbb{E}_{g_{k}}\left\{e^{-\theta_{k} s_{k}}\right\}-e^{-\theta_{k} E_{B_{k}}} \leq 0, k=1, \ldots, K, \\
& W_{k} \geq 0,
\end{aligned}
$$

where $s_{k}$ is the achievable rate in the finite blocklength regime, the value of $\theta_{k}$ is determined by the delay bound and delay violation probability according to (7), and the QoS constraints in (30) is derived from (6).

The labeled training samples for supervised deep learning are obtained from the optimization algorithm in [67]. With unsupervised deep learning, the primal-dual method is applied to solve the equivalent functional optimization problem, i.e.,

$$
\begin{aligned}
\min _{W_{k}\left(\alpha_{k}\right)} & \mathbb{E}_{\alpha_{k}}\left\{W_{k}\left(\alpha_{k}\right)\right\} \\
\text { s.t. } & \mathbb{E}_{g_{k}}\left\{e^{-\theta_{k} s_{k}\left(\alpha_{k}\right)}\right\}-e^{-\theta_{k} E_{B_{k}}} \leq 0, \\
& W_{k}\left(\alpha_{k}\right) \geq 0,
\end{aligned}
$$

where $W_{k}\left(\alpha_{k}\right)$ is a mapping from the realization of the largescale channel gain to the bandwidth allocation.

2) Simulation results: To show whether supervised and unsupervised deep learning can guarantee the QoS requirements or not, we evaluate the CCDF of relative error of the QoS constraint. Specifically, the relative error is defined as follows,

$$
\begin{aligned}
\nu & \triangleq \max \left\{\frac{\mathbb{E}_{g_{k}}\left\{e^{-\theta_{k} \hat{s}_{k}}\right\}-e^{-\theta_{k} E_{B_{k}}}}{e^{-\theta_{k} E_{B_{k}}}}, 0\right\} \\
& =\max \left\{\mathbb{E}_{g_{k}}\left\{e^{\theta_{k}\left(E_{B_{k}}-\hat{s}_{k}\right)}\right\}-1,0\right\},
\end{aligned}
$$

where $\hat{s}_{k}$ is obtained by substituting the output of the DNN, $\hat{W}_{k}$, into the expression of the achievable rate in the finite blocklength regime.

To evaluate the performance of unsupervised deep learning, the bandwidth allocation policy is trained and tested in 100 trails. In each trial, the policy is trained with 10000 iterations by using the primal-dual method and is tested with 1000 realizations of $\alpha_{k}$. The same realizations of $\alpha_{k}$ are used to train and test the bandwidth allocation policy in supervised deep learning, where the solutions obtained from the optimization algorithm, $W_{k}^{*}$, are used as the labels.

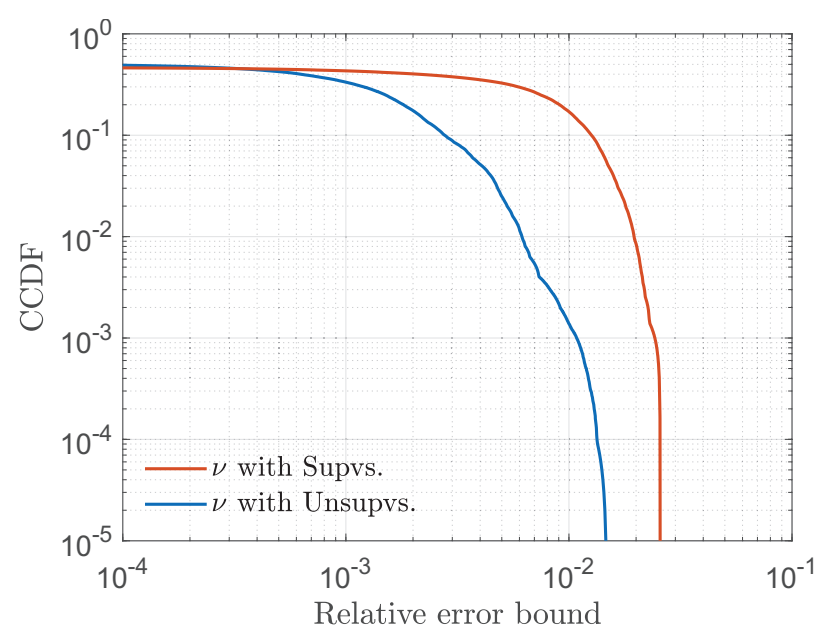

Fig. 18. Supervised deep learning versus unsupervised deep learning [67].

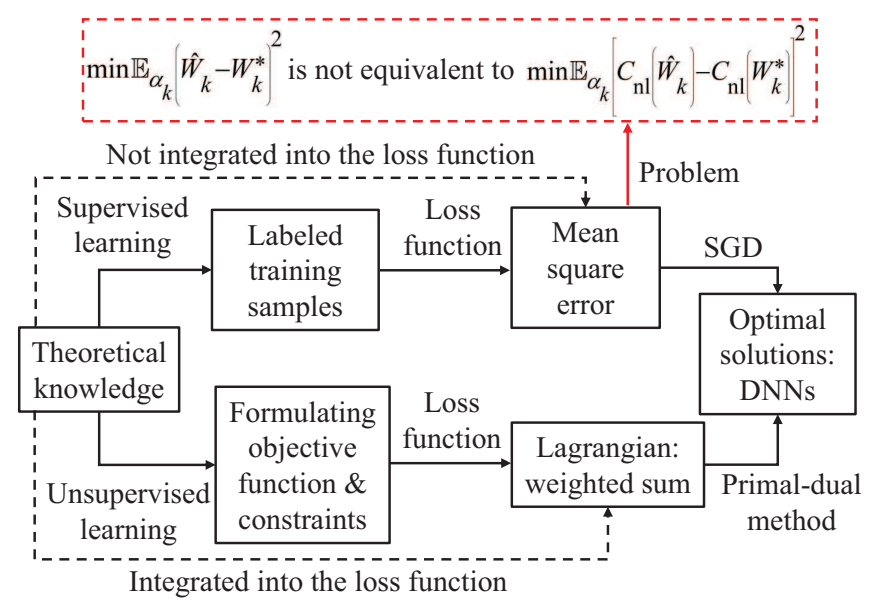

Fig. 19. Comparison of supervised and unsupervised deep learning [67].

The results in Fig. 18 shows that with probability $1-10^{-5}$, the relative errors of supervised and unsupervised deep learning are less than $3 \%$ and $2 \%$, respectively. By reserve a small portion of extra bandwidth (increasing effective capacity by $2 \sim 3 \%$ ) to each user, the deep learning approaches can guarantee the QoS requirements with a probability of $1-10^{-5}$.

The results in Fig. 18 also indicate that unsupervised deep learning outperforms supervised deep learning in terms of the approximation error of the QoS constraint. The reason is illustrated in Fig. 19, where $C_{\mathrm{nl}}$ represents the nonlinear QoS constraint in 31a). In supervised learning, the domain knowledge is used to find labeled training samples but is not integrated into the loss function, $\mathbb{E}_{\alpha_{k}}\left(\hat{W}_{k}-W_{k}^{*}\right)^{2}$. However, to minimize the approximation error of the QoS constraint, we should minimize the following loss function $\mathbb{E}_{\alpha_{k}}\left[C_{\mathrm{nl}}\left(\hat{W}_{k}\right)-C_{\mathrm{nl}}\left(W_{k}^{*}\right)\right]^{2}$. Since the QoS constraint is nonlinear, the two loss functions are not equivalent. Different from 


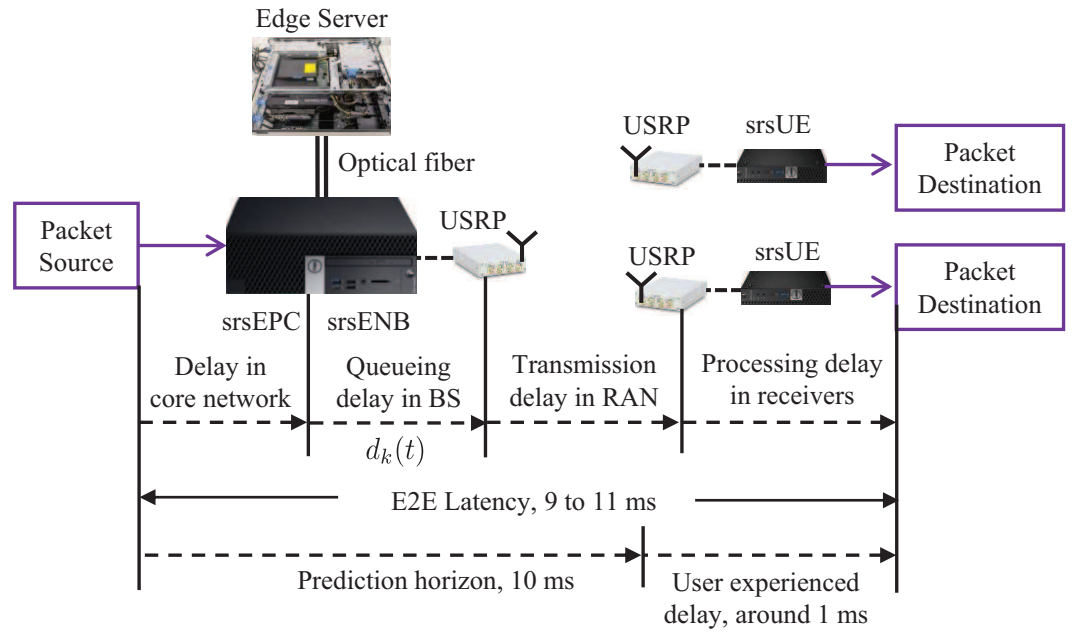

Fig. 20. Prototype for E2E delay evaluation [10,71].

supervised deep learning, the loss function of unsupervised deep learning is a weighted sum of the objective function and the QoS constraint. The policy and the weighting coefficients are optimized in the primal and dual domains, respectively. In other words, the domain knowledge is integrated into the loss function. Therefore, unsupervised deep learning outperforms supervised deep learning.

\section{Knowledge-assisted DRL}

1) Example Problem: To illustrate how to integrate domain knowledge into DRL by using the technologies in Section VIII-C, we take the scheduler design problem in [71] as an example. As shown in Fig. 21, a DL scheduler at the BS determines which users should be scheduled and how many resource blocks should be allocated to the scheduled users. Let us denote the HoL delay of the $k$-th user in the $t$-th slot as $d_{k}(t)$. To meet the latency and jitter requirements of URLLC, the users should be scheduled when $d_{k}(t)$ lies in $\left[D_{\min }, D_{\max }\right]$. As such, the jitter is $\left(D_{\max }-D_{\min }\right)$. The goal is to minimize packet losses caused by decoding errors and delay bound violations. Specifically, in the $t$-th slot, we maximize the summation of the discounted rewards of all the users,

$$
\max _{\mathbf{u}\left(\cdot \mid \Omega_{u}\right)} \mathbb{E} \sum_{i=t}^{\infty} \gamma_{\mathrm{d}}^{i-t} \sum_{k=1}^{K} r_{k}(i)
$$

where $r_{k}(i)$ is the reward of $k$-th user in the $i$-th slot, and $\mathbf{u}\left(\cdot \mid \Omega_{u}\right)$ is the actor of DRL, where $\Omega_{u}$ represents the parameters of the actor.

2) Simulation Results: We compare the performances achieved by three DRL algorithms with different definitions of state, action, and reward. The first one is a straightforward application of the deep deterministic policy gradient (DDPG) algorithm [64] (with legend "DDPG"). The state includes the HoL delays and the channel quality indicators (CQIs) of users, the action is the numbers of resource blocks allocated to users, and the reward is given by

$$
r_{k}^{[1]}(t)=\left\{\begin{array}{l}
\mathbf{1}_{D_{\min } \leq d_{k}(t) \leq D_{\max }} \cdot \mathbf{1}_{k}^{\mathrm{dec}}(t), \text { if } x_{k}(t)=1 \\
0, \text { if } x_{k}(t)=0
\end{array}\right.
$$

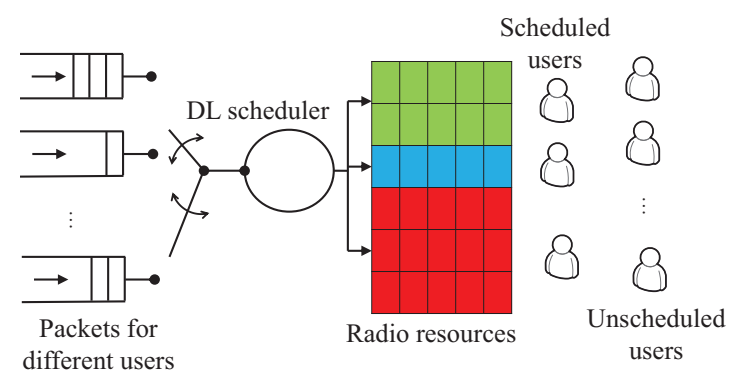

Fig. 21. DL scheduler [71].

where $\mathbf{1}_{D_{\min } \leq d_{k}(t) \leq D_{\max }}$ and $\mathbf{1}_{k}^{\mathrm{dec}}(t)$ are two indicators. If a packet is scheduled when $d_{k}(t) \in\left[D_{\min }, D_{\max }\right]$ and is successfully decoded by the receiver, then $r_{k}^{[1]}(t)=1$. Otherwise, $r_{k}^{[1]}(t)=1$.

With the second approach, DDPG is applied in a theoretical DRL framework (with legend "DDPG-T-DRL"), where the expression in (3) is applied to compute the number of resource blocks that is required to achieve a target decoding error probability, denoted by $n_{k}(t)$. Since the value of $n_{k}(t)$ is determined by the CQI of the user, the state is re-defined as the HoL delays and $n_{k}(t), k=1, \ldots, K$. Given the required numbers of resource blocks, $n_{k}(t), k=1, \ldots, K$, the scheduler only needs to determine which users should be scheduled. With the help of the expression in (3), the reward is given by

$$
r_{k}^{[2]}(t)=\left\{\begin{array}{l}
\mathbf{1}_{D_{\min } \leq d_{k}(t) \leq D_{\max }}\left(1-\epsilon_{k}^{\mathrm{c}}(t)\right), \text { if } x_{k}(t)=1 \\
0, \text { if } x_{k}(t)=0
\end{array}\right.
$$

where $\epsilon_{k}^{\mathrm{c}}(t)$ is the decoding error probability. Considering that (34) is strictly smaller than 1 , the reward can be re-defined as $\hat{r}_{k}^{[2]}(t)=-\ln \left[1-r_{k}(t)\right]$ to further improve the learning efficiency [71].

To further improve the performance, a knowledge-assisted DDPG algorithm is applied in the theoretical DRL framework in [71] (with legend "KA-DDPG-T-DRL"). The basic idea is to exploit the knowledge of the rewards of multiple users, the 
target scheduling policy, and the approximation errors of the critic. To integrating the three kinds of knowledge into DDPG, we apply the techniques discussed in Section VIII-C multihead critic, reward shaping, and importance sampling.

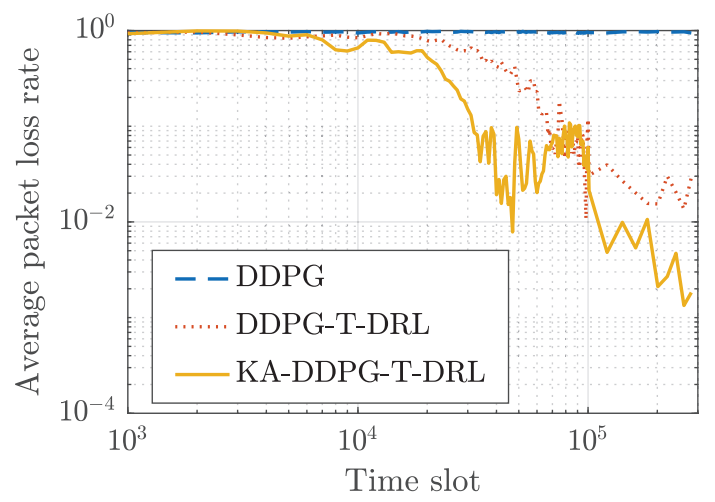

Fig. 22. Packet loss probability during the training phase, where the duration of each slot is $0.125 \mathrm{~ms}$ [71.

The details of the simulation setup of Fig. 22 can be found in [71]. To reduce the packet loss rate, we turn off the exploration after the $10^{5}$-th slot and keep fine-tuning the parameters of the actor and critic with a small learning rate, $10^{-4}$. In the first $10^{5}$ slots, the packet loss rate is evaluated every $10^{3}$ slots. After that, the packet loss rate is evaluated every $2 \times 10^{4}$ slots. The results in Fig. 22 indicate that the straightforward implementation of DDPG does not converge. With the help of the theoretical DRL framework, DDPG converges slowly and the final packet loss rate is $10^{-2}$. If the knowledge is further exploited, the algorithm converges faster and the packet loss rate can be reduced by one order of magnitude.

3) Experimental Results: To validate the architecture in Fig. 16, we built a prototype in [71]. As illustrated in Fig. 20 the prototype was built upon an open-source Long Term Evolution (LTE) software suit from Software Radio System (srs) Limited, a software company in wireless communications. The core network, the BS, and mobile devices are referred to as evolved packet core (srsEPC), eNodeB (srsENB), and user equipment (srsUE), respectively. The radio transceivers are Universal Software Radio Peripheral (USRP) B210. The E2E delay includes the delay in the core network, the queuing delay in the $\mathrm{BS}$, the transmission delay in RAN, and the processing delay in the receiver.

In this experiment, we aim to achieve $1 \mathrm{~ms}$ user experienced delay and $\pm 1 \mathrm{~ms}$ jitter in Tactile Internet, where the packet source is a tactile device [46]. In the LTE software suit, the transmission delay is longer than $1 \mathrm{~ms}$. In addition, the propagation delay in the core network is larger than $1 \mathrm{~ms}$ if the communication distance is longer than $300 \mathrm{~km}$. As a result, one can hardly achieve $1 \mathrm{~ms}$ E2E delay. To handle this issue, the prediction and communication co-design method developed in [46] is applied, where the prediction horizon is set to be $10 \mathrm{~ms}$. The transmitter predicts its future locations and sends them to the receivers $10 \mathrm{~ms}$ in advance. According to the experimental results in [10], by using an FNN to predict the trajectory in the next $10 \mathrm{~ms}$, the prediction error probability is smaller than $10^{-5}$.

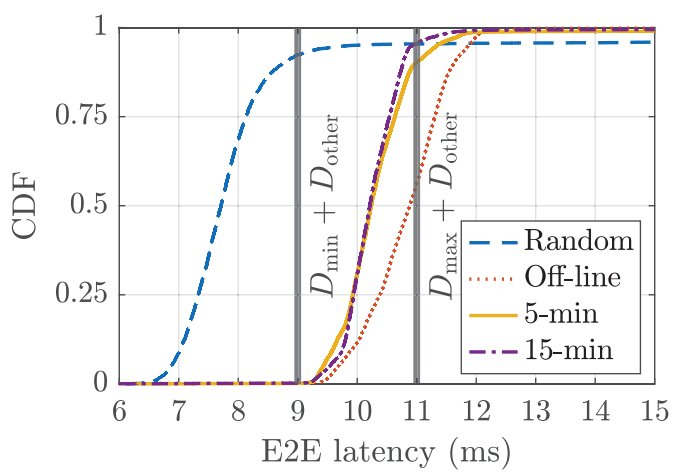

Fig. 23. Distribution of E2E delay [71.

To achieve $1 \mathrm{~ms}$ user experienced delay and $\pm 1 \mathrm{~ms}$ jitter, the E2E delay in the communication system should lie in $[9,11] \mathrm{ms}$ (given that the prediction horizon is $10 \mathrm{~ms}$ ). Since the scheduler can only control the queuing delay and cannot reduce the delay and jitter of other delay components, which cause $D_{\text {other }}=4 \mathrm{~ms}$ delay in our prototype, we set $D_{\min }=5 \mathrm{~ms}$ and $D_{\max }=7 \mathrm{~ms}$ in scheduler design. The actor and the critic are first initialized in the digital twin that mimics the behavior of the real-world network. To handle the model-mismatch problem, we fine-tune the scheduling policy by using the transitions from the measurement in the prototype.

The results in Fig. 23 shows that if we initialize the actor and the critic with random variables (with legend "Random"), the initial E2E delay does not lie in $[9,11] \mathrm{ms}$. With the initialization in the digital twin, more than half of the packets can meet the delay and jitter requirements (with legend "Offline"). After 5 minutes of fine-tuning in the prototype, around $90 \%$ packets meet the requirements (with legend "5-min"). If we keep fine-tuning the actor and the critic, the performance of the scheduler can be further improved. After 15 minutes of fine-tuning, the delay bound violation probability is around $1 \%$. Such a delay bound violation probability is still too high for URLLC. This is because the scheduler can only control the jitters in buffers of the BS, and cannot reduce the jitters of the other delay components, which become the bottleneck of the system.

To further reduce the jitter, one possible way is to do the prediction at the receivers. Since the delay and the jitter experienced by each packet can be measured by the receiver, it is possible to adjust the prediction horizon to compensate variations of the delay and the jitter at the receiver. However, the historical trajectory at the receiver is not error-free since there are some packet losses in the communication system. As a result, the prediction accuracy at the receiver will be worse than that at the transmitter. Nevertheless, the comparison of these two approaches is not available in the existing literature.

\section{Summary of Case Studies}

The examples in this section imply that the application of deep learning in URLLC is not straightforward. We need to integrate the knowledge of communications and networking 
into different kinds of learning algorithms to reduce the convergence time, guarantee the QoS constraints, and improve the final performances in terms of E2E delay, reliability, and jitter. In addition, there are some open issues should be addressed.

- The QoS of URLLC is sensitive to a lot of hyperparameters, such as the initial values of the parameters, the learning rate, and the structure of the neural network. These hyper-parameters are determined by trial-and-error in our case studies. How to find proper hyper-parameters without human efforts remains an important topic in deep learning.

- The approximation errors of continuous variables are extremely small and the QoS constraints can be satisfied by reserving a small portion of extra resources. However, when the actions are discrete variables, such as the users to be scheduled, the reliability in our prototype is still unsatisfactory for URLLC.

- To fine-tune learning algorithms in non-stationary networks, the training phase should be fast enough to follow the variations of wireless networks. According to our results, it takes around 5 to 15 minutes to fine-tune the algorithms, which is fast enough for slow varying networks. It is still very challenging to update learning algorithms in highly dynamic networks.

- In our case studies, FNNs are used to approximate the optimal policy or predict trajectories. FNNs work well in most of the small-scale problems. When the scales of the problems grow, novel structures of neural networks are needed.

\section{Future Directions}

\section{A. Extreme URLLC}

Due to the diverse QoS requirements in future application scenarios, the KPIs of URLLC, eMBB, and mMTC will be conflated in specific services. Such a new trend has been put forward in [284], and is referred to as extreme URLLC.

1) High Data Rate URLLC: As one of the killer applications in 5G networks, VR/AR applications require ultrareliable and low-latency tactile feedback and high data rate $360^{\circ}$ videos [96]. Meanwhile, as the size of devices shrinks, battery lifetime will become a bottleneck for enabling high data rate URLLC [80]. To implement VR/AR applications in future wireless networks, we need to investigate the fundamental tradeoffs among throughput, energy efficiency, reliability, and latency in communications, caching, and computing systems [35], as well as enabling technologies such as touch user interface and haptic codecs [285, 286]. With the sub- $6 \mathrm{GHz}$ spectrum, it is difficult to meet the above requirements in $5 \mathrm{G}$ cellular networks. To overcome this difficulty, Terahertz communications were applied to support VR applications in [287]. Since high-frequency communications rely on LoS paths, the blockage probability was analyzed in small cells in [287]. To further reduce the blockage probability, novel network architectures that can provide multi-path diversity are much needed.
2) Massive URLLC: Due to the explosive growth of the numbers of autonomous vehicles and mission-critical IoT devices [288], future wireless networks are expected to support massive URLLC. To support massive URLLC, novel communication and learning techniques are needed. With orthogonal multiple access technologies, the required bandwidth increases linearly with the number of devices. To achieve better tradeoffs among delay, reliability, and scalability, other multiple access technologies should be used, such as non-orthogonal multiple access and contention-based multiple access technologies [289, 290]. Meanwhile, we need to exploit the above $6 \mathrm{GHz}$ spectrum including mmWave [291] and the Terahertz band [292].

3) Secure URLLC: Future URLLC systems will suffer from different kinds of attacks that result in inefficient communications [293]. The widely used cryptography algorithms require high-complexity signal processing, and may not be suitable for URLLC, especially for IoT devices with low computing capacities. To defend against eavesdropping attacks in URLLC, physical layer security is a viable solution [49]. The maximal secret communication rate in the short blocklength regime over a wiretap channel was derived in [294]. The results show that there are tradeoffs among delay, reliability, and security. Based on this fundamental result, we can further investigate the technologies for improving physical-layer security.

\section{B. Advanced Learning Algorithms}

To meet the requirements of extreme URLLC, we need some advanced learning algorithms that were developed in recent years.

1) Multi-agent DRL with Partial Observation: MEC is a promising network architecture for high data rate/massive URLLC [90,295], where the resource allocations in communication and computing systems are intertwined. When serving VR applications in an MEC system, the system can either project a 2D field of view into a 3D field of view at the MEC or the mobile VR device [35 296]. In Industrial IoT, collecting the status of all the devices in the central server will bring considerable overheads to wireless networks, and hence the devices should be controlled in a distributed manner. To achieve this goal, multi-agent DRL with partial observation can be applied. With this framework, MEC servers, VR glasses, and IoT devices can take actions based on local observations. In this way, the overheads for exchanging control information and updating global status can be reduced remarkably [123].

2) GNNs for Flexible Network Management: Since the dimensions of the input and the output of a FNN grows with the number of devices, we need to adjust the hyper-parameters of the FNN and retrain the parameters whenever the number of devices varies. Thus, FNNs are not flexible in managing dynamic networks. To address this issue, a promising approach is to use GNNs to represent the topology of wireless networks [297]. As indicated in [298], GNN is a very general structure that can be applied to solve large-scale problems with NonEuclid data structure. Since the number of parameters of a GNN does not increase with the dimension of the input, GNNs are suitable for resource management in dynamic networks [234]. 
3) Learning with Limited Data Samples: The real-world data samples from practical systems are limited and could be non-stationary in wireless networks. To train deep learning algorithms with limited data samples, we can use GANs to generate synthetic data, such as the inter-arrival time between packets and the channel gains [125]. Another learning framework for fast adaptation is known as the few-shot learning [299]. The basic idea is to use meta learning to optimize hyperparameters, including initial parameters, learning rates, and the structures of neural networks [300,301]. After offline training in existing task, the neural networks can be transferred to new tasks with limited data samples. Essentially, by using meta learning, we are trading online learning efficiency with offline computing resources. For example, in image recognition, hundreds of graph processing unites are used to train the meta learning algorithm over several days in [302].

\section{CONCLUSION}

In this paper, we first have summarized the KPIs and challenges of URLLC in four typical application scenarios: indoor large-scale scenarios, indoor wide-area scenarios, outdoor large-scale scenarios, and outdoor wide-area scenarios. It has been argued that existing optimization algorithms cannot meet these requirements and the application of deep learning is not straightforward. To address these issues, we have developed a road-map toward URLLC based on integrating domain knowledge of communications and networking into deep learning.

Following this road-map, we have reviewed promising $6 \mathrm{G}$ network architectures and discussed how to develop deep learning frameworks based on network architectures. Then, we have revisited domain knowledge in communications and networking including analytical tools and cross-layer optimization frameworks. To show how to integrate domain knowledge into deep learning, we have introduced different kinds of learning algorithms and provided concrete examples in case studies. These results indicate that the integration outperforms straightforward applications of learning algorithms in wireless networks. Finally, we have highlighted some future directions in this area.

\section{REFERENCES}

[1] 3GPP, "Study on scenarios and requirements for next generation access technologies." TSG RAN TR38.913 R14, Jun. 2017.

[2] P. Schulz, M. Matthé, H. Klessig, et al., "Latency critical IoT applications in 5G: Perspective on the design of radio interface and network architecture," IEEE Commun. Mag., vol. 55, no. 2, pp. 70-78, Feb. 2017.

[3] O. N. C. Yilmaz, Y.-P. E. Wang, N. A. Johansson, N. Brahmi, S. A Ashraf, and J. Sachs, "Analysis of ultra-reliable and low-latency 5G communication for a factory automation use case," in IEEE International Conference on Communications (ICC) Workshop, 2015.

[4] M. Simsek, A. Aijaz, M. Dohler, et al., "5G-enabled tactile internet," IEEE J. Select. Areas Commun., vol. 34, no. 3, pp. 460-473, Mar. 2016.

[5] S. Latif, J. Qadir, S. Farooq, and M. A. Imran, "How 5G wireless (and concomitant technologies) will revolutionize healthcare?" Future Internet, vol. 9, no. 4, p. 93, 2017.

[6] K. Antonakoglou, X. Xu, E. Steinbach, T. Mahmoodi, and M. Dohler, "Towards haptic communications over the 5G tactile internet," IEEE Commun. Surveys Tuts., vol. 20, no. 4, pp. 3034-3059, 2018.

[7] GE Digital, "Industrial internet insights report." White Paper, 2015.
[8] "Germany: Industrie 4.0." Digital Transformation Monitor, 2017

[9] "Made in China 2025." Institute for Security \& Development Policy, 2017.

[10] C. She, R. Dong, Z. Gu et al., "Deep learning for ultra-reliable and low-latency communications in $6 \mathrm{G}$ networks," IEEE Netw., vol. 34 no. 5, pp. 219-225, Sep./Oct. 2020.

[11] R. Dong, C. She, W. Hardjawana, Y. Li, and B. Vucetic, "Deep learning for hybrid 5G services in mobile edge computing systems: Learn from a digital twin," IEEE Trans. Wireless Commun., no. 10, pp. 4692-4707, Oct. 2019.

[12] _ _ "Deep learning for radio resource allocation with diverse qualityof-service requirements in 5G," IEEE Trans. Wireless Commun., early access, 2020.

[13] 3GPP, "Study on new radio (NR) access technology; Physical layer aspects (release 14)." TR 38.802 V2.0.0, Stockholm, Sweden, 2017.

[14] X. Jiang, H. Shokri-Ghadikolaei, G. Fodor, E. Modiano, Z. Pang, M. Zorzi, and C. Fischione, "Low-latency networking: Where latency lurks and how to tame it," Proc. IEEE, no. 107, pp. 280-306, Feb. 2019.

[15] S. Xu, T.-H. Chang, S.-C. Lin, C. Shen, and G. Zhu, "Energy-efficient packet scheduling with finite blocklength codes: Convexity analysis and efficient algorithms," IEEE Trans. Wireless Commun., vol. 15, no. 8, pp. 5527-5540, Aug. 2016.

[16] C. She, C. Yang, and T. Q. S. Quek, "Cross-layer optimization for ultrareliable and low-latency radio access networks," IEEE Trans. Wireless Commun., vol. 17, no. 1, pp. 127-141, Jan. 2018.

[17] Y. Hu, M. Ozmen, M. C. Gursoy, and A. Schmeink, "Optimal power allocation for QoS-constrained downlink multi-user networks in the finite blocklength regime," IEEE Trans. Wireless Commun., vol. 17, no. 9, pp. 5827-5840, Sep. 2018.

[18] J. Tang, B. Shim, and T. Q. S. Quek, "Service multiplexing and revenue maximization in sliced C-RAN incorporated with URLLC and multicast eMBB," IEEE J. Sel. Areas Commun., vol. 37, no. 4, pp. 881895, Apr. 2019.

[19] C. Guo, L. Liang, and G. Y. Li, "Resource allocation for low-latency vehicular communications: An effective capacity perspective," IEEE J. Sel. Areas Commun., vol. 37, no. 4, pp. 905-917, Apr. 2019.

[20] C. Zhang, P. Patras, and H. Haddadi, "Deep learning in mobile and wireless networking: A survey," IEEE Commun. Surveys Tuts., vol. 21, no. 3, pp. 2224-2287, 2019.

[21] A. Zappone, M. Di Renzo, and M. Debbah, "Wireless networks design in the era of deep learning: Model-based, AI-based, or both?" IEEE Trans. Commun., vol. 67, no. 10, pp. 7331-7376, 2019.

[22] Z. Xiong, Y. Zhang, D. Niyato, R. Deng, P. Wang, and L.-C. Wang, "Deep reinforcement learning for mobile 5G and beyond: Fundamentals, applications, and challenges," IEEE Veh. Technol. Mag., vol. 14, no. 2, pp. 44-52, Jun. 2019.

[23] S. Gu, T. Lillicrap, I. Sutskever, and S. Levine, "Continuous deep Q-learning with model-based acceleration," in Proc. International Conference on Machine Learning (ICML), 2016.

[24] A. Goldsmith, Wireless Communications. Cambridge University Press, 2005.

[25] M. Harchol-Balter, Performance Modeling and Design of Computer Systems: Queueing Theory in Action. Cambridge University Press, 2013.

[26] C. Sun and C. Yang, "Learning to optimize with unsupervised learning: Training deep neural networks for URLLC," in Proc. IEEE Personal, Indoor and Mobile Radio Communications (PIMRC), 2019.

[27] H. He, S. Jin, C.-K. Wen, F. Gao, G. Y. Li, and Z. Xu, "Model-driven deep learning for physical layer communications," IEEE Wireless Commun., vol. 26, no. 5, pp. 77-83, 2019.

[28] M. Shirvanimoghaddam, M. Sadegh Mohamadi, R. Abbas, et al., "Short block-length codes for ultra-reliable low-latency communications," IEEE Commun. Mag., vol. 57, no. 2, pp. 130-137, Feb. 2019.

[29] P. Dymarski, S. Kula, and T. N. Huy, "QoS conditions for VoIP and VoD," J. Telecommun. and Inf. Tech., pp. 29-37, Mar. 2011.

[30] C. She, C. Yang, and T. Q. S. Quek, "Joint uplink and downlink resource configuration for ultra-reliable and low-latency communications," IEEE Trans. Commun., vol. 66, no. 5, pp. 2266-2280, May 2018.

[31] C. She, C. Liu, T. Q. Quek, C. Yang, and Y. Li, "Ultra-reliable and lowlatency communications in unmanned aerial vehicle communication systems," IEEE Trans. Commun., vol. 67, no. 5, pp. 3768-3781, May 2019.

[32] D. Ohmann, A. Awada, I. Viering, M. Simsek, and G. P. Fettweis, "Achieving high availability in wireless networks by inter-frequency 
multi-connectivity," in Proc. IEEE International Conference on Communications (ICC), 2016.

[33] 3GPP, "Service requirements for cyber-physical control applications in vertical domains," 3GPP, TS 22.104, 2018, v16.0.0.

[34] B. Holfeld, D. Wieruch, T. Wirth, L. Thiele, S. A. Ashraf, J. Huschke, I. Aktas, and J. Ansari, "Wireless communication for factory automation: An opportunity for LTE and 5G systems," IEEE Commun. Mag., vol. 54, no. 6, pp. 36-43, Jun. 2016.

[35] Y. Sun, Z. Chen, M. Tao, and H. Liu, "Communications, caching and computing for mobile virtual reality: Modeling and tradeoff," IEEE Trans. Commun., vol. 67, no. 11, pp. 7573-7586, 2019.

[36] I. Mavromatis, A. Tassi, G. Rigazzi, R. J. Piechocki, and A. Nix, "Multi-radio 5G architecture for connected and autonomous vehicles: application and design insights," arXiv preprint arXiv:1801.09510, 2018.

[37] A. Aijaz and M. Sooriyabandara, "The tactile internet for industries: A review," Proc. IEEE, vol. 107, no. 2, pp. 414-435, Feb. 2019.

[38] C. Thuemmler, C. Rolffs, A. Bollmann, G. Hindricks, and W. Buchanan, "Requirements for 5G based telemetric cardiac monitoring," in Proc. IEEE Wireless and Mobile Computing, Networking and Communications (WiMob), 2018.

[39] M. Cosovic, A. Tsitsimelis, D. Vukobratovic, J. Matamoros, and C. Anton-Haro, "5G mobile cellular networks: Enabling distributed state estimation for smart grids," IEEE Commun. Mag., vol. 55, no. 10, pp. 62-69, Oct. 2017.

[40] M. A. Lema, A. Laya, T. Mahmoodi, M. Cuevas, J. Sachs, J. Markendahl, and M. Dohler, "Business case and technology analysis for 5G low latency applications," IEEE Access, vol. 5, pp. 5917-5935, 2017.

[41] C.-F. Liu and M. Bennis, "Taming the tail of maximal information age in wireless industrial networks," IEEE Commun. Lett., vol. 23, no. 12, pp. 2442-2446, Dec. 2019.

[42] C. Kam, S. Kompella, G. D. Nguyen, J. E. Wieselthier, and A. Ephremides, "On the age of information with packet deadlines," IEEE Tran. Inf. Theory, vol. 64, no. 9, pp. 6419-6428, Sep. 2018.

[43] S. Boyd and L. Vandanberghe, Convex Optimization. Cambridge Univ. Press, 2004.

[44] D. A. Lawrence, "Stability and transparency in bilateral teleoperation," IEEE Trans. Robot. Autom., vol. 9, no. 5, pp. 624-637, Oct 1993.

[45] D. Feng, C. She, K. Ying, L. Lai, Z. Hou, T. Q. Quek, Y. Li, and B. Vucetic, "Toward ultrareliable low-latency communications: Typical scenarios, possible solutions, and open issues," IEEE Veh. Tech. Mag., vol. 14, no. 2, pp. 94-102, Jun. 2019.

[46] Z. Hou, C. She, Y. Li, Z. Li, and B. Vucetic, "Prediction and communication co-design for ultra-reliable and low-latency communications," IEEE Trans. Wirelss Commun., vol. 19, no. 2, pp. 1196-1209, Feb. 2020.

[47] T. Luettel, M. Himmelsbach, and H.-J. Wuensche, "Autonomous ground vehicles-concepts and a path to the future." Proc. IEEE, vol. 100, no. Centennial-Issue, pp. 1831-1839, May 2012.

[48] S. Kaul, M. Gruteser, V. Rai, and J. Kenney, "Minimizing age of information in vehicular networks," in Proc. IEEE Sensor and Ad Hoc Communications and Networks (SECON), 2011.

[49] R. Chen, C. Li, S. Yan, R. Malaney, and J. Yuan, "Physical layer security for ultra-reliable and low-latency communications," IEEE Wireless Commun., vol. 26, no. 5, pp. 6-11, 2019.

[50] P. Popovski, et al., "Deliverable d6.3 intermediate system evaluation results." ICT-317669-METIS/D6.3, 2014. [Online]. Available: https://www.metis2020.com/wp-content/uploads/deliverables/METIS_D6.

[51] D. Ohmann, A. Awada, I. Viering, M. Simsek, and G. P. Fettweis, "Modeling and analysis of intra-frequency multi-connectivity for high availability in 5G," in Proc. IEEE Vehicular Technology Conference (VTC) Spring, 2018.

[52] C. She, Z. Chen, C. Yang, T. Q. S. Quek, Y. Li, and B. Vucetic, "Improving network availability of ultra-reliable and low-latency communications with multi-connectivity," IEEE Trans. Commun., vol. 66, no. 11, pp. 5482-5496, Nov. 2018.

[53] J. Liu, Y. Shi, Z. M. Fadlullah, and N. Kato, "Space-air-ground integrated network: A survey," IEEE Commun. Surveys Tuts., vol. 20, no. 4, pp. 2714-2741, 2018.

[54] R. J. Kerczewski and J. H. Griner, "Control and non-payload communications links for integrated unmanned aircraft operations," NASA, 2012.

[55] L. Gupta, R. Jain, and G. Vaszkun, "Survey of important issues in UAV communication networks," IEEE Commun. Surveys Tuts., vol. 18, no. 2, pp. 1123-1152, 2016.
[56] Y. Polyanskiy, H. V. Poor, and S. Verdú, "Channel coding rate in the finite blocklength regime," IEEE Trans. Inf. Theory, vol. 56, no. 5, pp. 2307-2359, May 2010.

[57] J. Tang and X. Zhang, "Quality-of-service driven power and rate adaptation for multichannel communications over wireless links," IEEE Trans. Wireless Commun., vol. 6, no. 12, pp. 4349-4360, Dec. 2007.

[58] G. Caire, G. Taricco, and E. Biglieri, "Optimum power control over fading channels," IEEE Trans. Inf. Theory, vol. 45, no. 5, pp. 1468$1489,1999$.

[59] A. Balatsoukas-Stimming and C. Studer, "Deep unfolding for communications systems: A survey and some new directions," in Proc. IEEE Signal Processing Systems (SiPS), 2019.

[60] N. C. Luong, D. T. Hoang, S. Gong, D. Niyato, P. Wang, Y.-C. Liang, and D. I. Kim, "Applications of deep reinforcement learning in communications and networking: A survey," IEEE Commun. Surveys Tuts., vol. 21, no. 4, pp. 3133-3174, 2019.

[61] S. Dörner, S. Cammerer, J. Hoydis, and S. ten Brink, "Deep learning based communication over the air," IEEE J. Sel. Topics Signal Process., vol. 12, no. 1, pp. 132-143, Feb. 2018

[62] F. Liang, C. Shen, W. Yu, and F. Wu, "Power control for interference management via ensembling deep neural networks," in Proc. IEEE/CIC International Conference on Communications in China (ICCC), 2019.

[63] M. Eisen, C. Zhang, L. F. O. Chamon, D. D. Lee, and A. Ribeiro, "Learning optimal resource allocations in wireless systems," IEEE Trans. Signal Process., vol. 67, no. 10, pp. 2775-2790, May 2019.

[64] T. P. Lillicrap, J. J. Hunt, A. Pritzel, N. Heess, T. Erez, Y. Tassa, D. Silver, and D. Wierstra, "Continuous control with deep reinforcement learning," in Proc. International Conference on Learning Representations (ICLR), 2016.

[65] D. E. Rumelhart, G. E. Hinton, and R. J. Williams, "Learning representations by back-propagating errors," Nature, vol. 323, 1986.

[66] K. Hornik, M. Stinchcombe, and H. White, "Multilayer feedforward networks are universal approximators," Neural Networks, vol. 2, no. 5, pp. 359-366, 1989.

[67] C. Sun, C. She, and C. Yang, "Unsupervised deep learning for optimizing wireless systems with instantaneous and statistic constraints," submitted to IEEE Jounral for possible publication, 2020. [Online]. Available: https://arxiv.org/pdf/2006.01641.pdf

[68] R. S. Sutton and A. G. Barto, Reinforcement Learning: An Introduction. MIT Press, 2018.

[69] C. She, C. Yang, and T. Q. S. Quek, "Radio resource management for ultra-reliable and low-latency communications," IEEE Commun. Mag., vol. 55, no. 6, pp. 72-78, Jun. 2017.

[70] W. Yang, G. Durisi, T. Koch, and Y. Polyanskiy, "Quasi-static multipleantenna fading channels at finite blocklength," IEEE Trans. Inf. Theory, vol. 60, no. 7, pp. 4232-4264, Jul. 2014.

[71] Z. Gu, C. She, W. Hardjawana et al., "Knowledge-assisted deep reinforcement learning in 5G scheduler design: From theoretical framework to implementation," IEEE J. Sel. Areas Commun., accepted, 2021. [Online]. Available: https://arxiv.org/abs/2009.08346

[72] G. Durisi, T. Koch, and P. Popovski, "Toward massive, ultrareliable, and low-latency wireless communication with short packets," Proc. IEEE, vol. 104, no. 9, pp. 1711-1726, Aug. 2016.

[73] K. Antonakoglou, X. Xu, E. Steinbach, T. Mahmoodi, and M. Dohler, "Towards haptic communications over the 5G tactile internet," IEEE Commun. Surveys Tuts., vol. 20, no. 4, pp. 3034-3059, 2018.

[74] A. Nasrallah, A. S. Thyagaturu, Z. Alharbi, C. Wang, X. Shao, 3_v1.pdK1. Reisslein, and H. ElBakoury, "Ultra-low latency (ULL) networks: The IEEE TSN and IETF DetNet standards and related 5G ULL research," IEEE Commun. Surveys Tut., vol. 21, no. 1, pp. 88-145, 2018.

[75] K. S. Kim, D. K. Kim, C.-B. Chae, S. Choi, Y.-C. Ko, J. Kim, Y.-G. Lim, M. Yang, S. Kim, B. Lim et al., "Ultrareliable and low-latency communication techniques for tactile internet services," Proc. IEEE, vol. 107, no. 2, pp. 376-393, Feb. 2019.

[76] I. Parvez, A. Rahmati, I. Guvenc, A. I. Sarwat, and H. Dai, "A survey on low latency towards 5G: RAN, core network and caching solutions," IEEE Commun. Surveys Tuts., vol. 20, no. 4, pp. 3098-3130, 2018.

[77] M. Bennis, M. Debbah, and H. V. Poor, "Ultra-reliable and low-latency wireless communication: Tail, risk and scale," Proc. IEEE, vol. 106, no. 10 , pp. 1834-1853, 2018.

[78] G. J. Sutton, J. Zeng, R. P. Liu et al., "Enabling technologies for ultrareliable and low latency communications: From PHY and MAC layer perspectives," IEEE Commun. Surveys Tuts., vol. 21, no. 3, pp. 24882524, 2019. 
[79] R. Li, Z. Zhao, X. Zhou, G. Ding, Y. Chen, Z. Wang, and H. Zhang, "Intelligent 5G: When cellular networks meet artificial intelligence," IEEE Wireless Commun., vol. 24, no. 5, pp. 175-183, Oct. 2017.

[80] W. Saad, M. Bennis, and M. Chen, "A vision of $6 \mathrm{G}$ wireless systems: Applications, trends, technologies, and open research problems," IEEE Netw., vol. 34, no. 3, pp. 134-142, 2019.

[81] K. B. Letaief, W. Chen, Y. Shi, J. Zhang, and Y.-J. A. Zhang, "The roadmap to $6 \mathrm{G}-\mathrm{AI}$ empowered wireless networks," IEEE Commun. Mag., vol. 57, no. 8, pp. 84-90, Aug. 2019.

[82] J. Wang, C. Jiang, H. Zhang, Y. Ren, K.-C. Chen, and L. Hanzo, "Thirty years of machine learning: The road to Pareto-optimal wireless networks," IEEE Commun. Surveys Tuts., vol. 22, no. 3, pp. 1472-1514, 2020.

[83] Q. Mao, F. Hu, and Q. Hao, "Deep learning for intelligent wireless networks: A comprehensive survey," IEEE Commun. Surveys Tuts., vol. 20, no. 4, pp. 2595-2621, 2018.

[84] L. Liang, H. Ye, G. Yu, and G. Y. Li, "Deep learning based wireless resource allocation with application to vehicular networks," Proc. IEEE, vol. 108, no. 2, pp. 341-356, 2019.

[85] Y. Sun, M. Peng, Y. Zhou, Y. Huang, and S. Mao, "Application of machine learning in wireless networks: Key techniques and open issues," IEEE Commun. Surveys Tuts., vol. 21, no. 4, pp. 3072-3108, 2019.

[86] J. Park, S. Samarakoon, M. Bennis, and M. Debbah, "Wireless network intelligence at the edge," Proc. IEEE, vol. 107, no. 11, pp. 2204-2239, Nov. 2019.

[87] RP-201304, "New SID: Study on further enhancement for data collection.” 3GPP TSG RAN Meeting, Jun.-Jul. 2020. [Online]. Available: https://www.3gpp.org/ftp/tsg_ran/TSG_RAN/TSGR_88e/Docs

[88] H. Song, J. Bai, Y. Yi, J. Wu, and L. Liu, "Artificial intelligence enabled internet of things: Network architecture and spectrum access," IEEE Comput. Intell. Mag., vol. 15, no. 1, pp. 44-51, Feb. 2020.

[89] F. Tang, Y. Kawamoto, N. Kato, and J. Liu, "Future intelligent and secure vehicular network toward 6G: Machine-learning approaches," Proc. IEEE, vol. 108, no. 2, pp. 292-307, Feb. 2020.

[90] Y. Mao, C. You, J. Zhang, K. Huang, and K. B. Letaief, "A survey on mobile edge computing: The communication perspective," IEEE Commun. Surveys Tuts., vol. 19, no. 4, pp. 2322-2358, 2017.

[91] C. She, Y. Duan, G. Zhao, T. Q. S. Quek, Y. Li, and B. Vucetic, "Cross-layer design for mission-critical IoT in mobile edge computing systems," IEEE Internet of Things J., vol. 6, no. 6, pp. 9360-9374, Dec. 2019.

[92] Y. Hu and A. Schmeink, "Delay-constrained communication in edge computing networks," in Proc. IEEE Signal Processing Advances in Wireless Communications (SPAWC), 2018.

[93] C.-F. Liu, M. Bennis, and H. V. Poor, "Latency and reliability-aware task offloading and resource allocation for mobile edge computing," in IEEE Global Communications Conference (Globecom) Workshops, 2017.

[94] J. Liu and Q. Zhang, "Offloading schemes in mobile edge computing for ultra-reliable low latency communications," IEEE Access, vol. 6, pp. $12825-12837,2018$.

[95] P. Wang, Z. Zheng, B. Di, and L. Song, "HetMEC: Latency-optimal task assignment and resource allocation for heterogeneous multi-layer mobile edge computing," IEEE Trans. Wireless Commun., vol. 18, no. 10, pp. 4942-4956, Oct. 2019.

[96] M. S. Elbamby, C. Perfecto, M. Bennis, and K. Doppler, "Toward lowlatency and ultra-reliable virtual reality," IEEE Netw., vol. 32, no. 2, pp. 78-84, Mar./Apr.

[97] T. Hö $\beta$ ler, L. Scheuvens, N. Franchi, M. Simsek, and G. P. Fettweis, "Applying reliability theory for future wireless communication networks," in Proc. IEEE Personal, Indoor and Mobile Radio Communications (PIMRC), 2017.

[98] J. J. Nielsen, R. Liu, and P. Popovski, "Ultra-reliable low latency communication using interface diversity," IEEE Trans. Commun., vol. 66, no. 3, pp. 1322-1334, Mar. 2018.

[99] K.-C. Chen, T. Zhang, R. D. Gitlin, and G. Fettweis, "Ultra-low latency mobile networking," IEEE Netw., vol. 33, no. 2, pp. 181-187, Mar./Apr.

[100] J. M. Walsh, S. Weber, and C. Wa Maina, "Optimal rate-delay tradeoffs and delay mitigating codes for multipath routed and network coded networks," IEEE Trans. Inf. Theory, vol. 55, no. 12, pp. 5491-5510, Dec. 2009.

[101] X. Xu, D. Veitch, Y. Li, and B. Vucetic, "Minimum cost reconfigurable network template design with guaranteed QoS," IEEE Trans. Commun., vol. 68, no. 2, pp. 1013-1024, Feb. 2020.
[102] N. Bui, M. Cesana, S. A. Hosseini, Q. Liao, I. Malanchini, and J. Widmer, "A survey of anticipatory mobile networking: Context-based classification, prediction methodologies, and optimization techniques," IEEE Commun. Surveys Tuts., vol. 19, no. 3, pp. 1790-1821, 2017.

[103] N. Bui and J. Widmer, "Data-driven evaluation of anticipatory networking in lte networks," IEEE Trans. Mobile Comput., vol. 17, no. 10, pp. 2252-2265, Oct. 2018.

[104] S.-C. Hung, H. Hsu, S.-M. Cheng, Q. Cui, and K.-C. Chen, "Delay guaranteed network association for mobile machines in heterogeneous cloud radio access network," IEEE Trans. Mobile Comput., vol. 17, no. 12 , pp. 2744-2760, Dec. 2018.

[105] 5GPPP Architecture Working Group, "View on 5G architecture," in 5G Architecture White Paper, Dec. 2017.

[106] J. W. Won and J. M. Ahn, "3GPP URLLC patent analysis," ICT Express, 2020.

[107] A. D. Domenico, Y.-F. Liu, and W. Yu, "Optimal computational resource allocation and network slicing deployment in 5G hybrid CRAN," in Proc. IEEE International Conference on Communications ICC, 2019.

[108] N. V. Huynh, D. T. Hoang, D. N. Nguyen, and E. Dutkiewicz, "Real-time network slicing with uncertain demand: A deep learning approach," in Proc. IEEE International Conference on Communications (ICC), 2019.

[109] B. Ojaghi, F. Adelantado, E. Kartsakli, A. Antonopoulos, and C. Verikoukis, "Sliced-RAN: Joint slicing and functional split in future $5 \mathrm{G}$ radio access networks," in Proc. IEEE International Conference on Communications ICC, 2019.

[110] L. Tello-Oquendo, I. F. Akyildiz, S.-C. Lin, and V. Pla, "SDN-based architecture for providing reliable internet of things connectivity in 5G systems," in Proc. Mediterranean Ad Hoc Networking (Med-Hoc-Net), 2018.

[111] P. Popovski, K. F. Trillingsgaard, O. Simeone, and G. Durisi, "5G wireless network slicing for eMBB, URLLC, and mMTC: A communication-theoretic view," IEEE Access, vol. 6, pp. 5576555 779, 2018.

[112] B. Liu, L. Wang, M. Liu, and C. Xu, "Lifelong federated reinforcement learning: a learning architecture for navigation in cloud robotic systems," IEEE Robot. Autom. Lett., vol. 4, no. 4, pp. 4555-4562, Oct. 2019.

[113] H. B. McMahan, E. Moore, D. Ramage, S. Hampson et al., "Communication-efficient learning of deep networks from decentralized data," in Proc. International Conference on Artificial Intelligence and Statistics (AISTATS), 2017. [Online]. Available: https://arxiv.org/abs/1602.05629

[114] H. H. Yang, Z. Liu, T. Q. S. Quek, and H. V. Poor, "Scheduling policies for federated learning in wireless networks," IEEE Trans. Commun., vol. 68, no. 1, pp. 317-333, Jan. 2020.

[115] S. Samarakoon, M. Bennis, W. Saad, and M. Debbah, "Federated learning for ultra-reliable low-latency V2V communications," in Proc. IEEE Global Communications Conference (Globecom), 2018.

[116] Y. Zhao, M. Li, L. Lai, N. Suda, D. Civin, and V. Chandra, "Federated learning with non-IID data," arXiv preprint arXiv:1806.00582, 2018.

[117] L. Liu, J. Zhang, S. Song, and K. B. Letaief, "Client-edge-cloud hierarchical federated learning," in Proc. IEEE International Conference on Communications (ICC), 2020.

[118] S. J. Pan and Q. Yang, "A survey on transfer learning," IEEE Trans. Knowl. Data Eng., vol. 22, no. 10, pp. 1345-1359, Oct. 2010.

[119] C. Tan, F. Sun, T. Kong, W. Zhang, C. Yang, and C. Liu, "A survey on deep transfer learning," Proc. Springer Artificial Neural Networks and Machine Learning (ICANN), 2018.

[120] Z. Chen and B. Liu, "Lifelong machine learning," Synthesis Lectures on Artificial Intelligence and Machine Learning, vol. 12, no. 3, pp. 1-207, 2018.

[121] H. Lee, S. H. Lee, and T. Q. S. Quek, "Deep learning for distributed optimization: Applications to wireless resource management," IEEE J. Sel. Areas Commun., vol. 37, no. 10, pp. 2251-2266, Oct. 2019.

[122] L. Liang, H. Ye, and G. Y. Li, "Spectrum sharing in vehicular networks based on multi-agent reinforcement learning," IEEE J. Sel. Areas Commun., vol. 37, no. 10, pp. 2282-2292, Oct. 2019.

[123] M. K. Sharma, A. Zappone, M. Assaad, M. Debbah, and S. Vassilaras, "Distributed power control for large energy harvesting networks: A multi-agent deep reinforcement learning approach," IEEE Trans. Cog. Commun. Netw., vol. 5, no. 4, pp. 1140-1154, Dec. 2019.

[124] T. Doan, S. Maguluri, and J. Romberg, "Finite-time analysis of distributed TD (0) with linear function approximation on multi-agent reinforcement learning," in Proc. International Conference on Machine Learning (ICML), 2019. 
[125] A. T. Z. Kasgari, W. Saad, M. Mozaffari, and H. V. Poor, "Experienced deep reinforcement learning with generative adversarial networks (GANs) for model-free ultra reliable low latency communication," arXiv preprint arXiv:1911.03264, 2019.

[126] F. Ang, L. Chen, N. Zhao, Y. Chen, W. Wang, and F. R. Yu, "Robust federated learning with noisy communication," IEEE Trans. Commun., vol. 68, no. 6, pp. 3452-464, Jun. 2020.

[127] G. Zhu, Y. Wang, and K. Huang, "Broadband analog aggregation for low-latency federated edge learning," IEEE Trans. Wireless Commun., vol. 19, no. 1, pp. 491-506, Jan. 2019.

[128] C. E. Shannon, "A mathematical theory of communication," Bell Syst. Technical J., vol. 27, no. 3, pp. 379-423, 1948.

[129] J. W. Lee and R. E. Blahut, "Lower bound on BER of finite-length turbo codes based on EXIT characteristics," IEEE Commun. Lett., vol. 8, no. 4, pp. 238-240, Apr. 2004.

[130] G. Yue, B. Lu, and X. Wang, "Analysis and design of finite-length LDPC codes," IEEE Trans. Veh. Tech., vol. 56, no. 3, pp. 1321-1332, May 2007.

[131] D. Goldin and D. Burshtein, "Improved bounds on the finite length scaling of polar codes," IEEE Trans. Inf. Theory, vol. 60, no. 11, pp. 6966-6978, Nov. 2014.

[132] S. H. Hassani, K. Alishahi, and R. L. Urbanke, "Finite-length scaling for polar codes," IEEE Trans. Inf. Theory, vol. 60, no. 10, pp. 58755898, Oct. 2014

[133] K. Niu, K. Chen, J. Lin, and Q. T. Zhang, "Polar codes: Primary concepts and practical decoding algorithms," IEEE Commun. Mag, vol. 52, no. 7, pp. 192-203, Jul. 2014.

[134] P. M. Olmos and R. L. Urbanke, "A scaling law to predict the finitelength performance of spatially-coupled LDPC codes," IEEE Trans. Inf. Theory, vol. 61, no. 6, pp. 3164-3184, Jun. 2015.

[135] D. Zhang and H. Meyr, "On the performance gap between $\mathrm{ml}$ and iterative decoding of finite-length turbo-coded BICM in MIMO systems," IEEE Trans. Commun., vol. 65, no. 8, pp. 3201-3213, Aug. 2017.

[136] V. Aref, N. Rengaswamy, and L. Schmalen, "Finite-length analysis of spatially-coupled regular LDPC ensembles on burst-erasure channels," IEEE Trans. Inf. Theory, vol. 64, no. 5, pp. 3431-3449, May 2018.

[137] Y. Polyanskiy and S. Verdú, "Scalar coherent fading channel: dispersion analysis," in Proc. IEEE International Symposium on Information Theory (ISIT), 2011.

[138] A. Collins and Y. Polyanskiy, "Orthogonal designs optimize achievable dispersion for coherent miso channels," in Proc. IEEE International Symposium on Information Theory (ISIT), 2014.

[139] G. Durisi, T. Koch, J. Ostman, Y. Polyanskiy, and W. Yang, "Shortpacket communications over multiple-antenna rayleigh-fading channels," IEEE Trans. Inf. Theory, vol. 64, no. 2, pp. 618-629, Feb. 2016.

[140] Y. Polyanskiy, H. V. Poor, and S. Verdú, "Dispersion of the gilbertelliott channel," IEEE Trans. Inf. Theory, vol. 57, no. 4, pp. 1829-1848, Apr. 2011.

[141] A. Collins and Y. Polyanskiy, "Coherent multiple-antenna block-fading channels at finite blocklength," IEEE Trans. Inf. Theory, vol. 65, no. 1, pp. 380-405, Jan. 2019.

[142] A. Lancho, J. Östman, G. Durisi, T. Koch, and G. Vazquez-Vilar, "Saddlepoint approximations for short-packet wireless communications," IEEE Trans. Wireless Commun., vol. 19, no. 7, pp. 4831-4846, Jul. 2020.

[143] R. A. Berry and R. G. Gallager, "Communication over fading channels with delay constraints," IEEE Trans. Inf. Theory, vol. 48, no. 5, pp. 1135-1149, May 2002.

[144] M. J. Neely, "Optimal energy and delay tradeoffs for multiuser wireless downlinks," IEEE Trans. Inf. Theory, vol. 53, no. 9, pp. 3095-3113, Sep. 2007.

[145] Y. Li, M. Sheng, Y. Shi, X. Ma, and W. Jiao, "Energy efficiency and delay tradeoff for time-varying and interference-free wireless networks," IEEE Trans. Wireless Commun., vol. 13, no. 11, pp. 59215931, Nov. 2014.

[146] Z. Niu, X. Guo, S. Zhou, and P. R. Kumar, "Characterizing energydelay tradeoff in hyper-cellular networks with base station sleeping control," IEEE J. Sel. Areas Commun., vol. 33, no. 4, pp. 641-650, Apr. 2015.

[147] E. Uysal-Biyikoglu, B. Prabhakar, and A. E. Gamal, "Energy-efficient packet transmission over a wireless link," IEEE/ACM Trans. Networking, vol. 10, no. 4, pp. 487-499, Aug. 2002.

[148] M. A. Zafer and E. Modiano, "A calculus approach to minimum energy transmission policies with quality of service guarantees," in Proc. IEEE INFCOM, 2005, pp. 548-559.
[149] I.-H. Hou and P. R. Kumar, Packets with deadlines: A framework for real-time wireless networks. Synthesis Lectures on Communication Networks, 2013.

[150] G. Zhang, T. Q. S. Quek, A. Huang, and H. Shan, "Delay and reliability tradeoffs in heterogeneous cellular networks," IEEE Trans. Commun.

[151] C. Chang and J. A. Thomas, "Effective bandwidth in high-speed digital networks," IEEE J. Sel. Areas Commun., vol. 13, no. 6, pp. 1091-1100, Aug. 1995.

[152] D. Wu and R. Negi, "Effective capacity: A wireless link model for support of quality of service," IEEE Trans. Wireless Commun., vol. 2, no. 4, pp. 630-643, Jul. 2003.

[153] W. Cheng, X. Zhang, and H. Zhang, "Joint spectrum and power efficiencies optimization for statistical QoS provisionings over SISO/MIMO wireless networks," IEEE J. Sel. Areas Commun., vol. 31, no. 5, pp. 903-915, May 2013.

[154] L. Musavian and Q. Ni, "Effective capacity maximization with statistical delay and effective energy efficiency requirements," IEEE Trans. Wireless Commun., vol. 14, no. 7, pp. 1608-1621, Jul. 2015.

[155] H. Ren, N. Liu, C. Pan, M. Elkashlan, A. Nallanathan, X. You, and L. Hanzo, "Low-latency C-RAN: An next-generation wireless approach," IEEE Veh. Tech. Mag., vol. 13, no. 2, pp. 48-56, Jun. 2018.

[156] S. Schiessl, J. Gross, and H. Al-Zubaidy, "Delay analysis for wireless fading channels with finite blocklength channel coding," in Proc. ACM Modeling, Analysis and Simulation of Wireless and Mobile Systems (MSWiM), 2015.

[157] Q. Liu, X. Jiang, and Y. Zhou, "Per-flow end-to-end delay bounds in heterogeneous wireless networks," in Proc. IEEE International Conference on Computer Communications (INFOCOM), 2017.

[158] M. A. Zafer and E. Modiano, "A calculus approach to energy-efficient data transmission with quality-of-service constraints," IEEE/ACM Trans. Networking, vol. 17, no. 3, pp. 898-911, Jun. 2009.

[159] 3GPP TR 36.814, Further Advancements for E-UTRA Physical Layer Aspects. TSG RAN v9.0.0, Mar. 2010.

[160] R. Devassy, G. Durisi, G. C. Ferrante, O. Simeone, and E. Uysal, "Reliable transmission of short packets through queues and noisy channels under latency and peak-age violation guarantees," IEEE $J$. Sel. Areas Commun., vol. 37, no. 4, pp. 721-734, Apr. 2019.

[161] A. P. Zwart and O. J. Boxma, "Sojourn time asymptotics in the M/G/1 processor sharing queue," Queueing Systems, vol. 35, pp. 141-166, 2000.

[162] D. Gross and C. Harris, Fundamentals of Queueing Theory. Wiley, 1985.

[163] W. J. Stewart, Probability, Markov Chains, Queues, and Simulation: The Mathematical Basis of Performance Modeling. Princeton University Press, 2009.

[164] J.-Y. Le Boudec and P. Thiran, Network Calculus: A Theory of Deterministic Queuing Systems for the Internet. Springer Science \& Business Media, 2001, vol. 2050.

[165] H. Al-Zubaidy, J. Liebeherr, and A. Burchard, "Network-layer performance analysis of multihop fading channels," IEEE/ACM Trans. Netw., vol. 24, no. 1, pp. 204-217, Feb. 2016.

[166] L. Liu, P. Parag, J. Tang, W.-Y. Chen, and J.-F. Chamberland, "Resource allocation and quality of service evaluation for wireless communication systems using fluid models," IEEE Trans. Inf. Theory, vol. 53, no. 5, pp. 1767-1777, May 2007.

[167] C. She, C. Yang, and L. Liu, "Energy-efficient resource allocation for MIMO-OFDM systems serving random sources with statistical QoS requirement," IEEE Trans. Commun., vol. 63, no. 11, pp. 4125-4141, Nov. 2015.

[168] C. She and C. Yang, "Energy efficiency and delay in wireless systems: Is their relation always a tradeoff?" IEEE Trans. Wireless Commun., vol. 15 , no. 11 , pp. $7215-7228$, Nov. 2016

[169] M. Amjad, L. Musavian, and M. H. Rehmani, "Effective capacity in wireless networks: A comprehensive survey," IEEE Commun. Surveys Tuts., vol. 21, no. 4, pp. 3007-3038, 2019.

[170] F. Kelly, "Notes on effective bandwidths," Stochastic Networks: Theory and Applications, 1996.

[171] M. Ozmen and M. C. Gursoy, "Wireless throughput and energy efficiency with random arrivals and statistical queuing constraints," IEEE Trans. Inf. Theory, vol. 62, no. 3, pp. 1375-1395, Mar. 2016.

[172] J. Tang and X. Zhang, "Quality-of-service driven power and rate adaptation over wireless links," IEEE Trans. Wireless Commun., vol. 6, no. 8, pp. 3058-3068, Aug. 2007.

[173] B. Soret, M. C. Aguayo-Torres, and J. T. Entrambasaguas, "Capacity with explicit delay guarantees for generic sources over correlated Rayleigh channel," IEEE Trans. Wireless Commun., vol. 9, no. 6, pp. 1901-1911, Jun. 2010. 
[174] M. C. Gursoy, "Throughput analysis of buffer-constrained wireless systems in the finite blocklength regime," EURASIP J. Wireless Commun. Netw., vol. 2013, no. 1, Dec. 2013.

[175] Y. Hu, A. Schmeink, and J. Gross, "Blocklength-limited performance of relaying under quasi-static Rayleigh channels," IEEE Trans. Wireless Commun., vol. 15, no. 7, pp. 4548-4558, Jul. 2016.

[176] D. Öhmann, M. Simsek, and G. P. Fettweis, "Achieving high availability in wireless networks by an optimal number of Rayleighfading links," in IEEE Global Communications Conference (Globecom) Workshops, 2014.

[177] M. Serror, C. Dombrowski, K. Wehrle, and J. Gross, "Channel coding versus cooperative ARQ: Reducing outage probability in ultra-low latency wireless communications," in IEEE Global Communications Conference (Globecom) Workshops, 2015.

[178] J. J. Nielsen, R. Liu, and P. Popovski, "Ultra-reliable low latency communication (URLLC) using interface diversity," IEEE Trans. Commun., vol. 66, no. 3, pp. 1322-1334, Mar. 2018.

[179] L. D. Minkova and E. Omey, "A new markov binomial distribution," Taylor \& Francis Commun. in Statistics-Theory and Methods, vol. 43, no. 13, pp. 2674-2688, Jun. 2014.

[180] A. Al-Hourani, S. Kandeepan, and A. Jamalipour, "Modeling air-toground path loss for low altitude platforms in urban environments," in Proc. IEEE Global Communications Conference (Globecom), 2014.

[181] A. Al-Hourani, S. Kandeepan, and S. Lardner, "Optimal LAP altitude for maximum coverage," IEEE Commun. Lett., vol. 3, no. 6, pp. 569572, Dec. 2014.

[182] S. S. Szyszkowicz, H. Yanikomeroglu, and J. S. Thompson, "On the feasibility of wireless shadowing correlation models," IEEE Trans. Veh. Tech., vol. 59, no. 9, pp. 4222-4236, Nov. 2010.

[183] D. Öhmann, A. Awada, I. Viering, M. Simsek, and G. P. Fettweis, "SINR model with best server association for high availability studies of wireless networks," IEEE Wireless Commun. Lett., vol. 5, no. 1, pp. 60-63, Feb. 2016.

[184] C. She, Z. Chen, C. Yang, T. Q. S. Quek, Y. Li, and B. Vucetic, "Improving network availability of ultra-reliable and low-latency communications with multi-connectivity," IEEE Trans. Commun., vol. 66, no. 11, pp. 5482-5496, Nov. 2018.

[185] Z. Jiang, A. F. Molisch, G. Caire, and Z. Niu, "Achievable rates of FDD massive MIMO systems with spatial channel correlation," IEEE Trans. Wireless Commun., vol. 14, no. 5, pp. 2868-2882, May 2015.

[186] M. Haenggi, "The local delay in Poisson networks," IEEE Trans. Inf. Theory, vol. 59, no. 3, pp. 1788-1802, Mar. 2012.

[187] P.-Y. Kong, "Power consumption and packet delay relationship for heterogeneous wireless networks," IEEE Commun. Lett., vol. 17, no. 7, pp. 1376-1379, Jul. 2013.

[188] Y. Zhong, T. Q. S. Quek, and X. Ge, "Heterogeneous cellular networks with spatio-temporal traffic: Delay analysis and scheduling," IEEE $J$. Sel. Areas Commun., vol. 35, no. 6, pp. 1373-1386, Jun. 2017.

[189] G. Ozcan and M. C. Gursoy, "Throughput of cognitive radio systems with finite blocklength codes," IEEE J. Sel. Areas Commun., vol. 31, no. 11, pp. 2541-2554, Nov. 2013.

[190] D. Tuninetti, B. Smida, N. Devroye, and H. Seferoglu, "Scheduling on the gaussian broadcast channel with hard deadlines," in Proc. IEEE International Conference on Communications (ICC), 2018.

[191] C. She, C. Yang, and T. Q. S. Quek, "Joint uplink and downlink resource configuration for ultra-reliable and low-latency communications," IEEE Trans. Commun., vol. 66, no. 5, pp. 2266-2280, May 2018

[192] Z. Hou, C. She, Y. Li, T. Q. S. Quek, and B. Vucetic, "Burstiness aware bandwidth reservation for ultra-reliable and low-latency communications in tactile internet," IEEE J. Sel. Areas Commun., vol. 36, no. 11, pp. 2401-2410, Nov. 2018.

[193] D. Malak, M. Médard, and E. M. Yeh, "Tiny codes for guaranteeable delay," IEEE J. Sel. Areas Commun., vol. 37, no. 4, pp. 809-825, Apr. 2019.

[194] S. Schiessl, J. Gross, M. Skoglund, and G. Caire, "Delay performance of the multiuser MISO downlink under imperfect CSI and finite-length coding," IEEE J. Sel. Areas Commun., vol. 37, no. 4, pp. 765-779, Apr. 2019

[195] C. Sahin, L. Liu, E. Perrins, and L. Ma, "Delay-sensitive communications over IR-HARQ: Modulation, coding latency, and reliability," IEEE J. Sel. Areas Commun., vol. 37, no. 4, pp. 749-764, Apr. 2019.

[196] S. E. Elayoubi, P. Brown, M. Deghel, and A. Galindo-Serrano, "Radio resource allocation and retransmission schemes for URLLC over 5G networks," IEEE J. Sel. Areas Commun., vol. 37, no. 4, pp. 896-904, Apr. 2019.
[197] L. Zhou, A. Wolf, and M. Motani, "On lossy multi-connectivity: Finite blocklength performance and second-order asymptotics," IEEE J. Sel. Areas Commun., vol. 37, no. 4, pp. 735-748, Apr. 2019.

[198] M. Khoshnevisan, V. Joseph, P. Gupta, F. Meshkati, R. Prakash, and P. Tinnakornsrisuphap, "5G industrial networks with CoMP for URLLC and time sensitive network architecture," IEEE J. Sel. Areas Commun., vol. 37, no. 4, pp. 947-959, Apr. 2019.

[199] M. J. Neely, "Intelligent packet dropping for optimal energy-delay tradeoffs in wireless downlinks," IEEE Trans. Autom. Control, vol. 54 no. 3, pp. 565-579, Mar. 2009.

[200] D. Tse, Fundamentals of Wireless Communication. Cambridge University Press, 2005.

[201] C. Guo, L. Liang, and G. Y. Li, "Resource allocation for vehicular communications with low latency and high reliability," IEEE Trans. Wireless Commun., vol. 18, no. 8, pp. 3887-3902, Aug. 2019.

[202] I. Al-Anbagi, M. Erol-Kantarci, and H. T. Mouftah, "A survey on crosslayer quality-of-service approaches in WSNs for delay and reliabilityaware applications," IEEE Commun. Surveys Tuts., vol. 18, no. 1, pp. 525-552, 2016.

[203] J. Farkas, L. L. Bello, and C. Gunther, "Time-sensitive networking standards," IEEE Commun. Standards Mag., vol. 2, no. 2, pp. 20-21, Jun. 2018.

[204] A. Voulodimos, N. Doulamis, A. Doulamis, and E. Protopapadakis, "Deep learning for computer vision: A brief review," Computational Intelligence and Neuroscience, vol. 2018, 2018.

[205] D. Amodei, S. Ananthanarayanan, R. Anubhai, J. Bai, E. Battenberg, C. Case, J. Casper, B. Catanzaro, Q. Cheng, G. Chen et al., "Deep speech 2: End-to-end speech recognition in english and mandarin," in Proc. International Conference on Machine Learning (ICML), 2016.

[206] R. Collobert and J. Weston, "A unified architecture for natural language processing: Deep neural networks with multitask learning," in Proc. International Conference on Machine Learning (ICML), 2008.

[207] Z. Qin, H. Ye, G. Y. Li, and B.-H. F. Juang, "Deep learning in physical layer communications," IEEE Wireless Commun., vol. 26, no. 2, pp. 93-99, Apr. 2019.

[208] J. Guo, C.-K. Wen, S. Jin, and G. Y. Li, "Convolutional neural network based multiple-rate compressive sensing for massive MIMO CSI feedback: Design, simulation, and analysis," IEEE Trans Wireless Commun., vol. 19, no. 4, pp. 2827-2840, Apr. 2020.

[209] P. Dong, H. Zhang, G. Y. Li, N. NaderiAlizadeh, and I. S. Gaspar, "Deep CNN for wideband mmwave massive MIMO channel estimation using frequency correlation," in Proc. IEEE International Conference on Acoustics, Speech, \& Signal Processing (ICASSP), 2019.

[210] Y. Yang, D. B. Smith, and S. Seneviratne, "Deep learning channel prediction for transmit power control in wireless body area networks," in Proc. IEEE International Conference on Communications (ICC), 2019.

[211] J. D. Herath, A. Seetharam, and A. Ramesh, "A deep learning model for wireless channel quality prediction," in Proc. IEEE International Conference on Communications (ICC), 2019.

[212] J. Wang, J. Tang, Z. Xu, Y. Wang, G. Xue, X. Zhang, and D. Yang, "Spatiotemporal modeling and prediction in cellular networks: A big data enabled deep learning approach," in Proc. IEEE International Conference on Computer Communications (INFOCOM), 2017.

[213] C. Qiu, Y. Zhang, Z. Feng, P. Zhang, and S. Cui, "Spatio-temporal wireless traffic prediction with recurrent neural network," IEEE Wireless Commun. Letters., vol. 7, no. 4, pp. 554-557, Aug. 2018

[214] A. Azari, P. Papapetrou, S. Denic, and G. Peters, "User traffic prediction for proactive resource management: Learning-powered approaches," in Proc. IEEE Global Communications Conference (Globecom), 2019.

[215] C. Zhang, H. Zhang, J. Qiao, D. Yuan, and M. Zhang, "Deep transfer learning for intelligent cellular traffic prediction based on cross-domain big data," IEEE J. Sel. Areas Commun., vol. 37, no. 6, pp. 1389-1401, Jun. 2019.

[216] W. Zhang, Y. Liu, T. Liu, and C. Yang, "Trajectory prediction with recurrent neural networks for predictive resource allocation," in Proc. IEEE International Conference on Signal Processing (ICSP), 2018.

[217] H. Gebrie, H. Farooq, and A. Imran, "What machine learning predictor performs best for mobility prediction in cellular networks?" in IEEE International Conference on Communications (ICC) Workshops, 2019.

[218] K. Xiao, J. Zhao, Y. He, and S. Yu, "Trajectory prediction of UAV in smart city using recurrent neural networks," in Proc. IEEE International Conference on Communications (ICC), 2019.

[219] X. Hou, J. Zhang, M. Budagavi, and S. Dey, "Head and body motion prediction to enable mobile VR experiences with low latency," in Proc. IEEE Global Communications Conference (Globecom), 2019. 
[220] M. Lopez-Martin, B. Carro, J. Lloret, S. Egea, and A. SanchezEsguevillas, "Deep learning model for multimedia quality of experience prediction based on network flow packets," IEEE Commun. Mag., vol. 56, no. 9, pp. 110-117, Sep. 2018.

[221] G. White, A. Palade, and S. Clarke, "Forecasting QoS attributes using LSTM networks," in Proc. IEEE International Joint Conference on Neural Networks (IJCNN), 2018.

[222] H. Khedher, S. Hoteit, P. Brown et al., "Processing time evaluation and prediction in cloud-RAN," in Proc. IEEE International Conference on Communications (ICC), 2019.

[223] H. Sun, X. Chen, Q. Shi, M. Hong, X. Fu, and N. D. Sidiropoulos, "Learning to optimize: Training deep neural networks for interference management," IEEE Trans. Signal Process., vol. 66, no. 20, pp. 54385453 , Oct. 2018

[224] W. Lee, M. Kim, and D.-H. Cho, "Deep power control: Transmit power control scheme based on convolutional neural network," IEEE Commun. Lett., vol. 22, no. 6, pp. 1276-1279, Jun. 2018.

[225] J. Guo and C. Yang, "Predictive resource allocation with deep learning," in Proc. IEEE Vehicular Technology Conference (VTC) Fall, 2018.

[226] Y. LeCun, Y. Bengio, and G. Hinton, "Deep learning," Nature, vol. 521, pp. 436-444, 2015.

[227] M. Leshno, V. Y. Lin, A. Pinkus, and S. Schocken, "Multilayer feedforward networks with a nonpolynomial activation function can approximate any function," Neural Networks, vol. 6, no. 6, pp. 861867, 1993.

[228] Y. Shen, Y. Shi, J. Zhang, and K. B. Letaief, "Transfer learning for mixed-integer resource allocation problems in wireless networks," in Proc. IEEE International Conference on Communications (ICC), 2019.

[229] A. Felix, S. Cammerer, S. Dörner, J. Hoydis, and S. Ten Brink, "OFDM-autoencoder for end-to-end learning of communications systems," in IEEE Signal Processing Advances in Wireless Communications (SPAWC), 2018.

[230] N. Farsad, M. Rao, and A. Goldsmith, "Deep learning for joint sourcechannel coding of text," in Proc. IEEE International Conference on Acoustics, Speech, \& Signal Processing (ICASSP), 2018.

[231] S. Xue, Y. Ma, N. Yi, and R. Tafazolli, "Unsupervised deep learning for MU-SIMO joint transmitter and noncoherent receiver design," IEEE Wireless Commun. Lett., vol. 8, no. 1, pp. 177-180, Feb. 2019.

[232] D. S. Kalogerias, M. Eisen, G. J. Pappas, and A. Ribeiro, "Model-free learning of optimal ergodic policies in wireless systems," IEEE Trans Signal Process., vol. 68, pp. 6272-6286, Oct. 2020.

[233] D. Liu, C. Sun, C. Yang, and L. Hanzo, "Optimizing wireless systems using unsupervised and reinforced-unsupervised deep learning," IEEE Netw., vol. 34, no. 4, pp. 270-277, Jul./Aug. 2020.

[234] M. Eisen and A. R. Ribeiro, "Optimal wireless resource allocation with random edge graph neural networks," IEEE Trans. Signal Process., vol. 68, pp. 2977-2991, 2020

[235] N. P. Osmolovskii, Calculus of Variations and Optimal Control. American Mathematical Soc., 1998, vol. 180

[236] J. Gregory, Constrained Optimization in the Calculus of Variations and Optimal Control Theory. Chapman and Hall/CRC, 2018.

[237] O. C. Zienkiewicz, R. L. Taylor, P. Nithiarasu, and J. Zhu, The Finite Element Method. McGraw-hill London, 1977, vol. 3.

[238] J. F. Wendt, Computational Fluid Dynamics: AnIntroduction. Springer Science \& Business Media, 2008.

[239] P. Baldi, "Autoencoders, unsupervised learning, and deep architectures," in Proc. International Conference on Machine Learning (ICML), 2012.

[240] A. Droniou and O. Sigaud, "Gated autoencoders with tied input weights," in Proc. International Conference on Machine Learning (ICML), 2013.

[241] J. E. Prussing, Optimal Spacecraft Trajectories. Oxford Univ. Press, 2017, ch. Optimal Control Theory.

[242] C. Sun, D. Liu, and C. Yang, "Model-free unsupervised learning for optimization problems with constraints," in Proc. IEEE APCC, 2019.

[243] E. Rodrigues Gomes and R. Kowalczyk, "Dynamic analysis of multiagent Q-learning with $\varepsilon$-greedy exploration," in Proc. International Conference on Machine Learning (ICML), 2009.

[244] J. Asmuth, L. Li, M. L. Littman, A. Nouri, and D. Wingate, "A bayesian sampling approach to exploration in reinforcement learning," in Proc. UAI. AUAI Press, 2009.

[245] R. Bellman, "Dynamic programming," Science, vol. 153, no. 3731, pp. 34-37, 1966

[246] V. R. Konda and J. N. Tsitsiklis, "Actor-critic algorithms," in Proc. Neural Information Processing Systems (NIPS), 2000.
[247] V. Mnih, K. Kavukcuoglu, D. Silver, A. A. Rusu, J. Veness, M. G. Bellemare, A. Graves, M. Riedmiller, A. K. Fidjeland, G. Ostrovski et al., "Human-level control through deep reinforcement learning," Nature, vol. 518, no. 7540, p. 529, 2015.

[248] D. Zeng, L. Gu, S. Pan, J. Cai, and S. Guo, "Resource management at the network edge: A deep reinforcement learning approach," IEEE Netw., vol. 33, no. 3, pp. 26-33, May/Jun. 2019.

[249] Z. Wang, L. Li, Y. Xu, H. Tian, and S. Cui, "Handover optimization via asynchronous multi-user deep reinforcement learning," in Proc. IEEE International Conference on Communications (ICC), 2018.

[250] N. Jay, N. Rotman, B. Godfrey, M. Schapira, and A. Tamar, "A deep reinforcement learning perspective on internet congestion control," in Proc. International Conference on Machine Learning (ICML), 2019.

[251] S. De Bast, R. Torrea-Duran, A. Chiumento, S. Pollin, and H. Gagacin, "Deep reinforcement learning for dynamic network slicing in IEEE 802.11 networks," in Proc. IEEE International Conference on Computer Communications (INFOCOM), 2019.

[252] R. Atallah, C. Assi, and M. Khabbaz, "Deep reinforcement learningbased scheduling for roadside communication networks," in Proc. IEEE Modeling and Optimization in Mobile, Ad hoc, and Wireless Networks (WiOpt), 2017.

[253] Y. Wei, F. R. Yu, M. Song, and Z. Han, "User scheduling and resource allocation in HetNets with hybrid energy supply: An actor-critic reinforcement learning approach," IEEE Trans. Wireless Commun., vol. 17, no. 1, pp. 680-692, Jan. 2018.

[254] S. Chinchali, P. Hu, T. Chu, M. Sharma, M. Bansal, R. Misra, M. Pavone, and S. Katti, "Cellular network traffic scheduling with deep reinforcement learning," in Proc. AAAI Conference on Artificial Intelligence, 2018

[255] I.-S. Comşa, S. Zhang, M. E. Aydin, P. Kuonen, Y. Lu, R. Trestian, and G. Ghinea, "Towards 5G: A reinforcement learning-based scheduling solution for data traffic management," IEEE Trans. Netw. Service Manag., vol. 15, no. 4, pp. 1661-1675, Dec. 2018.

[256] H. Meng, D. Chao, Q. Guo, and X. Li, "Delay-sensitive task scheduling with deep reinforcement learning in mobile-edge computing systems," in Journal of Physics: Conference Series, vol. 1229, no. 1, 2019, pp. $1-8$.

[257] Z. Xu, Y. Wang, J. Tang, J. Wang, and M. C. Gursoy, "A deep reinforcement learning based framework for power-efficient resource allocation in cloud RANs," in Proc. IEEE International Conference on Communications (ICC), 2017.

[258] J. Li, H. Gao, T. Lv, and Y. Lu, "Deep reinforcement learning based computation offloading and resource allocation for MEC," in Proc. IEEE Wireless Communications and Networking Conference (WCNC), 2018.

[259] H. Ye and G. Y. Li, "Deep reinforcement learning for resource allocation in V2V communications," in Proc. IEEE International Conference on Communications (ICC), 2018.

[260] R. Li, Z. Zhao, Q. Sun, I. Chih-Lin, C. Yang, X. Chen, M. Zhao, and H. Zhang, "Deep reinforcement learning for resource management in network slicing," IEEE Access, vol. 6, pp. 74 429-74 441, 2018.

[261] Y. Sun, M. Peng, and S. Mao, "Deep reinforcement learning-based mode selection and resource management for green fog radio access networks," IEEE Internet of Things J., vol. 6, no. 2, pp. 1960-1971, Apr. 2018.

[262] J. Chen, S. Chen, Q. Wang, B. Cao, G. Feng, and J. Hu, "iRAF: a deep reinforcement learning approach for collaborative mobile edge computing IoT networks," IEEE Internet of Things J., vol. 6, no. 4, pp. 7011-7024, Aug. 2019.

[263] C. Qi, Y. Hua, R. Li, Z. Zhao, and H. Zhang, "Deep reinforcement learning with discrete normalized advantage functions for resource management in network slicing," IEEE Commun. Lett., vol. 23, no. 8 , pp. 1337-1341, Aug. 2019.

[264] R. Shafin, H. Chen, Y. H. Nam, S. Hur, J. Park, J. Reed, L. Liu et al., "Self-tuning sectorization: Deep reinforcement learning meets broadcast beam optimization," IEEE Trans. Wireless Commun., vol. 19, no. 6, pp. 4038-4053, Jun. 2020.

[265] K. Rusek, J. Suárez-Varela, P. Almasan, P. Barlet-Ros, and A. CabellosAparicio, "RouteNet: Leveraging graph neural networks for network modeling and optimization in SDN," IEEE J. Sel. Areas Commun., vol. 38 , no. 10 , pp. 2260-2270, Oct. 2020.

[266] P. Almasan, J. Suárez-Varela, A. Badia-Sampera, K. Rusek, P. BarletRos, and A. Cabellos-Aparicio, "Deep reinforcement learning meets graph neural networks: exploring a routing optimization use case," arXiv preprint arXiv:1910.07421, 2020.

[267] O. Marom and B. Rosman, "Belief reward shaping in reinforcement learning," in Proc. AAAI Conference on Artificial Intelligence, 2018. 
[268] A. Y. Ng, D. Harada, and S. Russell, "Policy invariance under reward transformations: Theory and application to reward shaping," in Proc. International Conference on Machine Learning (ICML), 1999.

[269] H. Zou, T. Ren, D. Yan, H. Su, and J. Zhu, "Reward shaping via metalearning," arXiv preprint arXiv:1901.09330, 2019.

[270] H. Van Seijen, M. Fatemi, J. Romoff et al., "Hybrid reward architecture for reinforcement learning," in Proc. Neural Information Processing Systems (NIPS), 2017.

[271] T. Schaul, J. Quan, I. Antonoglou, and D. Silver, "Prioritized experience replay," in Proc. International Conference on Learning Representations (ICLR), 2015.

[272] Q. Liang, F. Que, and E. Modiano, "Accelerated primal-dual policy optimization for safe reinforcement learning," in Proc. Neural Information Processing Systems (NIPS), 2017.

[273] E. Altman, Constrained Markov decision processes. CRC Press, 1999, vol. 7.

[274] D. V. Djonin and V. Krishnamurthy, " $Q$-learning algorithms for constrained markov decision processes with randomized monotone policies: Application to MIMO transmission control," IEEE Trans. Signal Process., vol. 55, no. 5, pp. 2170-2181, May 2007.

[275] M. H. Ngo and V. Krishnamurthy, "Monotonicity of constrained optimal transmission policies in correlated fading channels with ARQ," IEEE Trans. Signal Process., vol. 58, no. 1, pp. 438-451, Jan. 2010.

[276] C. Sun, E. Stevens-Navarro, and V. W. Wong, "A constrained MDPbased vertical handoff decision algorithm for $4 \mathrm{G}$ wireless networks," in Proc. IEEE International Conference on Communications (ICC), 2008.

[277] J. Marecki, M. Petrik, and D. Subramanian, "Solution methods for constrained markov decision process with continuous probability modulation," in Proc. Uncertainty in Artificial Intelligence (UAI), 2013.

[278] Y. Chow, M. Ghavamzadeh, L. Janson, and M. Pavone, "Riskconstrained reinforcement learning with percentile risk criteria," $J$. Machine Learning Research, vol. 18, no. 1, pp. 1-51, 2018.

[279] S. Bhatnagar and K. Lakshmanan, "An online actor-critic algorithm with function approximation for constrained markov decision processes," J. Optim. Theory Appl., vol. 153, no. 3, pp. 688-708, Jun. 2012.

[280] E. Glaessgen and D. Stargel, "The digital twin paradigm for future NASA and US air force vehicles," in AIAA/ASME/ASCE/AHS/ASC Struct. Dyn. Mater. Conf., 2012.

[281] T. H.-J. Uhlemann, C. Lehmann, and R. Steinhilper, "The digital twin: Realizing the cyber-physical production system for industry 4.0," Procedia CIRP, vol. 61, pp. 335-340, 2017.

[282] G. Digital, "The digital twin: Compressing time-to-value for digital industrial companies," in White Paper, 2018.

[283] C. She, R. Dong, W. Hardjawana, Y. Li, and B. Vucetic, "Optimizing resource allocation for $5 \mathrm{G}$ services with diverse quality-of-service requirements," in Proc. IEEE Global Communications Conference (Globecom), 2019.

[284] J. Park, S. Samarakoon, H. Shiri, M. K. Abdel-Aziz, T. Nishio, A. Elgabli, and M. Bennis, "Extreme URLLC: Vision, challenges, and key enablers," arXiv preprint arXiv:2001.09683, 2020.

[285] V. A. de Jesus Oliveira, L. Nedel, and A. Maciel, "Assessment of an articulatory interface for tactile intercommunication in immersive virtual environments," Elsevier Computers \& Graphics, vol. 76, pp. 18-28, Nov. 2018

[286] E. Steinbach, M. Strese, M. Eid, X. Liu, A. Bhardwaj, Q. Liu, M. AlJa' afreh, T. Mahmoodi, R. Hassen, A. El Saddik et al., "Haptic codecs for the tactile internet," Proc. IEEE, vol. 107, no. 2, pp. 447-470, Feb. 2018.

[287] C. Chaccour, M. N. Soorki, W. Saad, M. Bennis, and P. Popovski, "Can terahertz provide high-rate reliable low latency communications for wireless VR?" arXiv preprint arXiv:2005.00536, 2020.

[288] Ericsson, "Internet of Things forecast," in Ericsson Mobility Report, 2019. [Online]. Available: https://www.ericsson.com/en/mobility-report/internet-of-things-forecast

[289] M. Shirvanimoghaddam, M. Dohler, and S. J. Johnson, "Massive nonorthogonal multiple access for cellular iot: Potentials and limitations," IEEE Commun. Mag., vol. 55, no. 9, pp. 55-61, Sep. 2017.

[290] B. Singh, O. Tirkkonen, Z. Li, and M. A. Uusitalo, "Contentionbased access for ultra-reliable low latency uplink transmissions," IEEE Wireless Commun. Lett., vol. 7, no. 2, pp. 182-185, Apr. 2018.

[291] I. A. Hemadeh, K. Satyanarayana, M. El-Hajjar, and L. Hanzo, "Millimeter-wave communications: Physical channel models, design considerations, antenna constructions, and link-budget," IEEE Commun. Surveys Tuts., vol. 20, no. 2, pp. 870-913, 2018.
[292] Y. Xing and T. S. Rappaport, "Propagation measurement system and approach at $140 \mathrm{GHz}$-moving to 6G and above $100 \mathrm{GHz}$," in Proc. IEEE Global Communications Conference (Globecom), 2018.

[293] C. Li, C.-P. Li, K. Hosseini, S. B. Lee, J. Jiang, W. Chen, G. Horn, T. Ji, J. E. Smee, and J. Li, "5G-based systems design for tactile internet," Proc. IEEE, vol. 107, no. 2, pp. 307-324, 2018.

[294] W. Yang, R. F. Schaefer, and H. V. Poor, "Wiretap channels: Nonasymptotic fundamental limits," IEEE Trans Inf. Theory, vol. 65, no. 7, pp. 4069-4093, Jul. 2019.

[295] M. Aazam, K. A. Harras, and S. Zeadally, "Fog computing for 5G tactile industrial internet of things: QoE-aware resource allocation model," IEEE Trans Ind. Informat., vol. 15, no. 5, pp. 3085-3092, May 2019.

[296] S. Mangiante, G. Klas, A. Navon, Z. GuanHua, J. Ran, and M. D. Silva, "VR is on the edge: How to deliver $360^{\circ}$ videos in mobile networks," in Proc. ACM VR/AR Network, 2017.

[297] F. Gama, J. Bruna, and A. Ribeiro, "Stability properties of graph neural networks," IEEE Trans. Signal Process., vol. 68, pp. 5680-5695, Sep. 2020.

[298] Z. Wu, S. Pan, F. Chen, G. Long, C. Zhang, and S. Y. Philip, "A comprehensive survey on graph neural networks," IEEE Trans. Neural Netw. Learning Syst., vol. 32, no. 1, pp. 4-24, 2020.

[299] Q. Sun, Y. Liu, T.-S. Chua, and B. Schiele, "Meta-transfer learning for few-shot learning," in Proc. IEEE Computer Vision and Pattern Recognition (CVPR), 2019.

[300] C. Finn, P. Abbeel, and S. Levine, "Model-agnostic meta-learning for fast adaptation of deep networks," in Proc. International Conference on Machine Learning (ICML), 2017.

[301] M. Andrychowicz, M. Denil, S. Gomez, M. W. Hoffman, D. Pfau, T. Schaul, B. Shillingford, and N. De Freitas, "Learning to learn by gradient descent by gradient descent," in Proc. Neural Information Processing Systems (NIPS), 2016.

[302] B. Zoph, V. Vasudevan, J. Shlens, and Q. V. Le, "Learning transferable architectures for scalable image recognition," in Proc. IEEE Computer Vision and Pattern Recognition (CVPR), 2018. 